\title{
ELEMENTARY DERIVATIONS OF IDENTITIES FOR BILATERAL BASIC HYPERGEOMETRIC SERIES
}

\author{
MICHAEL SCHLOSSER \\ Department of Mathematics, The Ohio State University, \\ 231 West 18th Avenue, Columbus, Ohio 43210, USA \\ E-mail: mschloss@math.ohio-state.edu
}

\begin{abstract}
We give elementary derivations of several classical and some new summation and transformation formulae for bilateral basic hypergeometric series. For purpose of motivation, we review our previous simple proof ("A simple proof of Bailey's very-well-poised ${ }_{6} \psi_{6}$ summation", Proc. Amer. Math. Soc., to appear) of Bailey's very-well-poised ${ }_{6} \psi_{6}$ summation. Using a similar but different method, we now give elementary derivations of some transformations for bilateral basic hypergeometric series. In particular, these include M. Jackson's very-well-poised ${ }_{8} \psi_{8}$ transformation, a very-well-poised ${ }_{10} \psi_{10}$ transformation, by induction, Slater's general transformation for very-well-poised ${ }_{2 r} \psi_{2 r}$ series, and Slater's transformation for general ${ }_{r} \psi_{r}$ series. Finally, we derive some new transformations for bilateral basic hypergeometric series of Chu-GasperKarlsson-Minton-type.
\end{abstract}

\section{INTRODUCTION}

The classical theories of hypergeometric series (cf. 35]) and $q$-hypergeometric series (cf. [15]) consist of many known summation and transformation formulae. In fact, most identities for series involving ( $q$-)binomial coefficients can be uniformly written in terms of $(q-)$ hypergeometric series. Well-known examples are the binomial theorem, the Vandermonde summation, and their " $q$-analogues". There are numerous other summations, and also transformations for $(q$-)hypergeometric series. The $q$-hypergeometric series are usually called basic hypergeometric series, where "basic" refers to the base $q$. The theory of basic hypergeometric series, which contains hypergeometric series as special cases, arose initially in combinatorics and classical analysis, and interacts similarly with number theory, statistics, physics, and representation theory of quantum Lie algebras, see Andrews [2].

The theories of unilateral (or one-sided) hypergeometric and basic hypergeometric series have quite a rich history, dating back to, at least, Euler. Formulae for bilateral (basic) hypergeometric series were not discovered until 1907 when Dougall [13, using residue calculus, derived summations for the bilateral ${ }_{2} \mathrm{H}_{2}$ and very-well-poised ${ }_{5} H_{5}$ series. Ramanujan [18] extended the $q$-binomial theorem by finding a summation formula for the bilateral ${ }_{1} \psi_{1}$ series. Later, Bailey [7], [8] carried

Date: November 1, 2000.

2000 Mathematics Subject Classification: Primary 33D15.

Keywords and phrases: bilateral basic hypergeometric series, $q$-series, Bailey's ${ }_{6} \psi_{6}$ summation, Jackson's ${ }_{8} \psi_{8}$ transformation, ${ }_{10} \psi_{10}$ transformation, Slater's transformations for well-poised ${ }_{2 r} \psi_{2 r}$ series, Slater's transformations for general ${ }_{r} \psi_{r}$ series, Chu-Gasper-Karlsson-Minton-type transformations. 
out systematical investigations on bilateral basic hypergeometric series. Further significant contributions were made by Slater [33], [35], a student of Bailey. See [15] and [35] for an excellent survey of the above classical material.

Bailey's [7, Eq. (4.7)] very-well-poised ${ }_{6} \psi_{6}$ summation (cf. [15, Eq. (5.3.1)]) is a very powerful identity, as it stands at the top of the classical hierarchy of summation formulae for bilateral series. Some of the applications of the ${ }_{6} \psi_{6}$ summation to partitions and number theory are given in Andrews [1]. Several proofs of Bailey's ${ }_{6} \psi_{6}$ summation are already known (see, e.g., Bailey [7], Slater and Lakin [36], Andrews [1], Askey and Ismail [5], and Askey [4]) which, unfortunately, are not entirely elementary. Very recently, the author [30] found a new simple proof of the very-well-poised ${ }_{6} \psi_{6}$ summation formula, directly from three applications of Rogers' [28, p. 29, second eq.] nonterminating ${ }_{6} \phi_{5}$ summation (cf. [15, Eq. (2.7.1)]) and elementary manipulations of series.

The method we used in [30] extends that already used by M. Jackson 23, Sec. 4] in her first elementary proof of Ramanujan's ${ }_{1} \psi_{1}$ summation formula 18 ] (cf. [15, Eq. (5.2.1)]). In [30], besides of giving an elementary derivation of Bailey's verywell-poised ${ }_{6} \psi_{6}$ summation, we also gave an elementary derivation of Dougall's [13. Sec. 13] ${ }_{2} H_{2}$ summation.

In this article, we apply a similar but different method to derive several classical and some new transformations for bilateral basic hypergeometric series. In fact, here we make use of unilateral transformations and combine them with bilateral series identities to deduce more complicated bilateral series identities. After recalling some standard notation for basic hypergeometric series in Section 2, we review, for purpose of motivation, our [30] elementary proof of Bailey's very-well-poised ${ }_{6} \psi_{6}$ summation in Section 3. In Section 4, we combine Bailey's [7] summation formula for a nonterminating very-well-poised ${ }_{8} \phi_{7}$ series (cf. [15, Eq. (2.11.7)]) with Bailey's ${ }_{6} \psi_{6}$ summation. As result we obtain a transformation formula for a very-well-poised ${ }_{8} \psi_{8}$ series into a sum of two ${ }_{8} \phi_{7}$ series. This transformation is equivalent to a transformation given by M. Jackson [22, Eq. (2.2)]. In Section 5, we apply our machinery to deduce a transformation formula for a very-well-poised ${ }_{10} \psi_{10}$ series into a sum of three ${ }_{10} \phi_{9}$ series. This ${ }_{10} \psi_{10}$ transformation is given implicitly by Slater [33 and explicitly by Gasper and Rahman [15, Eq. (5.6.3)]. We go even further in Section 6, where we prove Slater's [33] general transformation for very-well-poised ${ }_{2 r} \psi_{2 r}$ series by induction. Similarly, in Section 7 , we give an elementary inductive derivation of Slater's [33] general ${ }_{r} \psi_{r}$ transformation. Instead of making use of Bailey's nonterminating ${ }_{8} \phi_{7}$ summation, we utilize the nonterminating ${ }_{3} \phi_{2}$ summation here. Finally, in Section 8, using Slater's general transformations for bilateral basic hypergeometric series, we give elementary derivations of transformations of ChuGasper-Karlsson-Minton-type which seem to be new. These identities generalize some formulae recently found by Chu [10, 11].

It is worth noting that most of the classical transformations for bilateral basic hypergeometric series are proved in the literature by specializing down from the very general transformations provided by Slater [33]. For instance, this is how the very-well-poised ${ }_{8} \psi_{8}$ and ${ }_{10} \psi_{10}$ transformations (see Equations (4.1) and (5.1)) are usually derived. Slater derives her transformations in [33] by using some general transformations for basic hypergeometric series which Sears [32] derived by manipulations of series. Slater [34] also gives shorter proofs of her transformations which use contour integrals of Barnes' type. Already Watson [37] had used such integrals to derive transformations for basic hypergeometric series of any order. In contrast, 
in this article we do not use contour integration. After finding the bilateral transformations by elementary means, we do sometimes appeal to analytic continuation to extend our results. Our course of deriving the general transformations (6.1) and (7.1) is reminiscent of (but different from) Sears' analysis in 32.

The ideas in this article should open up new avenues in the theory of multiple basic hypergeometric series. Whereas in the one-dimensional theory it is possible to specialize down from general high level identities, the extension of these to multiple basic hypergeometric series are not yet known. We expect that our technique will allow to proceed from lower level identities to systematically derive the upper level ones. In particular, we plan to apply the methods of this article and of [30] in the setting of multiple basic hypergeometric series associated to root systems, see e.g. Milne [26, Gustafson [16], v. Diejen [12], and Schlosser 29].

Finally, we wish to gratefully acknowledge the helpful comments and suggestions of George Andrews, Mourad Ismail, Christian Krattenthaler and Stephen Milne.

\section{BACKGROUND AND NOTATION}

Here we recall some standard notation for $q$-series, and basic hypergeometric series (cf. Gasper and Rahman 15]).

Let $q$ be a complex number such that $0<|q|<1$. We define the $q$-shifted factorial for all integers $k$ by

$$
(a ; q)_{\infty}:=\prod_{j=0}^{\infty}\left(1-a q^{j}\right) \quad \text { and } \quad(a ; q)_{k}:=\frac{(a ; q)_{\infty}}{\left(a q^{k} ; q\right)_{\infty}} .
$$

For brevity, we employ the usual notation

$$
\left(a_{1}, \ldots, a_{m} ; q\right)_{k} \equiv\left(a_{1} ; q\right)_{k} \ldots\left(a_{m} ; q\right)_{k}
$$

where $k$ is an integer or infinity. Further, we utilize the notations

$$
{ }_{r} \phi_{s}\left[\begin{array}{l}
a_{1}, a_{2}, \ldots, a_{r} \\
b_{1}, b_{2}, \ldots, b_{s}
\end{array} ;, z\right]:=\sum_{k=0}^{\infty} \frac{\left(a_{1}, a_{2}, \ldots, a_{r} ; q\right)_{k}}{\left(q, b_{1}, \ldots, b_{s} ; q\right)_{k}}\left((-1)^{k} q^{\left(\begin{array}{c}
k \\
2
\end{array}\right)}\right)^{1+s-r} z^{k},
$$

and

$$
{ }_{r} \psi_{s}\left[\begin{array}{c}
a_{1}, a_{2}, \ldots, a_{r} \\
b_{1}, b_{2}, \ldots, b_{s}
\end{array} ; q, z\right]:=\sum_{k=-\infty}^{\infty} \frac{\left(a_{1}, a_{2}, \ldots, a_{r} ; q\right)_{k}}{\left(b_{1}, b_{2}, \ldots, b_{s} ; q\right)_{k}}\left((-1)^{k} q^{\left(\begin{array}{c}
k \\
2
\end{array}\right)}\right)^{s-r} z^{k},
$$

for basic hypergeometric ${ }_{r} \phi_{s}$ series, and bilateral basic hypergeometric ${ }_{r} \psi_{s}$ series, respectively. See [15, p. 25 and p. 125] for the criteria of when these series terminate, or, if not, when they converge. Note that, when we are considering ${ }_{r} \phi_{s}$ series in this article, we usually have $s=r-1$, in which case the factor $\left((-1)^{k} q^{\left(\begin{array}{c}k \\ 2\end{array}\right)}\right)^{1+s-r}$ in the series is just one. A similar fact holds for the ${ }_{r} \psi_{r}$ series when $s=r$.

To shorten some of our displays, we use Sears' [32] "idem" notation. The symbol "idem $\left(a ; a_{1}, a_{2}, \ldots, a_{t}\right)$ " after an expression stands for the sum of the $t$ expressions obtained from the preceding expression by interchanging $a$ with each $a_{k}$, $k=1,2, \ldots, t$.

The theory of (classical) basic hypergeometric series consists of several summation and transformation formulae involving ${ }_{r+1} \phi_{r}$ or ${ }_{r} \psi_{r}$ series. Some of the classical summation theorems require that the parameters satisfy the additional condition of being either balanced and/or very-well-poised. An ${ }_{r+1} \phi_{r}$ basic hypergeometric series is called balanced if $b_{1} \cdots b_{r}=a_{1} \cdots a_{r+1} q$ and $z=q$. An ${ }_{r+1} \phi_{r}$ 
MICHAEL SCHLOSSER

series is well-poised if $a_{1} q=a_{2} b_{1}=\cdots=a_{r+1} b_{r}$. An ${ }_{r+1} \phi_{r}$ basic hypergeometric series is called very-well-poised if it is well-poised and if $a_{2}=-a_{3}=q \sqrt{a_{1}}$. Note that the factor

$$
\frac{1-a_{1} q^{2 k}}{1-a_{1}}
$$

appears in a very-well-poised series. The parameter $a_{1}$ is usually referred to as the special parameter of such a series. Similarly, a bilateral ${ }_{r} \psi_{r}$ basic hypergeometric series is well-poised if $a_{1} b_{1}=a_{2} b_{2} \cdots=a_{r} b_{r}$ and very-well-poised if, in addition, $a_{1}=-a_{2}=q b_{1}=-q b_{2}$.

In our computations in the following sections, we make heavily use of some elementary identities involving $q$-shifted factorials which are listed in Gasper and Rahman [15, Appendix I].

\section{BAILEY'S VERY-WELL-POISED ${ }_{6} \psi_{6}$ SUMMATION}

To motivate some of our analysis in the later sections, we review here our short and simple proof of Bailey's very-well-poised ${ }_{6} \psi_{6}$ summation:

$$
\begin{array}{r}
{ }_{6} \psi_{6}\left[\begin{array}{r}
q \sqrt{a},-q \sqrt{a}, b, c, d, e \\
\sqrt{a},-\sqrt{a}, a q / b, a q / c, a q / d, a q / e
\end{array} ;, \frac{a^{2} q}{b c d e}\right] \\
=\frac{(a q, a q / b c, a q / b d, a q / b e, a q / c d, a q / c e, a q / d e, q, q / a ; q)_{\infty}}{\left(a q / b, a q / c, a q / d, a q / e, q / b, q / c, q / d, q / e, a^{2} q / b c d e ; q\right)_{\infty}}
\end{array}
$$

provided the series either terminates, or $\left|a^{2} q / b c d e\right|<1$, for convergence.

The summation formula in (3.1) is one of the most powerful identities for bilateral basic hypergeometric series. For some applications to number theory, see Andrews [1, pp. 461-468].

To prove Bailey's ${ }_{6} \psi_{6}$ summation, we start with a suitable specialization of Rogers' ${ }_{6} \phi_{5}$ summation:

$$
{ }_{6} \phi_{5}\left[\begin{array}{c}
a, q \sqrt{a},-q \sqrt{a}, b, c, d \\
\sqrt{a},-\sqrt{a}, a q / b, a q / c, a q / d
\end{array} ; q, \frac{a q}{b c d}\right]=\frac{(a q, a q / b c, a q / b d, a q / c d ; q)_{\infty}}{(a q / b, a q / c, a q / d, a q / b c d ; q)_{\infty}},
$$

provided the series either terminates, or $|a q / b c d|<1$, for convergence. Note that (3.2) is just the special case $e \mapsto a$ of (3.1).

In (3.2), we perform the simultaneous substitutions $a \mapsto c / a, b \mapsto b / a, c \mapsto c q^{n}$ and $d \mapsto c q^{-n} / a$, and obtain

$$
\begin{aligned}
& { }_{6} \phi_{5}\left[\begin{array}{c}
c / a, q \sqrt{c / a},-q \sqrt{c / a}, b / a, c q^{n}, c q^{-n} / a \\
\sqrt{c / a},-\sqrt{c / a}, c q / b, q^{1-n} / a, q^{1+n}
\end{array} ; q, \frac{a q}{b c}\right] \\
& =\frac{\left(c q / a, q^{1-n} / b, a q^{1+n} / b, q / c ; q\right)_{\infty}}{\left(c q / b, q^{1-n} / a, q^{1+n}, a q / b c ; q\right)_{\infty}},
\end{aligned}
$$

where $|a q / b c|<1$.

Using some elementary identities for $q$-shifted factorials (see, e.g., Gasper and Rahman 15. Appendix I]) we can rewrite equation (3.3) as

$$
\begin{gathered}
\frac{(c q / b, q / a, q, a q / b c ; q)_{\infty}}{(c q / a, q / b, a q / b, q / c ; q)_{\infty}} \sum_{k=0}^{\infty} \frac{\left(1-c q^{2 k} / a\right)}{(1-c / a)} \frac{(c / a, b / a ; q)_{k}(c ; q)_{n+k}(a ; q)_{n-k}}{(q, c q / b ; q)_{k}(q ; q)_{n+k}(a q / c ; q)_{n-k}}\left(\frac{a}{b}\right)^{k} \\
=\frac{(b, c ; q)_{n}}{(a q / b, a q / c ; q)_{n}}\left(\frac{a}{b}\right)^{n}
\end{gathered}
$$


In this identity, we multiply both sides by

$$
\frac{\left(1-a q^{2 n}\right)}{(1-a)} \frac{(d, e ; q)_{n}}{(a q / d, a q / e ; q)_{n}}\left(\frac{a q}{c d e}\right)^{n}
$$

and sum over all integers $n$.

On the right side we obtain

$$
{ }_{6} \psi_{6}\left[\begin{array}{c}
q \sqrt{a},-q \sqrt{a}, b, c, d, e \\
\sqrt{a},-\sqrt{a}, a q / b, a q / c, a q / d, a q / e
\end{array} ; q, \frac{a^{2} q}{b c d e}\right] .
$$

On the left side we obtain

$$
\begin{aligned}
\frac{(c q / b, q / a, q, a q / b c ; q)_{\infty}}{(c q / a, q / b, a q / b, q / c ; q)_{\infty}} \sum_{n=-\infty}^{\infty} \frac{\left(1-a q^{2 n}\right)}{(1-a)} \frac{(d, e ; q)_{n}}{(a q / d, a q / e ; q)_{n}}\left(\frac{a q}{c d e}\right)^{n} \\
\quad \times \sum_{k=0}^{\infty} \frac{\left(1-c q^{2 k} / a\right)}{(1-c / a)} \frac{(c / a, b / a ; q)_{k}(c ; q)_{n+k}(a ; q)_{n-k}}{(q, c q / b ; q)_{k}(q ; q)_{n+k}(a q / c ; q)_{n-k}}\left(\frac{a}{b}\right)^{k} .
\end{aligned}
$$

Next, we interchange summations in (3.5) and shift the inner index $n \mapsto n-k$. (Observe that the sum over $n$ is terminated by the term $(q ; q)_{n+k}^{-1}$ from below.) We obtain, again using some elementary identities for $q$-shifted factorials,

$$
\begin{aligned}
& \frac{(c q / b, q / a, q, a q / b c ; q)_{\infty}}{(c q / a, q / b, a q / b, q / c ; q)_{\infty}} \sum_{k=0}^{\infty} \frac{\left(1-c q^{2 k} / a\right)}{(1-c / a)} \frac{(c / a, b / a ; q)_{k}}{(q, c q / b ; q)_{k}} \\
& \times \frac{\left(1-a q^{-2 k}\right)}{(1-a)} \frac{(a ; q)_{-2 k}(d, e ; q)_{-k}}{(a q / c ; q)_{-2 k}(a q / d, a q / e ; q)_{-k}}\left(\frac{c d e}{b q}\right)^{k} \\
& \times \sum_{n=0}^{\infty} \frac{\left(1-a q^{-2 k+2 n}\right)}{\left(1-a q^{-2 k}\right)} \frac{\left(a q^{-2 k}, c, d q^{-k}, e q^{-k} ; q\right)_{n}}{\left(q, a q^{1-2 k} / c, a q^{1-k} / d, a q^{1-k} / e ; q\right)_{n}}\left(\frac{a q}{c d e}\right)^{n} .
\end{aligned}
$$

Now the inner sum, provided $|a q / c d e|<1$, can be evaluated by (3.2) and we obtain

$$
\begin{aligned}
& \frac{(c q / b, q / a, q, a q / b c ; q)_{\infty}}{(c q / a, q / b, a q / b, q / c ; q)_{\infty}} \sum_{k=0}^{\infty} \frac{\left(1-c q^{2 k} / a\right)}{(1-c / a)} \frac{(c / a, b / a ; q)_{k}(a q ; q)_{-2 k}}{(q, c q / b ; q)_{k}(a q / c ; q)_{-2 k}} \\
& \quad \times \frac{(d, e ; q)_{-k}}{(a q / d, a q / e ; q)_{-k}}\left(\frac{c d e}{b q}\right)^{k} \frac{\left(a q^{1-2 k}, a q^{1-k} / c d, a q^{1-k} / c e, a q / d e ; q\right)_{\infty}}{\left(a q^{1-2 k} / c, a q^{1-k} / d, a q^{1-k} / e, a q / c d e ; q\right)_{\infty}}
\end{aligned}
$$

which can be simplified to

$$
\begin{aligned}
& \frac{(c q / b, q / a, q, a q / b c, a q, a q / c d, a q / c e, a q / d e ; q)_{\infty}}{(c q / a, q / b, a q / b, q / c, a q / c, a q / d, a q / e, a q / c d e ; q)_{\infty}} \\
& \times \sum_{k=0}^{\infty} \frac{\left(1-c q^{2 k} / a\right)}{(1-c / a)} \frac{(c / a, b / a, c d / a, c e / a ; q)_{k}}{(q, c q / b, q / d, q / e ; q)_{k}}\left(\frac{a^{2} q}{b c d e}\right)^{k} .
\end{aligned}
$$

To the last sum, provided $\left|a^{2} q / b c d e\right|<1$, we can again apply (3.2) and after some simplifications we finally obtain the right side of (3.1), as desired.

Our derivation of the ${ }_{6} \psi_{6}$ summation (3.1) is simple once the nonterminating ${ }_{6} \phi_{5}$ summation (3.2) is given. But the latter summation follows by an elementary computation from F. H. Jackson's 21] terminating ${ }_{8} \phi_{7}$ summation (cf. [15. Eq. (2.6.2)]) 


$$
\begin{array}{r}
{ }_{8} \phi_{7}\left[\begin{array}{r}
a, q \sqrt{a},-q \sqrt{a}, b, c, d, a^{2} q^{1+n} / b c d, q^{-n} \\
\left.\sqrt{a},-\sqrt{a}, a q / b, a q / c, a q / d, b c d q^{-n} / a, a q^{1+n} ; q, q\right]
\end{array}\right. \\
=\frac{(a q, a q / b c, a q / b d, a q / c d ; q)_{n}}{(a q / b, a q / c, a q / d, a q / b c d ; q)_{n}}
\end{array}
$$

as $n \rightarrow \infty$. Jackson's terminating ${ }_{8} \phi_{7}$ summation itself can be proved by various ways. An algorithmic approach uses the $q$-Zeilberger algorithm, see Koornwinder [24]. For an inductive proof, see Slater [35, Sec. 3.3.1]. For another elementary classical proof, see Gasper and Rahman [15, Sec. 2.6].

Concluding this section, we would like to add another thought, kindly initiated by an anonymous referee of [30]. It is worth comparing our proof with Askey and Ismail's [5] elegant (and now classical) proof of Bailey's ${ }_{6} \psi_{6}$ summation. Their proof uses a method in this context often referred to as "Ismail's argument" since Ismail 20] was apparently the first to apply Liouville's standard analytic continuation argument in the context of bilateral basic hypergeometric series. Askey and Ismail use Rogers' ${ }_{6} \phi_{5}$ summation once to evaluate the ${ }_{6} \psi_{6}$ series at an infinite sequence and then apply analytic continuation. Here, we evaluate the ${ }_{6} \psi_{6}$ series on a domain, and, for the full theorem, we actually also need analytic continuation. In fact, we need, in addition to $\left|a^{2} q / b c d e\right|<1$ two other inequalities on $a, b, c, d, e$, namely $|a q / b c|<1$ and $|a q / c d e|<1$, in order to apply the ${ }_{6} \phi_{5}$ summation theorem. In the end, these additional conditions can be removed. In particular, both sides of identity (3.1) are analytic in $1 / c$ around the origin. So far, we have shown the identity for $|1 / c|<\min \left(|b / a q|,|d e / a q|,\left|b d e / a^{2} q\right|\right)$. By analytic continuation, we extend the identity to be valid for $|1 / c|<\left|b d e / a^{2} q\right|$, the radius of convergence of the series.

In the following sections, our objective is to find elementary derivations of some of the classical transformations for bilateral basic hypergeometric series. The method we will use is very similar to the one used in this section with the difference that for the bilateral transformations we also utilize bilateral series identities in our derivations.

\section{M. JACKSON'S VERY-WELL-POISED ${ }_{8} \psi_{8}$ TRANSFORMATION}

M. Jackson's [22, Eq. (2.2)] transformation formula (cf. [15, Eq. (5.6.2)]) of a very-well-poised ${ }_{8} \psi_{8}$ series into a sum of two ${ }_{8} \phi_{7}$ series can be stated as follows:

$$
\begin{aligned}
& { }_{8} \psi_{8}\left[\begin{array}{c}
q \sqrt{a},-q \sqrt{a}, b, c, d, e, f, g \\
\sqrt{a},-\sqrt{a}, a q / b, a q / c, a q / d, a q / e, a q / f, a q / g
\end{array} ;, \frac{a^{3} q^{2}}{b c d e f g}\right] \\
& =\frac{(q, a q, q / a, c, c / a, b q / d, b q / e, b q / f, b q / g, a q / b d, a q / b e, a q / b f, a q / b g ; q)_{\infty}}{\left(q / b, q / d, q / e, q / f, q / g, a q / b, a q / d, a q / e, a q / f, a q / g, c / b, b c / a, b^{2} q / a ; q\right)_{\infty}} \\
& \times{ }_{8} \phi_{7}\left[\begin{array}{c}
b^{2} / a, q b / \sqrt{a},-q b / \sqrt{a}, b c / a, b d / a, b e / a, b f / a, b g / a \\
b / \sqrt{a},-b / \sqrt{a}, b q / c, b q / d, b q / e, b q / f, b q / g
\end{array} ; \frac{a^{3} q^{2}}{b c d e f g}\right] \\
& +\operatorname{idem}(b ; c)
\end{aligned}
$$

where the series either terminate, or $\mid a^{3} q^{2} /$ bcdef $g \mid<1$, for convergence. (The standard symbol "idem $(b ; c)$ " is explained in the introduction.)

M. Jackson obtained this ${ }_{8} \psi_{8}$ transformation formula by specializing a general transformation of Sears 32.

To derive the above ${ }_{8} \psi_{8}$ transformation with our elementary method, we start with a suitable specialization of Bailey's summation formula for a nonterminating 
very-well-poised ${ }_{8} \phi_{7}$ series (cf. [15, Eq. (2.11.7)]). In our subsequent computations, we further make use of Bailey's ${ }_{6} \psi_{6}$ summation (3.1), and of Bailey's ${ }_{8} \phi_{7}$ transformation in 4.6.).

For convenience, we state Bailey's nonterminating very-well-poised ${ }_{8} \phi_{7}$ summation:

$$
\begin{aligned}
& { }_{8} \phi_{7}\left[\begin{array}{c}
a, q \sqrt{a},-q \sqrt{a}, b, c, d, e, f \\
\sqrt{a},-\sqrt{a}, a q / b, a q / c, a q / d, a q / e, a q / f
\end{array} ; q, q\right] \\
& +\frac{(a q, c, d, e, f, b / a, b q / c, b q / d, b q / e, b q / f ; q)_{\infty}}{\left(a / b, a q / c, a q / d, a q / e, a q / f, b c / a, b d / a, b e / a, b f / a, b^{2} q / a ; q\right)_{\infty}} \\
& \times{ }_{8} \phi_{7}\left[\begin{array}{c}
b^{2} / a, q b / \sqrt{a},-q b / \sqrt{a}, b, b c / a, b d / a, b e / a, b f / a \\
b / \sqrt{a},-b / \sqrt{a}, b q / a, b q / c, b q / d, b q / e, b q / f
\end{array} ; q, q\right] \\
& =\frac{(a q, b / a, a q / c d, a q / c e, a q / c f, a q / d e, a q / d f, a q / e f ; q)_{\infty}}{(a q / c, a q / d, a q / e, a q / f, b c / a, b d / a, b e / a, b f / a ; q)_{\infty}},
\end{aligned}
$$

where $a^{2} q=b c d e f$. Let us briefly illustrate some of the depth of this identity. Clearly, it contains the terminating ${ }_{8} \phi_{7}$ summation (3.6) and nonterminating ${ }_{6} \phi_{5}$ summation (3.2) as special cases. A probably less known fact is that the $b \rightarrow$ 1 specialization of (4.2) yields an important theta function identity (namely Eq. (5.2)]). Of course, it is interesting to know how such a rich identity, as (4.2) is, can be actually derived. Surprisingly, it can be (not trivially) derived starting from a polynomial identity, namely Bailey's [6] very-well-poised ${ }_{10} \phi_{9}$ transformation, see the exposition in Gasper and Rahman 15, Secs. 2.10 and 2.11].

To derive M. Jackson's ${ }_{8} \psi_{8}$ transformation, we perform in (4.2) the simultaneous substitutions $a \mapsto a b q / c d e, b \mapsto a q / d e, c \mapsto a q / c e, d \mapsto b q^{n}$, and $e \mapsto b q^{-n} / a$, and obtain

$$
\begin{gathered}
{ }_{8} \phi_{7}\left[\begin{array}{c}
a b q / c d e, q \sqrt{a b q / c d e},-q \sqrt{a b q / c d e}, a q / d e, a q / c e, b q^{n}, b q^{-n} / a, a q / c d \\
\sqrt{a b q / c d e},-\sqrt{a b q / c d e}, b q / c, b q / d, a q^{2-n} / c d e, a^{2} q^{2+n} / c d e, b q / e
\end{array} ;, q\right] \\
+\frac{\left(a b q^{2} / c d e, a q / c e, b q^{n}, b q^{-n} / a, a q / c d, c / b, c q / d, a q^{2-n} / b d e, a^{2} q^{2+n} / b d e, c q / e ; q\right)_{\infty}}{\left(b / c, b q / d, a q^{2-n} / c d e, a^{2} q^{2+n} / c d e, b q / e, a q / b e, c q^{n}, c q^{-n} / a, a q / b d, a c q^{2} / b d e ; q\right)_{\infty}} \\
\times{ }_{8} \phi_{7}\left[\begin{array}{c}
a c q / b d e, q \sqrt{a c q / b d e},-q \sqrt{a c q / b d e}, a q / d e, a q / b e, c q^{n}, c q^{-n} / a, a q / b d \\
\sqrt{a c q / b d e},-\sqrt{a c q / b d e}, c q / b, c q / d, a q^{2-n} / b d e, a^{2} q^{2+n} / b d e, c q / e
\end{array}\right] \\
=\frac{\left(a b q^{2} / c d e, c / b, q^{1-n} / d, a q^{1+n} / d, b c / a, a^{2} q^{2} / b c d e, q^{1-n} / e, a q^{1+n} / e ; q\right)_{\infty}}{\left(b q / d, a q^{2-n} / c d e, a^{2} q^{2+n} / c d e, b q / e, a q / b e, c q^{n}, c q^{-n} / a, a q / b d ; q\right)_{\infty}} .
\end{gathered}
$$

Using some elementary identities for $q$-shifted factorials we can rewrite this as

$$
\begin{gathered}
\frac{\left(b q / d, a q^{2} / c d e, a^{2} q^{2} / c d e, b q / e, a q / b e, c, c / a, a q / b d ; q\right)_{\infty}}{\left(a b q^{2} / c d e, c / b, q / d, a q / d, b c / a, a^{2} q^{2} / b c d e, q / e, a q / e ; q\right)_{\infty}} \\
\times \sum_{k=0}^{\infty} \frac{\left(1-a b q^{1+2 k} / c d e\right)}{(1-a b q / c d e)} \frac{(a b q / c d e, a q / d e, a q / c e, a q / c d ; q)_{k}}{(q, b q / c, b q / d, b q / e ; q)_{k}} \\
\quad \times \frac{(b ; q)_{n+k}(c d e / a q ; q)_{n-k}}{\left(a^{2} q^{2} / c d e ; q\right)_{n+k}(a q / b ; q)_{n-k}}\left(\frac{b c d e}{a^{2} q}\right)^{k} \\
+\frac{\left(c q / d, a q^{2} / b d e, a^{2} q^{2} / b d e, c q / e, a q / c e, b, b / a, a q / c d ; q\right)_{\infty}}{\left(a c q^{2} / b d e, b / c, q / d, a q / d, b c / a, a^{2} q^{2} / b c d e, q / e, a q / e ; q\right)_{\infty}}
\end{gathered}
$$




$$
\begin{gathered}
\times \sum_{k=0}^{\infty} \frac{\left(1-a c q^{1+2 k} / b d e\right)}{(1-a c q / b d e)} \frac{(a c q / b d e, a q / d e, a q / b e, a q / b d ; q)_{k}}{(q, c q / b, c q / d, c q / e ; q)_{k}} \\
\times \frac{(c ; q)_{n+k}(b d e / a q ; q)_{n-k}}{\left(a^{2} q^{2} / b d e ; q\right)_{n+k}(a q / c ; q)_{n-k}}\left(\frac{b c d e}{a^{2} q}\right)^{k} \\
=\frac{(b, c, d, e ; q)_{n}}{(a q / b, a q / c, a q / d, a q / e ; q)_{n}}
\end{gathered}
$$

Observe that on the left side of this identity the second term equals the first term where $b$ and $c$ are interchanged. This observation helps us to reduce the amount of our subsequent computations.

In identity (4.3), we multiply both sides by

$$
\frac{\left(1-a q^{2 n}\right)}{(1-a)} \frac{(f, g ; q)_{n}}{(a q / f, a q / g ; q)_{n}}\left(\frac{a^{3} q^{2}}{b c d e f g}\right)^{n}
$$

and sum over all integers $n$.

On the right side we obtain

$$
{ }_{8} \psi_{8}\left[\begin{array}{c}
q \sqrt{a},-q \sqrt{a}, b, c, d, e, f, g \\
\sqrt{a},-\sqrt{a}, a q / b, a q / c, a q / d, a q / e, a q / f, a q / g
\end{array} ;, \frac{a^{3} q^{2}}{b c d e f g}\right] .
$$

On the left side we obtain

$$
\begin{aligned}
& \frac{\left(b q / d, a q^{2} / c d e, a^{2} q^{2} / c d e, b q / e, a q / b e, c, c / a, a q / b d ; q\right)_{\infty}}{\left(a b q^{2} / c d e, c / b, q / d, a q / d, b c / a, a^{2} q^{2} / b c d e, q / e, a q / e ; q\right)_{\infty}} \\
& \times \sum_{n=-\infty}^{\infty} \frac{\left(1-a q^{2 n}\right)}{(1-a)} \frac{(f, g ; q)_{n}}{(a g / f, a q / g ; q)_{n}}\left(\frac{a^{3} q^{2}}{b c d e f g}\right)^{n} \\
& \times \sum_{k=0}^{\infty} \frac{\left(1-a b q^{1+2 k} / c d e\right)}{(1-a b q / c d e)} \frac{(a b q / c d e, a q / d e, a q / c e, a q / c d ; q)_{k}}{(q, b q / c, b q / d, b q / e ; q)_{k}} \\
& \times \frac{(b ; q)_{n+k}(c d e / a q ; q)_{n-k}}{\left(a^{2} q^{2} / c d e ; q\right)_{n+k}(a q / b ; q)_{n-k}}\left(\frac{b c d e}{a^{2} q}\right)^{k}+\operatorname{idem}(b ; c) .
\end{aligned}
$$

Next, we interchange summations in (4.4) and obtain, again using some elementary identities for $q$-shifted factorials,

$$
\begin{aligned}
& \frac{\left(b q / d, a q^{2} / c d e, a^{2} q^{2} / c d e, b q / e, a q / b e, c, c / a, a q / b d ; q\right)_{\infty}}{\left(a b q^{2} / c d e, c / b, q / d, a q / d, b c / a, a^{2} q^{2} / b c d e, q / e, a q / e ; q\right)_{\infty}} \\
& \quad \times \sum_{k=0}^{\infty} \frac{\left(1-a b q^{1+2 k} / c d e\right)}{(1-a b q / c d e)} \frac{(a b q / c d e, a q / d e, a q / c e, a q / c d, b, b / a ; q)_{k}}{\left(q, b q / c, b q / d, b q / e, a q^{2} / c d e, a^{2} q^{2} / c d e ; q\right)_{k}} q^{k} \\
& \quad \times \sum_{n=-\infty}^{\infty} \frac{\left(1-a q^{2 n}\right)}{(1-a)} \frac{\left(f, g, b q^{k}, c d e q^{-1-k} / a ; q\right)_{n}}{\left(a g / f, a q / g, a q^{1-k} / b, a^{2} q^{2+k} / c d e ; q\right)_{n}}\left(\frac{a^{3} q^{2}}{b c d e f g}\right)^{n} \\
& +\operatorname{idem}(b ; c) .
\end{aligned}
$$

Now the inner sums can be evaluated by the ${ }_{6} \psi_{6}$ summation (3.1) and we obtain

$$
\begin{aligned}
& \frac{\left(b q / d, a q^{2} / c d e, a^{2} q^{2} / c d e, b q / e, a q / b e, c, c / a, a q / b d ; q\right)_{\infty}}{\left(a b q^{2} / c d e, c / b, q / d, a q / d, b c / a, a^{2} q^{2} / b c d e, q / e, a q / e ; q\right)_{\infty}} \\
& \quad \times \sum_{k=0}^{\infty} \frac{\left(1-a b q^{1+2 k} / c d e\right)}{(1-a b q / c d e)} \frac{(a b q / c d e, a q / d e, a q / c e, a q / c d, b, b / a ; q)_{k}}{\left(q, b q / c, b q / d, b q / e, a q^{2} / c d e, a^{2} q^{2} / c d e ; q\right)_{k}} q^{k}
\end{aligned}
$$




$$
\begin{array}{r}
\times \frac{\left(a q, a q / f g, a q^{1-k} / b f, a^{2} q^{2+k} / c \operatorname{def}, a q^{1-k} / b g, a^{2} q^{2+k} / c \operatorname{deg}, a^{2} q^{2} / b c d e, q, q / a ; q\right)_{\infty}}{\left(a q / f, a q / g, a q^{1-k} / b, a^{2} q^{2+k} / c d e, q / f, q / g, q^{1-k} / b, a q^{2+k} / c d e, a^{3} q^{2} / b c d e f g ; q\right)_{\infty}} \\
+\operatorname{idem}(b ; c),
\end{array}
$$

which can be simplified to

$$
\begin{gathered}
\frac{(b q / d, b q / e, a q / b e, c, c / a, a q / b d ; q)_{\infty}}{\left(a b q^{2} / c d e, c / b, q / d, a q / d, b c / a, q / e, a q / e ; q\right)_{\infty}} \\
\quad \times \frac{\left(a q, a q / f g, a q / b f, a^{2} q^{2} / c d e f, a q / b g, a^{2} q^{2} / c \operatorname{deg}, q, q / a ; q\right)_{\infty}}{\left(a q / f, a q / g, a q / b, q / f, q / g, q / b, a^{3} q^{2} / b c d e f g ; q\right)_{\infty}} \\
\times \sum_{k=0}^{\infty} \frac{\left(1-a b q^{1+2 k} / c d e\right)}{(1-a b q / c d e)} \frac{(a b q / c d e, a q / d e, a q / c e, a q / c d, b f / a, b g / a ; q)_{k}}{\left(q, b q / c, b q / d, b q / e, a^{2} q^{2} / c d e f, a^{2} q^{2} / c d e g ; q\right)_{k}}\left(\frac{a q}{e f}\right)^{k} \\
\quad+\operatorname{idem}(b ; c) .
\end{gathered}
$$

Now, to the ${ }_{8} \phi_{7}$ 's in (4.5) we apply Bailey's [7], Eq. (4.3)] transformation formula for a nonterminating ${ }_{8} \phi_{7}$ series (see [15, Eq. (2.10.1)]):

$$
\begin{aligned}
& { }_{8} \phi_{7}\left[\begin{array}{c}
a, q \sqrt{a},-q \sqrt{a}, b, c, d, e, f \\
\sqrt{a},-\sqrt{a}, a q / b, a q / c, a q / d, a q / e, a q / f
\end{array} ; q, \frac{a^{2} q^{2}}{b c d e f}\right] \\
& =\frac{(a q, a q / e f, \lambda q / e, \lambda q / f ; q)_{\infty}}{(a q / e, a q / f, \lambda q, \lambda q / e f ; q)_{\infty}} \\
& \times{ }_{8} \phi_{7}\left[\begin{array}{c}
\lambda, q \sqrt{\lambda},-q \sqrt{\lambda}, \lambda b / a, \lambda c / a, \lambda d / a, e, f \\
\sqrt{\lambda},-\sqrt{\lambda}, a q / b, a q / c, a q / d, \lambda q / e, \lambda q / f
\end{array} ; q, \frac{a q}{e f}\right],
\end{aligned}
$$

where $\lambda=a^{2} q / b c d$ and $\max (|a q / e f|,|\lambda q / e f|)<1$. The purpose of our application of this transformation is to obtain more symmetry. Hence, by (4.6) the expression in (4.5) is transformed into

$$
\begin{array}{r}
(b q / d, b q / e, a q / b e, c, c / a, a q / b d, a q, a q / b f, a q / b g, q, q / a, b q / f, b q / g ; q)_{\infty} \\
\hline\left(c / b, q / d, a q / d, b c / a, q / e, a q / e, a q / f, a q / g, a q / b, q / f, q / g, q / b, b^{2} q / a ; q\right)_{\infty} \\
\times{ }_{8} \phi_{7}\left[\begin{array}{rr}
b^{2} / a, q b / \sqrt{a},-q b / \sqrt{a}, b c / a, b d / a, b e / a, b f / a, b g / a & ; q, \frac{a^{3} q^{2}}{b c d e f g}
\end{array}\right] \\
b / \sqrt{a},-b / \sqrt{a}, b q / c, b q / d, b q / e, b q / f, b q / g \\
+\operatorname{idem}(b ; c),
\end{array}
$$

which already completes our derivation of M. Jackson's very-well-poised ${ }_{8} \psi_{8}$ transformation formula.

\section{A VERY-WELL-POISED ${ }_{10} \psi_{10}$ TRANSFORMATION}

In this section we derive with our method the following very-well-poised ${ }_{10} \psi_{10}$ transformation (cf. [15, Eq. (5.6.3)]):

$$
\begin{aligned}
& { }_{10} \psi_{10}\left[\begin{array}{c}
q \sqrt{a},-q \sqrt{a}, b, c, d, e, f, g, h, y \\
\sqrt{a},-\sqrt{a}, \frac{a q}{b}, \frac{a q}{c}, \frac{a q}{d}, \frac{a q}{e}, \frac{a q}{f}, \frac{a q}{g}, \frac{a q}{h}, \frac{a q}{y} ; q, \frac{a^{4} q^{3}}{b c d e f g h y}
\end{array}\right] \\
& =\frac{\left(q, a q, \frac{q}{a}, c, \frac{c}{a}, d, \frac{d}{a}, \frac{b q}{e}, \frac{b q}{f}, \frac{b q}{g}, \frac{b q}{h}, \frac{b q}{y}, \frac{a q}{b e}, \frac{a q}{b f}, \frac{a q}{b g}, \frac{a q}{b h}, \frac{a q}{b y} ; q\right)_{\infty}}{\left(\frac{q}{b}, \frac{q}{e}, \frac{q}{f}, \frac{q}{g}, \frac{q}{h}, \frac{q}{y}, \frac{a q}{b}, \frac{a q}{e}, \frac{a q}{f}, \frac{a q}{g}, \frac{a q}{h}, \frac{a q}{y}, \frac{b c}{a}, \frac{b d}{a}, \frac{c}{b}, \frac{d}{b}, \frac{b^{2} q}{a} ; q\right)_{\infty}} \\
& \times{ }_{10} \phi_{9}\left[\begin{array}{c}
\frac{b^{2}}{a}, \frac{a b}{\sqrt{a}},-\frac{q b}{\sqrt{a}}, \frac{b c}{a}, \frac{b d}{a}, \frac{b e}{a}, \frac{b f}{a}, \frac{b g}{a}, \frac{b h}{a}, \frac{b y}{a} \\
\frac{b}{\sqrt{a}},-\frac{b}{\sqrt{a}}, \frac{b q}{c}, \frac{b q}{d}, \frac{b q}{e}, \frac{b q}{f}, \frac{b q}{g}, \frac{b q}{h}, \frac{a^{4} q^{3}}{y}
\end{array} ; q, \frac{b c d e f g h y}{b c t}\right]
\end{aligned}
$$




$$
+\operatorname{idem}(b ; c, d)
$$

where the series either terminate, or $\left|a^{4} q^{3} / b c d e f g h y\right|<1$, for convergence. (The symbol "idem $(b ; c, d)$ " is explained in the introduction.)

Gasper and Rahman [15, Sec. 5.6] derive this ${ }_{10} \psi_{10}$ transformation formula by specializing a general transformation of Slater [33].

To derive this transformation formula with our method, we make use of a special case of Bailey's nonterminating ${ }_{8} \phi_{7}$ summation (4.2), namely the key identity (4.3), and of M. Jackson's ${ }_{8} \psi_{8}$ summation (4.1) which we just derived in the previous section. In the course of our derivation, we also make use of Jackson's terminating ${ }_{8} \phi_{7}$ summation (3.6), and of Bailey's nonterminating ${ }_{8} \phi_{7}$ summation (4.2) in its general form.

In identity (4.3), we first replace $d$ by $f$. Then we multiply both sides by

$$
\frac{\left(1-a q^{2 n}\right)}{(1-a)} \frac{(d, g, h, y ; q)_{n}}{(a q / d, a q / g, a q / h, a q / y ; q)_{n}}\left(\frac{a^{4} q^{3}}{b c d e f g h y}\right)^{n}
$$

and sum over all integers $n$.

On the right side we obtain

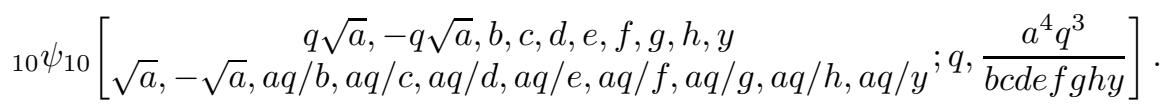

On the left side we obtain

$$
\begin{aligned}
& \frac{\left(b q / f, a q^{2} / c e f, a^{2} q^{2} / c e f, b q / e, a q / b e, c, c / a, a q / b f ; q\right)_{\infty}}{\left(a b q^{2} / c e f, c / b, q / f, a q / f, b c / a, a^{2} q^{2} / b c e f, q / e, a q / e ; q\right)_{\infty}} \\
& \times \sum_{n=-\infty}^{\infty} \frac{\left(1-a q^{2 n}\right)}{(1-a)} \frac{(d, g, h, y ; q)_{n}}{(a q / d, a q / g, a g / h, a q / y ; q)_{n}}\left(\frac{a^{4} q^{3}}{b c d e f g h y}\right)^{n} \\
& \quad \times \sum_{k=0}^{\infty} \frac{\left(1-a b q^{1+2 k} / c e f\right)}{(1-a b q / c e f)} \frac{(a b q / c e f, a q / e f, a q / c e, a q / c f ; q)_{k}}{(q, b q / c, b q / f, b q / e ; q)_{k}} \\
& \quad \times \frac{(b ; q)_{n+k}(c e f / a q ; q)_{n-k}}{\left(a^{2} q^{2} / c e f ; q\right)_{n+k}(a q / b ; q)_{n-k}}\left(\frac{b c e f}{a^{2} q}\right)^{k}+\operatorname{idem}(b ; c) .
\end{aligned}
$$

Next, we interchange summations in (5.2) and obtain

$$
\begin{gathered}
\frac{\left(b q / f, a q^{2} / c e f, a^{2} q^{2} / c e f, b q / e, a q / b e, c, c / a, a q / b f ; q\right)_{\infty}}{\left(a b q^{2} / c e f, c / b, q / f, a q / f, b c / a, a^{2} q^{2} / b c e f, q / e, a q / e ; q\right)_{\infty}} \\
\times \sum_{k=0}^{\infty} \frac{\left(1-a b q^{1+2 k} / c e f\right)}{(1-a b q / c e f)} \frac{(a b q / c e f, a q / e f, a q / c e, a q / c f, b, b / a ; q)_{k}}{\left(q, b q / c, b q / f, b q / e, a q^{2} / c e f, a^{2} q^{2} / c e f ; q\right)_{k}} q^{k} \\
\times \sum_{n=-\infty}^{\infty} \frac{\left(1-a q^{2 n}\right)}{(1-a)} \frac{\left(b q^{k}, d, g, h, y, c e f q^{-1-k} / a ; q\right)_{n}}{\left(a q^{1-k} / b, a q / d, a g / q, a q / h, a q / y, a^{2} q^{2+k} / c e f ; q\right)_{n}}\left(\frac{a^{4} q^{3}}{b c d e f g h y}\right)^{n} \\
\quad+\operatorname{idem}(b ; c) .
\end{gathered}
$$

Now to the inner sums we apply M. Jackson's ${ }_{8} \psi_{8}$ transformation (4.1) and we obtain

$$
\frac{\left(b q / f, a q^{2} / c e f, a^{2} q^{2} / c e f, b q / e, a q / b e, c, c / a, a q / b f ; q\right)_{\infty}}{\left(a b q^{2} / c e f, c / b, q / f, a q / f, b c / a, a^{2} q^{2} / b c e f, q / e, a q / e ; q\right)_{\infty}}
$$




$$
\begin{gathered}
\times \sum_{k=0}^{\infty} \frac{\left(1-a b q^{1+2 k} / c e f\right)}{(1-a b q / c e f)} \frac{(a b q / c e f, a q / e f, a q / c e, a q / c f, b, b / a ; q)_{k}}{\left(q, b q / c, b q / f, b q / e, a q^{2} / c e f, a^{2} q^{2} / c e f ; q\right)_{k}} q^{k} \\
\times\left[\frac{\left(q, a q, q / a, d, d / a, b q^{1+k} / g, b q^{1+k} / h, b q^{1+k} / y, a b q^{2+2 k} / c e f, a q^{1-k} / b g ; q\right)_{\infty}}{\left(q^{1-k} / b, q / g, q / h, q / y, a q^{2+k} / c e f, a q^{1-k} / b, a q / g, a q / h, a q / y, a^{2} q^{2+k} / c e f ; q\right)_{\infty}}\right. \\
\times \frac{\left(a q^{1-k} / b h, a q^{1-k} / b y, a^{2} q / b c e f ; q\right)_{\infty}}{\left(d q^{-k} / b, b d q^{k} / a, b^{2} q^{1+2 k} / a ; q\right)_{\infty}} \sum_{n=0}^{\infty} \frac{\left(1-b^{2} q^{2 k+2 n} / a\right)}{\left(1-b^{2} q^{2 k} / a\right)} \frac{\left(b^{2} q^{2 k} / a, b d q^{k} / a ; q\right)_{n}}{\left(q, b q^{1+k} / d ; q\right)_{n}} \\
\quad \times \frac{\left(b g q^{k} / a, b h q^{k} / a, b y q^{k} / a, b c e f / a^{2} q ; q\right)_{n}}{\left(b q^{1+k} / g, b q^{1+k} / h, b q^{1+k} / y, a b q^{2+2 k} / c e f ; q\right)_{n}}\left(\frac{a^{4} q^{3}}{b c d e f g h y}\right)^{n} \\
+\frac{\left(q, a q, q / a, b q^{k}, b q^{k} / a, d q / g, d q / h, d q / y, a d q^{2+2 k} / c e f, a q / d g ; q\right)_{\infty}}{\left(q / d, q / g, q / h, q / y, a q^{2+k} / c e f, a q / d, a q / g, a q / h, a q / y, a^{2} q^{2+k} / c e f ; q\right)_{\infty}} \\
\times \frac{\left(a q / d h, a q / d y, a^{2} q^{2+k} / c d e f ; q\right)_{\infty}}{\left(b q^{k} / d, b d q^{k} / a, d^{2} q / a ; q\right)_{\infty}} \sum_{n=0}^{\infty} \frac{\left(1-d^{2} q^{2 n} / a\right)}{\left(1-d^{2} / a\right)} \frac{\left(d^{2} / a, d b q^{k} / a ; q\right)_{n}}{\left(q, d q^{1-k} / b ; q\right)_{n}} \\
\left.\times \frac{\left(d g / a, d h / a, d y / a, c d e f q^{-1-k} / a^{2} ; q\right)_{n}}{\left(d q / g, d q / h, d q / y, a d q^{2+k} / c e f ; q\right)_{n}}\left(\frac{a^{4} q^{3}}{b c d e f g h y}\right)^{n}\right]+\operatorname{idem}(b ; c),
\end{gathered}
$$

which can be (slightly) simplified to

$$
\begin{gathered}
{\left[\frac{(b q / f, b q / e, a q / b e, c, c / a, a q / b f, q, a q, q / a, d, d / a, b q / g, b q / h, b q / y ; q)_{\infty}}{(c / b, q / f, a q / f, b c / a, q / e, a q / e, q / b, q / g, q / h, q / y, a q / b, a q / g, a q / h, a q / y ; q)_{\infty}}\right.} \\
\times \frac{(a q / b g, a q / b h, a q / b y ; q)_{\infty}}{\left(d / b, b d / a, b^{2} q / a ; q\right)_{\infty}} \sum_{k=0}^{\infty} \frac{\left(1-a b q^{1+2 k} / c e f\right)}{(1-a b q / c e f)} \frac{(a b q / c e f, a q / e f, a q / c e ; q)_{k}}{(q, b q / c, b q / f ; q)_{k}} \\
\times \frac{(a q / c f, b d / a, b g / a, b h / a, b y / a ; q)_{k}\left(b^{2} q / a ; q\right)_{2 k}}{(b q / e, b q / d, b q / g, b q / h, b q / y ; q)_{k}\left(a b^{2} q^{2} / c e f ; q\right)_{2 k}}\left(\frac{a^{2} q^{2}}{d g h y}\right)^{k} \\
\times \sum_{n=0}^{\infty} \frac{\left(1-b^{2} q^{2 k+2 n} / a\right)}{\left(1-b^{2} q^{2 k} / a\right)} \frac{\left(b^{2} q^{2 k} / a, b d q^{k} / a, b g q^{k} / a, b h q^{k} / a ; q\right)_{n}}{\left(q, b q^{1+k} / d, b q^{1+k} / g, b q^{1+k} / h ; q\right)_{n}} \\
\times \frac{\left(b y q^{k} / a, b c e f / a^{2} q ; q\right)_{n}}{\left(b q^{1+k} / y, a b q^{2+2 k} / c e f ; q\right)_{n}}\left(\frac{a^{4} q^{3}}{b c d e f g h y}\right)^{n} \\
+\frac{(b q / f, b q / e, a q / b e, c, c / a, a q / b f, q, a q, q / a, b, b / a, d q / g ; q)_{\infty}}{\left(a b q^{2} / c e f, c / b, q / f, a q / f, b c / a, a^{2} q^{2} / b c e f, q / e, a q / e, q / d, q / g, q / h, q / y ; q\right)_{\infty}} \\
\times \frac{\left(d q / h, d q / y, a d q^{2} / c e f, a q / d g, a q / d h, a q / d y, a^{2} q^{2} / c d e f ; q\right)_{\infty}}{\left(a q / d, a q / g, a q / h, a q / y, b / d, b d / a, d d^{2} q / a ; q\right)_{\infty}} \\
\times \sum_{k=0}^{\infty} \frac{\left(1-a b q^{1+2 k} / c e f\right)}{(1-a b q / c e f)} \frac{(a b q / c e f, a q / e f, a q / c e, a q / c f, b / d, b d / a ; q)_{k}}{\left(q, b q / c, b q / f, b q / e, a d q^{2} / c e f, a^{2} q^{2} / c d e f ; q\right)_{k}} q^{k} \\
\times \sum_{n=0}^{\infty} \frac{\left(1-d^{2} q^{2 n} / a\right)}{\left(1-d^{2} / a\right)} \frac{\left(d^{2} / a, d b q^{k} / a, d g / a, d h / a ; q\right)_{n}}{\left(q, d q^{1-k} / b, d g / a, d h / a ; q\right)_{n}} \\
\left.\times \frac{\left(d y / a, c d e f q^{-1-k} / a^{2} ; q\right)_{n}}{\left(d q / y, a d q^{2+k} / c e f ; q\right)_{n}}\left(\frac{a^{4} q^{3}}{b c d e f g h y}\right)^{n}\right]+\mathrm{idem}(b ; c) . \quad(5.3)
\end{gathered}
$$


We have in (5.3) a sum of four double sums. Accordingly, for more clarity, let us write the whole expression in (5.3) as

$$
T_{1}(b, c)+T_{2}(b, c)+U_{1}(b, c)+U_{2}(b, c),
$$

where, by definition of "idem", $U_{1}(b, c)=T_{1}(c, b)$ and $U_{2}(b, c)=T_{2}(c, b)$. Below, we will selectively perform manipulations with the terms $T_{1}, T_{2}, U_{1}$, and $U_{2}$.

To evaluate $T_{1}(b, c)$ (and hence also $U_{1}(b, c)$ ), we first shift the index $n$ of the inner sum in $T_{1}(b, c)$ (or, equivalently, in the first term in (5.3)) by $-k$ and then interchange the double sum. Symbolically, we apply

$$
\sum_{k=0}^{\infty} \sum_{n=0}^{\infty} f(n, k)=\sum_{n=0}^{\infty} \sum_{k=0}^{n} f(n-k, k) .
$$

Thus, we obtain (using some elementary identities for $q$-shifted factorials)

$$
\begin{gathered}
T_{1}(b, c)=\frac{(b q / f, b q / e, a q / b e, c, c / a, a q / b f, q, a q, q / a, d, d / a ; q)_{\infty}}{(c / b, q / f, a q / f, b c / a, q / e, a q / e, q / b, q / g, q / h, q / y, a q / b ; q)_{\infty}} \\
\times \frac{(b q / g, b q / h, b q / y, a q / b g, a q / b h, a q / b y ; q)_{\infty}}{\left(a q / g, a q / h, a q / y, d / b, b d / a, b^{2} q / a ; q\right)_{\infty}} \\
\times \sum_{n=0}^{\infty} \frac{\left(1-b^{2} q^{2 n} / a\right)}{\left(1-b^{2} / a\right)} \frac{\left(b^{2} / a, b d / a, b g / a, b h / a, b y / a, b c e f / a^{2} q ; q\right)_{n}}{\left(q, b q / d, b q / g, b q / h, b q / y, a b q^{2} / c e f ; q\right)_{n}}\left(\frac{a^{4} q^{3}}{b c d e f g h y}\right)^{n} \\
\times \sum_{k=0}^{n} \frac{\left(1-a b q^{1+2 k} / c e f\right)}{(1-a b q / c e f)} \frac{\left(a b q / c e f, a q / e f, a q / c e, a q / c f, b^{2} q^{n} / a, q^{-n} ; q\right)_{k}}{\left(q, b q / c, b q / f, b q / e, a^{2} q^{2-n} / b c e f, a b q^{2+n} / c e f ; q\right)_{k}} q^{k} .
\end{gathered}
$$

Now the inner sum can be evaluated by Jackson's terminating ${ }_{8} \phi_{7}$ summation (3.6), which simplifies the last expression, $T_{1}(b, c)$, to

$(b q / f, b q / e, a q / b e, c, c / a, a q / b f, q, a q, q / a, d, d / a, b q / g, b q / h, b q / y ; q)_{\infty}$ $\overline{(c / b, q / f, a q / f, b c / a, q / e, a q / e, q / b, q / g, q / h, q / y, a q / b, a q / g, a q / h, a q / y ; q)_{\infty}}$

$$
\begin{gathered}
\times \frac{(a q / b g, a q / b h, a q / b y ; q)_{\infty}}{\left(d / b, b d / a, b^{2} q / a ; q\right)_{\infty}} \sum_{n=0}^{\infty} \frac{\left(1-b^{2} q^{2 n} / a\right)}{\left(1-b^{2} / a\right)} \frac{\left(b^{2} / a, b c / a, b d / a, b e / a ; q\right)_{n}}{(q, b q / c, b q / d, b q / e ; q)_{n}} \\
\times \frac{(b f / a, b g / a, b h / a, b y / a, ; q)_{n}}{(b q / f, b q / g, b q / h, b q / y ; q)_{n}}\left(\frac{a^{4} q^{3}}{b c d e f g h y}\right)^{n} .
\end{gathered}
$$

Next, we consider $T_{2}(b, c)$ and $U_{2}(b, c)$. By interchanging the double sums in $T_{2}(b, c)$ and in $U_{2}(b, c)$ we obtain

$$
\begin{gathered}
T_{2}(b, c)+U_{2}(b, c)=\frac{(b q / f, b q / e, a q / b e, c, c / a, a q / b f, q, a q, q / a ; q)_{\infty}}{\left(a b q^{2} / c e f, c / b, q / f, a q / f, b c / a, a^{2} q^{2} / b c e f, q / e, a q / e, q / d ; q\right)_{\infty}} \\
\times \frac{\left(b, b / a, d q / g, d q / h, d q / y, a d q^{2} / c e f, a q / d g, a q / d h, a q / d y, a^{2} q^{2} / c d e f ; q\right)_{\infty}}{\left(q / g, q / h, q / y, a q / d, a q / g, a q / h, a q / y, b / d, b d / a, d^{2} q / a ; q\right)_{\infty}} \\
\times \sum_{n=0}^{\infty} \frac{\left(1-d^{2} q^{2 n} / a\right)}{\left(1-d^{2} / a\right)} \frac{\left(d^{2} / a, d b / a, d g / a, d h / a, d y / a, c d e f / a^{2} q ; q\right)_{n}}{\left(q, d q / b, d g / a, d h / a, d q / y, a d q^{2} / c e f ; q\right)_{n}}\left(\frac{a^{4} q^{3}}{b c d e f g h y}\right)^{n} \\
\times \sum_{k=0}^{\infty} \frac{\left(1-a b q^{1+2 k} / c e f\right)}{(1-a b q / c e f)} \frac{\left(a b q / c e f, a q / e f, a q / c e, a q / c f, b q^{-n} / d, b d q^{n} / a ; q\right)_{k}}{\left(q, b q / c, b q / f, b q / e, a d q^{2+n} / c e f, a^{2} q^{2-n} / c d e f ; q\right)_{k}} q^{k} \\
+\operatorname{idem}(b ; c) .
\end{gathered}
$$


Now, to the inner sum of the first double sum (but not of the second!) in (5.7) we apply Bailey's nonterminating ${ }_{8} \phi_{7}$ summation (4.2), i.e., specifically we apply

$$
\begin{gathered}
\sum_{k=0}^{\infty} \frac{\left(1-a b q^{1+2 k} / c e f\right)}{(1-a b q / c e f)} \frac{\left(a b q / c e f, a q / e f, a q / c e, a q / c f, b q^{-n} / d, b d q^{n} / a ; q\right)_{k}}{\left(q, b q / c, b q / f, b q / e, a d q^{2+n} / c e f, a^{2} q^{2-n} / c d e f ; q\right)_{k}} q^{k} \\
=\frac{\left(a b q^{2} / c e f, c / b, b c / a, d q^{1+n} / f, a q^{1-n} / d f, d q^{1+n} / e, a q^{1-n} / d e, a^{2} q^{2} / b c e f ; q\right)_{\infty}}{\left(b q / f, b q / e, a d q^{2+n} / c e f, a^{2} q^{2-n} / c d e f, a q / b e, a q / b f, c q^{-n} / d, c d q^{n} / a ; q\right)_{\infty}} \\
-\frac{\left(a b q^{2} / c e f, a q / c e, a q / c f, b q^{-n} / d, b d q^{n} / a, c / b, c q / f, c q / e, a d q^{2+n} / b e f ; q\right)_{\infty}}{\left(b / c, b q / f, b q / e, a d q^{2+n} / c e f, a^{2} q^{2-n} / c d e f, a q / b e, a q / b f, c q^{-n} / d, c d q^{n} / a ; q\right)_{\infty}} \\
\times \frac{\left(a^{2} q^{2-n} / b d e f ; q\right)_{\infty}}{\left(a c q^{2} / b e f ; q\right)_{\infty}} \sum_{k=0}^{\infty} \frac{\left(1-a c q^{1+2 k} / b e f\right)}{(1-a c q / b e f)} \frac{(a c q / b e f, a q / e f, a q / b e ; q)_{k}}{(q, c q / b, c q / f ; q)_{k}} \\
\times \frac{\left(a q / b f, c q^{-n} / d, c d q^{n} / a ; q\right)_{k}}{\left(c q / e, a d q^{2+n} / b e f, a^{2} q^{2-n} / b d e f ; q\right)_{k}} q^{k} .
\end{gathered}
$$

The result of the application of this (two term) summation is that the first term in (5.7), $T_{2}(b, c)$, is split into two parts, one single sum $T_{2}^{\prime}(b, c)$ and one double sum $T_{2}^{\prime \prime}(b, c)$. Formally, we have

$$
T_{2}(b, c)+U_{2}(b, c)=\left[T_{2}^{\prime}(b, c)+T_{2}^{\prime \prime}(b, c)\right]+U_{2}(b, c) .
$$

But $T_{2}^{\prime \prime}(b, c)$ is precisely $-U_{2}(b, c)$ (as can be readily checked), so two terms cancel, thus

$$
T_{2}(b, c)+U_{2}(b, c)=T_{2}^{\prime}(b, c) .
$$

Now, the last expression, $T_{2}^{\prime}(b, c)$, can be simplified to

$$
\begin{gathered}
\frac{(c, c / a, q, a q, q / a, b, b / a, d q / g, d q / h, d q / y, a q / d g, a q / d h, a q / d y, d q / f ; q)_{\infty}}{(q / f, a q / f, q / e, a q / e, q / d, q / g, q / h, q / y, a q / d, a q / g, a q / h, a q / y, b / d, b d / a ; q)_{\infty}} \\
\times \frac{(a q / d f, d q / e, a q / d e ; q)_{\infty}}{\left(d^{2} q / a, c / d, c d / a ; q\right)_{\infty}} \sum_{n=0}^{\infty} \frac{\left(1-d^{2} q^{2 n} / a\right)}{\left(1-d^{2} / a\right)} \frac{\left(d^{2} / a, d b / a, d c / a, d e / a ; q\right)_{n}}{(q, d q / b, d q / c, d q / e ; q)_{n}} \\
\times \frac{(d f / a, d g / a, d h / a, d y / a ; q)_{n}}{(d q / f, d g / a, d h / a, d q / y ; q)_{n}}\left(\frac{a^{4} q^{3}}{b c d e f g h y}\right)^{n} \cdot
\end{gathered}
$$

It is easy to see that (5.9) equals (5.6) where $b$ and $d$ are interchanged. Collecting all terms, according to (5.4), completes our derivation of the very-well-poised ${ }_{10} \psi_{10}$ transformation in (5.1).

\section{Slater's transformations For Bilateral Well-Poised SERIES}

In view of the success of our (more or less) elaborate but elementary derivation of the very-well-poised ${ }_{10} \psi_{10}$ transformation in the preceding section, we are encouraged to go for more. In fact, the same machinery applies, together with induction, to prove the following general very-well-poised ${ }_{2 r} \psi_{2 r}$ transformation formula due to Slater 33]. For $r \geq 3$,

$$
\begin{aligned}
{ }_{2 r} \psi_{2 r} & {\left[\begin{array}{l}
q \sqrt{a},-q \sqrt{a}, b_{3}, b_{4}, \ldots, b_{2 r} \\
\sqrt{a},-\sqrt{a}, \frac{a q}{b_{3}}, \frac{a q}{b_{4}}, \ldots, \frac{a q}{b_{2 r}} ; q, \frac{a^{r-1} q^{r-2}}{b_{3} \ldots b_{2 r}}
\end{array}\right] } \\
= & \frac{\left(q, a q, \frac{q}{a}, b_{4}, \ldots, b_{r}, \frac{b_{4}}{a}, \ldots, \frac{b_{r}}{a}, \frac{b_{3} q}{b_{r+1}}, \ldots, \frac{b_{3} q}{b_{2 r}}, \frac{a q}{b_{3} b_{r+1}}, \ldots, \frac{a q}{b_{3} b_{2 r}} ; q\right)_{\infty}}{\left(\frac{q}{b_{3}}, \frac{q}{b_{r+1}}, \ldots, \frac{q}{b_{2 r}}, \frac{a q}{b_{3}}, \frac{a q}{b_{r+1}}, \ldots, \frac{a q}{b_{2 r}}, \frac{b_{4}}{b_{3}}, \ldots, \frac{b_{r}}{b_{3}}, \frac{b_{3} b_{4}}{a}, \ldots, \frac{b_{3} b_{r}}{a}, \frac{b_{3}^{2} q}{a} ; q\right)_{\infty}}
\end{aligned}
$$




$$
\begin{aligned}
& \times{ }_{2 r} \phi_{2 r-1}\left[\begin{array}{c}
\frac{b_{3}^{2}}{a}, \frac{q b_{3}}{\sqrt{a}},-\frac{q b_{3}}{\sqrt{a}}, \frac{b_{3} b_{4}}{a}, \frac{b_{3} b_{5}}{a}, \ldots, \frac{b_{3} b_{2 r}}{a} \\
\frac{b_{3}}{\sqrt{a}},-\frac{b_{3}}{\sqrt{a}}, \frac{b_{3} q}{b_{4}}, \frac{b_{3} q}{b_{5}}, \ldots, \frac{b_{3} q}{b_{2 r}}
\end{array} ; q \frac{a^{r-1} q^{r-2}}{b_{3} \ldots b_{2 r}}\right] \\
& +\operatorname{idem}\left(b_{3} ; b_{4}, \ldots, b_{r}\right),
\end{aligned}
$$

where the series either terminate, or $\left|a^{r-1} q^{r-2} / b_{3} \ldots b_{2 r}\right|<1$, for convergence. (The symbol "idem $\left(b_{3} ; b_{4}, \ldots, b_{r}\right)$ " is explained in the introduction.)

Slater [33] first obtained this transformation formula (or rather the more general one in (6.11) ) by extending Sears' 32 general transformation for (unilateral) basic hypergeometric series. Not much later, she 34 gave a direct proof using a basic Barnes-type contour integral.

Here, we provide an inductive proof of (6.1) following closely the analysis of our derivation of the very-well-poised ${ }_{10} \psi_{10}$ transformation in Section 5 .

The $r=3$ case of (6.1) is readily verified using the ${ }_{6} \psi_{6}$ and ${ }_{6} \phi_{5}$ summations in (3.1) and in (3.2), respectively. We have also shown the $r=4$ and $r=5$ cases in Sections 4 and 5. So we can assume that $r>5$.

Now suppose that the formula in (6.1) is already shown for any integer $t$ where $3 \leq t<r$. To show the transformation for $t=r$, we first perform in the key identity (4.3) the substitutions $b \mapsto b_{3}, c \mapsto b_{4}, d \mapsto b_{r+2}$, and $e \mapsto b_{r+1}$. In the resulting equation, we multiply both sides by

$$
\frac{\left(1-a q^{2 n}\right)}{(1-a)} \frac{\left(b_{5}, \ldots, b_{r}, b_{r+3}, \ldots, b_{2 r} ; q\right)_{n}}{\left(a q / b_{5}, \ldots, a q / b_{r}, a q / b_{r+3}, \ldots, a q / b_{2 r} ; q\right)_{n}}\left(\frac{a^{r-1} q^{r-2}}{b_{3} \ldots b_{2 r}}\right)^{n}
$$

and sum over all integers $n$.

On the right side we obtain

$$
{ }_{2 r} \psi_{2 r}\left[\begin{array}{c}
q \sqrt{a},-q \sqrt{a}, b_{3}, b_{4}, \ldots, b_{2 r} \\
\sqrt{a},-\sqrt{a}, a q / b_{3}, a q / b_{4}, \ldots, a q / b_{2 r}
\end{array} ; q, \frac{a^{r-1} q^{r-2}}{b_{3} \ldots b_{2 r}}\right] .
$$

On the left side we obtain

$$
\begin{gathered}
\frac{\left(b_{3} q / b_{r+2}, a q^{2} / b_{4} b_{r+1} b_{r+2}, a^{2} q^{2} / b_{4} b_{r+1} b_{r+2}, b_{3} q / b_{r+1} ; q\right)_{\infty}}{\left(a b_{3} q^{2} / b_{4} b_{r+1} b_{r+2}, b_{4} / b_{3}, q / b_{r+2}, a q / b_{r+2} ; q\right)_{\infty}} \\
\times \frac{\left(a q / b_{3} b_{r+1}, b_{4}, b_{4} / a, a q / b_{3} b_{r+2} ; q\right)_{\infty}}{\left(b_{3} b_{4} / a, a^{2} q^{2} / b_{3} b_{4} b_{r+1} b_{r+2}, q / b_{r+1}, a q / b_{r+1} ; q\right)_{\infty}} \\
\times \sum_{n=-\infty}^{\infty} \frac{\left(1-a q^{2 n}\right)}{(1-a)} \frac{\left(b_{5}, \ldots, b_{r}, b_{r+3}, \ldots, b_{2 r} ; q\right)_{n}}{\left(a q / b_{5}, \ldots, a q / b_{r}, a q / b_{r+3}, \ldots, a q / b_{2 r} ; q\right)_{n}}\left(\frac{a^{r-1} q^{r-2}}{b_{3} \ldots b_{2 r}}\right)^{n} \\
\times \sum_{k=0}^{\infty} \frac{\left(1-a b_{3} q^{1+2 k} / b_{4} b_{r+1} b_{r+2}\right)}{\left(1-a b_{3} q / b_{4} b_{r+1} b_{r+2}\right)} \frac{\left(a b_{3} q / b_{4} b_{r+1} b_{r+2}, a q / b_{r+1} b_{r+2}, a q / b_{4} b_{r+1} ; q\right)_{k}}{\left(q, b_{3} q / b_{4}, b_{3} q / b_{r+2} ; q\right)_{k}} \\
\times \frac{\left(a q / b_{4} b_{r+2} ; q\right)_{k}\left(b_{3} ; q\right)_{n+k}\left(b_{4} b_{r+1} b_{r+2} / a q ; q\right)_{n-k}}{\left(b_{3} q / b_{r+1} ; q\right)_{k}\left(a^{2} q^{2} / b_{4} b_{r+1} b_{r+2} ; q\right)_{n+k}\left(a q / b_{3} ; q\right)_{n-k}}\left(\frac{b_{3} b_{4} b_{r+1} b_{r+2}}{a^{2} q}\right)^{k} \\
+\operatorname{idem}\left(b_{3} ; b_{4}\right) .
\end{gathered}
$$

Next, we interchange summations in (6.2) and obtain

$$
\begin{array}{r}
\frac{\left(b_{3} q / b_{r+2}, a q^{2} / b_{4} b_{r+1} b_{r+2}, a^{2} q^{2} / b_{4} b_{r+1} b_{r+2}, b_{3} q / b_{r+1} ; q\right)_{\infty}}{\left(a b_{3} q^{2} / b_{4} b_{r+1} b_{r+2}, b_{4} / b_{3}, q / b_{r+2}, a q / b_{r+2} ; q\right)_{\infty}} \\
\times \frac{\left(a q / b_{3} b_{r+1}, b_{4}, b_{4} / a, a q / b_{3} b_{r+2} ; q\right)_{\infty}}{\left(b_{3} b_{4} / a, a^{2} q^{2} / b_{3} b_{4} b_{r+1} b_{r+2}, q / b_{r+1}, a q / b_{r+1} ; q\right)_{\infty}}
\end{array}
$$




$$
\begin{gathered}
\times \sum_{k=0}^{\infty} \frac{\left(1-a b_{3} q^{1+2 k} / b_{4} b_{r+1} b_{r+2}\right)}{\left(1-a b_{3} q / b_{4} b_{r+1} b_{r+2}\right)} \frac{\left(a b_{3} q / b_{4} b_{r+1} b_{r+2}, a q / b_{r+1} b_{r+2}, a q / b_{4} b_{r+1} ; q\right)_{k}}{\left(q, b_{3} q / b_{4}, b_{3} q / b_{r+2} ; q\right)_{k}} \\
\times \frac{\left(a q / b_{4} b_{r+2}, b_{3}, b_{3} / a ; q\right)_{k}}{\left(b_{3} q / b_{r+1}, a q^{2} / b_{4} b_{r+1} b_{r+2}, a^{2} q^{2} / b_{4} b_{r+1} b_{r+2} ; q\right)_{k}} q^{k} \\
\sum_{n=-\infty}^{\infty} \frac{\left(1-a q^{2 n}\right)}{(1-a)} \frac{\left(b_{3} q^{k}, b_{5}, \ldots, b_{r}, b_{r+3}, \ldots, b_{2 r} ; q\right)_{n}}{\left(a q^{1-k} / b_{3}, a q / b_{5}, \ldots, a q / b_{r}, a q / b_{r+3}, \ldots, a q / b_{2 r} ; q\right)_{n}} \\
\quad \times \frac{\left(b_{4} b_{r+1} b_{r+2} q^{-1-k} / a ; q\right)_{n}}{\left(a^{2} q^{2+k} / b_{4} b_{r+1} b_{r+2} ; q\right)_{n}}\left(\frac{a^{r-1} q^{r-2}}{b_{3} \ldots b_{2 r}}\right)^{n}+\operatorname{idem}\left(b_{3} ; b_{4}\right) .
\end{gathered}
$$

Now, to the inner sums we apply the inductive hypothesis (i.e., the $r \mapsto r-1$ case of (6.1)), and we obtain

$$
\begin{aligned}
& \frac{\left(b_{3} q / b_{r+2}, a q^{2} / b_{4} b_{r+1} b_{r+2}, a^{2} q^{2} / b_{4} b_{r+1} b_{r+2}, b_{3} q / b_{r+1} ; q\right)_{\infty}}{\left(\left(a b_{3} q^{2} / b_{4} b_{r+1} b_{r+2}, b_{4} / b_{3}, q / b_{r+2,} a q / b_{r+2} ;\right)_{\infty}\right.} \\
& \left(a b_{3} q^{2} / b_{4} b_{r+1} b_{r+2}, b_{4} / b_{3}, q / b_{r+2}, a q / b_{r+2} ; q\right)_{\infty} \\
& \times \frac{\left(a q / b_{3} b_{r+1}, b_{4}, b_{4} / a, a q / b_{3} b_{r+2} ; q\right)_{\infty}}{\left(b_{3} b_{4} / a, a^{2} q^{2} / b_{3} b_{4} b_{r+1} b_{r+2}, q / b_{r+1}, a q / b_{r+1} ; q\right)_{\infty}} \\
& \times \sum_{k=0}^{\infty} \frac{\left(1-a b_{3} q^{1+2 k} / b_{4} b_{r+1} b_{r+2}\right)}{\left(1-a b_{3} q / b_{4} b_{r+1} b_{r+2}\right)} \frac{\left(a b_{3} q / b_{4} b_{r+1} b_{r+2}, a q / b_{r+1} b_{r+2}, a q / b_{4} b_{r+1} ; q\right)_{k}}{\left(q, b_{3} q / b_{4}, b_{3} q / b_{r+2} ; q\right)_{k}} \\
& \times \frac{\left(a q / b_{4} b_{r+2}, b_{3}, b_{3} / a ; q\right)_{k}}{\left(b_{3} q / b_{r+1}, a q^{2} / b_{4} b_{r+1} b_{r+2}, a^{2} q^{2} / b_{4} b_{r+1} b_{r+2} ; q\right)_{k}} q^{k} \\
& \times\left[\frac{\left(q, a, q / a, b_{5}, \ldots, b_{r}, b_{5} / a, \ldots, b_{r} / a ; q\right)_{\infty}}{\left(q^{1-k} / b_{3}, q / b_{r+3}, \ldots, q / b_{2 r}, a q^{2+k} / b_{4} b_{r+1} b_{r+2} ; q\right)_{\infty}}\right. \\
& \times \frac{\left(b_{3} q^{1+k} / b_{r+3}, \ldots, b_{3} q^{1+k} / b_{2 r}, a b_{3} q^{2+2 k} / b_{4} b_{r+1} b_{r+2} ; q\right)_{\infty}}{\left(a q^{1-k} / b_{3}, a q / b_{r+3}, \ldots, a q / b_{2 r}, a^{2} q^{2+k} / b_{4} b_{r+1} b_{r+2} ; q\right)_{\infty}} \\
& \times \frac{\left(a q^{1-k} / b_{3} b_{r+3}, \ldots, a q^{1-k} / b_{3} b_{2 r}, a^{2} q^{2} / b_{3} b_{4} b_{r+1} b_{r+2} ; q\right)_{\infty}}{\left(b_{5} q^{-k} / b_{3}, \ldots, b_{r} q^{-k} / b_{3}, b_{3} b_{5} q^{k} / a, \ldots, b_{3} b_{r} q^{k} / a, b_{3}^{2} q^{1+2 k} / a ; q\right)_{\infty}} \\
& \times \sum_{n=0}^{\infty} \frac{\left(1-b_{3}^{2} q^{2 k+2 n} / a\right)}{\left(1-b_{3}^{2} q^{2 k} / a\right)} \frac{\left(b_{3}^{2} q^{2 k} / a, b_{3} b_{5} q^{k} / a, \ldots, b_{3} b_{r} q^{k} / a ; q\right)_{n}}{\left(q, b_{3} q^{1+k} / b_{5}, \ldots, b_{3} q^{1+k} / b_{r} ; q\right)_{n}} \\
& \times \frac{\left(b_{3} b_{r+3} q^{k} / a, \ldots, b_{3} b_{2 r} q^{k} / a, b_{3} b_{4} b_{r+1} b_{r+2} / a^{2} q ; q\right)_{n}}{\left(b_{3} q^{1+k} / b_{r+3}, \ldots, b_{3} q^{1+k} / b_{2 r}, a b_{3} q^{2+2 k} / b_{4} b_{r+1} b_{r+2} ; q\right)_{n}}\left(\frac{a^{r-1} q^{r-2}}{b_{3} \ldots b_{2 r}}\right)^{n} \\
& +\left(\frac{\left(q, a, q / a, b_{3} q^{k}, b_{6}, \ldots, b_{r}, b_{3} q^{k} / a, b_{6} / a, \ldots, b_{r} / a ; q\right)_{\infty}}{\left(q / b_{5}, q / b_{r+3}, \ldots, q / b_{2 r}, a q^{2+k} / b_{4} b_{r+1} b_{r+2} ; q\right)_{\infty}}\right. \\
& \times \frac{\left(b_{5} q / b_{r+3}, \ldots, b_{5} q / b_{2 r}, a b_{5} q^{2+k} / b_{4} b_{r+1} b_{r+2} ; q\right)_{\infty}}{\left(a q / b_{5}, a q / b_{r+3}, \ldots, a q / b_{2 r}, a^{2} q^{2+k} / b_{4} b_{r+1} b_{r+2} ; q\right)_{\infty}} \\
& \times \frac{\left(a q / b_{5} b_{r+3}, \ldots, a q / b_{5} b_{2 r}, a^{2} q^{2+k} / b_{4} b_{5} b_{r+1} b_{r+2} ; q\right)_{\infty}}{\left(b_{3} q^{k} / b_{5}, b_{6} / b_{5}, \ldots, b_{r} / b_{5}, b_{3} b_{5} q^{k} / a, b_{5} b_{6} / a, \ldots, b_{5} b_{r} / a, b_{5}^{2} q / a ; q\right)_{\infty}} \\
& \times \sum_{n=0}^{\infty} \frac{\left(1-b_{5}^{2} q^{2 n} / a\right)}{\left(1-b_{5}^{2} / a\right)} \frac{\left(b_{5}^{2} / a, b_{3} b_{5} q^{k} / a, b_{5} b_{6} / a, \ldots, b_{5} b_{r} / a ; q\right)_{n}}{\left(q, b_{5} q^{1-k} / b_{3}, b_{5} q / b_{6}, \ldots, b_{5} q / b_{r} ; q\right)_{n}} \\
& \times \frac{\left(b_{5} b_{r+3} / a, \ldots, b_{5} b_{2 r} / a, b_{4} b_{5} b_{r+1} b_{r+2} q^{-1-k} / a^{2} ; q\right)_{n}}{\left(b_{5} q / b_{r+3}, \ldots, b_{5} q / b_{2 r}, a b_{5} q^{2+k} / b_{4} b_{r+1} b_{r+2} ; q\right)_{n}}\left(\frac{a^{r-1} q^{r-2}}{b_{3} \ldots b_{2 r}}\right)^{n}
\end{aligned}
$$




$$
\left.\left.+\operatorname{idem}\left(b_{5} ; b_{6}, \ldots, b_{r}\right)\right)\right]+\operatorname{idem}\left(b_{3} ; b_{4}\right),
$$

which can be simplified to

$$
\begin{aligned}
& {\left[\frac{\left(b_{3} q / b_{r+2}, b_{3} q / b_{r+1}, a q / b_{3} b_{r+1}, b_{4}, b_{4} / a, a q / b_{3} b_{r+2}, q, a, q / a, b_{5}, \ldots, b_{r} ; q\right)_{\infty}}{\left(b_{4} / b_{3}, q / b_{r+2}, a q / b_{r+2}, b_{3} b_{4} / a, q / b_{r+1}, a q / b_{r+1}, q / b_{3}, q / b_{r+3}, \ldots, q / b_{2 r} ; q\right)_{\infty}}\right.} \\
& \times \frac{\left(b_{5} / a, \ldots, b_{r} / a, b_{3} q / b_{r+3}, \ldots, b_{3} q / b_{2 r}, a q / b_{3} b_{r+3}, \ldots, a q / b_{3} b_{2 r} ; q\right)_{\infty}}{\left(a q / b_{3}, a q / b_{r+3}, \ldots, a q / b_{2 r}, b_{5} / b_{3}, \ldots, b_{r} / b_{3}, b_{3} b_{5} / a, \ldots, b_{3} b_{r} / a, b_{3}^{2} q / a ; q\right)_{\infty}} \\
& \times \sum_{k=0}^{\infty} \frac{\left(1-a b_{3} q^{1+2 k} / b_{4} b_{r+1} b_{r+2}\right)}{\left(1-a b_{3} q / b_{4} b_{r+1} b_{r+2}\right)} \frac{\left(a b_{3} q / b_{4} b_{r+1} b_{r+2}, a q / b_{r+1} b_{r+2}, a q / b_{4} b_{r+1} ; q\right)_{k}}{\left(q, b_{3} q / b_{4}, b_{3} q / b_{r+2} ; q\right)_{k}} \\
& \times \frac{\left(a q / b_{4} b_{r+2}, b_{3} b_{5} / a, \ldots, b_{3} b_{r} / a, b_{3} b_{r+3} / a, \ldots, b_{3} b_{2 r} / a ; q\right)_{k}}{\left(b_{3} q / b_{r+1}, b_{3} q / b_{5}, \ldots, b_{3} q / b_{r}, b_{3} q / b_{r+3}, \ldots, b_{3} q / b_{2 r} ; q\right)_{k}} \\
& \times \frac{\left(b_{3}^{2} q / a ; q\right)_{2 k}}{\left(a b_{3} q^{2} / b_{4} b_{r+1} b_{r+2} ; q\right)_{2 k}}\left(\frac{a^{r-3} q^{r-3}}{b_{5} \ldots b_{r} b_{r+3} \ldots b_{2 r}}\right)^{k} \\
& \times \sum_{n=0}^{\infty} \frac{\left(1-b_{3}^{2} q^{2 k+2 n} / a\right)}{\left(1-b_{3}^{2} q^{2 k} / a\right)} \frac{\left(b_{3}^{2} q^{2 k} / a, b_{3} b_{5} q^{k} / a, \ldots, b_{3} b_{r} q^{k} / a ; q\right)_{n}}{\left(q, b_{3} q^{1+k} / b_{5}, \ldots, b_{3} q^{1+k} / b_{r} ; q\right)_{n}} \\
& \times \frac{\left(b_{3} b_{r+3} q^{k} / a, \ldots, b_{3} b_{2 r} q^{k} / a, b_{3} b_{4} b_{r+1} b_{r+2} / a^{2} q ; q\right)_{n}}{\left(b_{3} q^{1+k} / b_{r+3}, \ldots, b_{3} q^{1+k} / b_{2 r}, a b_{3} q^{2+2 k} / b_{4} b_{r+1} b_{r+2} ; q\right)_{n}}\left(\frac{a^{r-1} q^{r-2}}{b_{3} \ldots b_{2 r}}\right)^{n} \\
& +\left(\frac{\left(b_{3} q / b_{r+2}, b_{3} q / b_{r+1}, a q / b_{3} b_{r+1}, b_{4}, b_{4} / a, a q / b_{3} b_{r+2}, q, a, q / a ; q\right)_{\infty}}{\left(a b_{3} q^{2} / b_{4} b_{r+1} b_{r+2}, b_{4} / b_{3}, q / b_{r+2}, a q / b_{r+2}, b_{3} b_{4} / a, a^{2} q^{2} / b_{3} b_{4} b_{r+1} b_{r+2} ; q\right)_{\infty}}\right. \\
& \times \frac{\left(b_{3}, b_{6}, \ldots, b_{r}, b_{3} / a, b_{6} / a, \ldots, b_{r} / a, b_{5} q / b_{r+3}, \ldots, b_{5} q / b_{2 r} ; q\right)_{\infty}}{\left(q / b_{r+1}, a q / b_{r+1}, q / b_{5}, q / b_{r+3}, \ldots, q / b_{2 r}, a q / b_{5}, a q / b_{r+3}, \ldots, a q / b_{2 r} ; q\right)_{\infty}} \\
& \times \frac{\left(a b_{5} q^{2} / b_{4} b_{r+1} b_{r+2}, a q / b_{5} b_{r+3}, \ldots, a q / b_{5} b_{2 r}, a^{2} q^{2} / b_{4} b_{5} b_{r+1} b_{r+2} ; q\right)_{\infty}}{\left(b_{3} / b_{5}, b_{6} / b_{5}, \ldots, b_{r} / b_{5}, b_{3} b_{5} / a, b_{5} b_{6} / a, \ldots, b_{5} b_{r} / a, b_{5}^{2} q / a ; q\right)_{\infty}} \\
& \times \sum_{k=0}^{\infty} \frac{\left(1-a b_{3} q^{1+2 k} / b_{4} b_{r+1} b_{r+2}\right)}{\left(1-a b_{3} q / b_{4} b_{r+1} b_{r+2}\right)} \frac{\left(a b_{3} q / b_{4} b_{r+1} b_{r+2}, a q / b_{r+1} b_{r+2}, a q / b_{4} b_{r+1} ; q\right)_{k}}{\left(q, b_{3} q / b_{4}, b_{3} q / b_{r+2} ; q\right)_{k}} \\
& \times \frac{\left(a q / b_{4} b_{r+2}, b_{3} / b_{5}, b_{3} b_{5} / a ; q\right)_{k}}{\left(b_{3} q / b_{r+1}, a b_{5} q^{2} / b_{4} b_{r+1} b_{r+2}, a^{2} q^{2} / b_{4} b_{5} b_{r+1} b_{r+2} ; q\right)_{k}} q^{k} \\
& \times \sum_{n=0}^{\infty} \frac{\left(1-b_{5}^{2} q^{2 n} / a\right)}{\left(1-b_{5}^{2} / a\right)} \frac{\left(b_{5}^{2} / a, b_{3} b_{5} q^{k} / a, b_{5} b_{6} / a, \ldots, b_{5} b_{r} / a ; q\right)_{n}}{\left(q, b_{5} q^{1-k} / b_{3}, b_{5} q / b_{6}, \ldots, b_{5} q / b_{r} ; q\right)_{n}} \\
& \times \frac{\left(b_{5} b_{r+3} / a, \ldots, b_{5} b_{2 r} / a, b_{4} b_{5} b_{r+1} b_{r+2} q^{-1-k} / a^{2} ; q\right)_{n}}{\left(b_{5} q / b_{r+3}, \ldots, b_{5} q / b_{2 r}, a b_{5} q^{2+k} / b_{4} b_{r+1} b_{r+2} ; q\right)_{n}}\left(\frac{a^{r-1} q^{r-2}}{b_{3} \ldots b_{2 r}}\right)^{n} \\
& \left.\left.+\operatorname{idem}\left(b_{5} ; b_{6}, \ldots, b_{r}\right)\right)\right]+\operatorname{idem}\left(b_{3} ; b_{4}\right) \text {. }
\end{aligned}
$$

We have in (6.4) a sum of $2(r-3)$ double sums. Accordingly, for more clarity, let us write the whole expression in (6.4) as

$$
\begin{array}{r}
T_{1}\left(b_{3}, b_{4}\right)+T_{2}\left(b_{3}, b_{4}\right)+\cdots+T_{r-3}\left(b_{3}, b_{4}\right) \\
+U_{1}\left(b_{3}, b_{4}\right)+U_{2}\left(b_{3}, b_{4}\right)+\cdots+U_{r-3}\left(b_{3}, b_{4}\right),
\end{array}
$$


where, by definition of "idem", $U_{i}\left(b_{3}, b_{4}\right)=T_{i}\left(b_{4}, b_{3}\right)$ for $i=1, \ldots, r-3$. Further, $\sum_{i=2}^{r-3} T_{i}\left(b_{3}, b_{4}\right)=T_{2}\left(b_{3}, b_{4}\right)+\operatorname{idem}\left(b_{5} ; b_{6}, \ldots, b_{r}\right)\left(\right.$ and $\sum_{i=2}^{r-3} U_{i}\left(b_{3}, b_{4}\right)=$ $\left.U_{2}\left(b_{3}, b_{4}\right)+\operatorname{idem}\left(b_{5} ; b_{6}, \ldots, b_{r}\right)\right)$. Below, we will selectively perform manipulations with the respective terms $T_{i}$ and $U_{i}(i=1, \ldots, r-3)$.

To evaluate $T_{1}\left(b_{3}, b_{4}\right)$ (and hence also $U_{1}\left(b_{3}, b_{4}\right)$ ), we first shift the index $n$ of the inner sum in $T_{1}\left(b_{3}, b_{4}\right)$ (or, equivalently, in the first term in (6.4) ) by $-k$ and then interchange the double sum. Symbolically, we apply the interchange of summations as in (5.5). Thus, we obtain (using some elementary identities for $q$-shifted factorials)

$$
\begin{gathered}
T_{1}\left(b_{3}, b_{4}\right)=\frac{\left(q, a, q / a, b_{4}, \ldots, b_{r}, b_{4} / a, \ldots, b_{r} / a ; q\right)_{\infty}}{\left(q / b_{3}, q / b_{r+1}, \ldots, q / b_{2 r}, a q / b_{3}, a q / b_{r+1}, \ldots, a q / b_{2 r} ; q\right)_{\infty}} \\
\times \frac{\left(b_{3} q / b_{r+1}, \ldots, b_{3} q / b_{2 r}, a q / b_{3} b_{r+1}, \ldots, a q / b_{3} b_{2 r} ; q\right)_{\infty}}{\left(b_{4} / b_{3}, \ldots, b_{r} / b_{3}, b_{3} b_{4} / a, \ldots, b_{3} b_{r} / a, b_{3}^{2} q / a ; q\right)_{\infty}} \\
\times \sum_{n=0}^{\infty} \frac{\left(1-b_{3}^{2} q^{2 n} / a\right)}{\left(1-b_{3}^{2} / a\right)} \frac{\left(b_{3}^{2} / a, b_{3} b_{5} / a, \ldots, b_{3} b_{r} / a ; q\right)_{n}}{\left(q, b_{3} q / b_{5}, \ldots, b_{3} q / b_{r} ; q\right)_{n}} \\
\times \frac{\left(b_{3} b_{r+3} / a, \ldots, b_{3} b_{2 r} / a, b_{3} b_{4} b_{r+1} b_{r+2} / a^{2} q ; q\right)_{n}}{\left(b_{3} q / b_{r+3}, \ldots, b_{3} q / b_{2 r}, a b_{3} q^{2} / b_{4} b_{r+1} b_{r+2} ; q\right)_{n}}\left(\frac{a^{r-1} q_{3} \ldots b_{2 r}}{b_{3}}\right)^{n} \\
\times \sum_{k=0}^{n} \frac{\left(1-a b_{3} q^{1+2 k} / b_{4} b_{r+1} b_{r+2}\right)}{\left(1-a b_{3} q / b_{4} b_{r+1} b_{r+2}\right)} \frac{\left(a b_{3} q / b_{4} b_{r+1} b_{r+2}, a q / b_{r+1} b_{r+2}, a q / b_{4} b_{r+1} ; q\right)_{k}}{\left(q, b_{3} q / b_{4}, b_{3} q / b_{r+2} ; q\right)_{k}} \\
\times \frac{\left(a q / b_{4} b_{r+2}, b_{3}^{2} q^{n} / a, q^{-n} ; q\right)_{k}}{\left(b_{3} q / b_{r+1}, a^{2} q^{2-n} / b_{3} b_{4} b_{r+1} b_{r+2}, a b_{3} q^{2+n} / b_{4} b_{r+1} b_{r+2} ; q\right)_{k}} q^{k} .
\end{gathered}
$$

Now the inner sum can be evaluated by Jackson's terminating ${ }_{8} \phi_{7}$ summation in (3.6), which simplifies the last expression, $T_{1}\left(b_{3}, b_{4}\right)$, to

$$
\begin{aligned}
& \frac{\left(q, a, q / a, b_{4}, \ldots, b_{r}, b_{4} / a, \ldots, b_{r} / a ; q\right)_{\infty}}{\left(q / b_{3}, q / b_{r+1}, \ldots, q / b_{2 r}, a q / b_{3}, a q / b_{r+1}, \ldots, a q / b_{2 r} ; q\right)_{\infty}} \\
& \times \frac{\left(b_{3} q / b_{r+1}, \ldots, b_{3} q / b_{2 r}, a q / b_{3} b_{r+1}, \ldots, a q / b_{3} b_{2 r} ; q\right)_{\infty}}{\left(b_{4} / b_{3}, \ldots, b_{r} / b_{3}, b_{3} b_{4} / a, \ldots, b_{3} b_{r} / a, b_{3}^{2} q / a ; q\right)_{\infty}} \\
& \times \sum_{n=0}^{\infty} \frac{\left(1-b_{3}^{2} q^{2 n} / a\right)}{\left(1-b_{3}^{2} / a\right)} \frac{\left(b_{3}^{2} / a, b_{3} b_{4} / a, \ldots, b_{3} b_{2 r} / a ; q\right)_{n}}{\left(q, b_{3} q / b_{4}, \ldots, b_{3} q / b_{2 r} ; q\right)_{n}}\left(\frac{a^{r-1} q^{r-2}}{b_{3} \ldots b_{2 r}}\right)^{n} .
\end{aligned}
$$

Next, we consider $T_{2}\left(b_{3}, b_{4}\right)$ and $U_{2}\left(b_{3}, b_{4}\right)$. By interchanging the double sums in $T_{2}\left(b_{3}, b_{4}\right)$ and in $U_{2}\left(b_{3}, b_{4}\right)$ we obtain

$$
\begin{aligned}
& T_{2}\left(b_{3}, b_{4}\right)+U_{2}\left(b_{3}, b_{4}\right) \\
& =\frac{\left(b_{3} q / b_{r+2}, b_{3} q / b_{r+1}, a q / b_{3} b_{r+1}, b_{4}, b_{4} / a, a q / b_{3} b_{r+2}, q, a, q / a ; q\right)_{\infty}}{\left(a b_{3} q^{2} / b_{4} b_{r+1} b_{r+2}, b_{4} / b_{3}, q / b_{r+2}, a q / b_{r+2}, b_{3} b_{4} / a, a^{2} q^{2} / b_{3} b_{4} b_{r+1} b_{r+2} ; q\right)_{\infty}} \\
& \times \frac{\left(b_{3}, b_{6}, \ldots, b_{r}, b_{3} / a, b_{6} / a, \ldots, b_{r} / a, b_{5} q / b_{r+3}, \ldots, b_{5} q / b_{2 r} ; q\right)_{\infty}}{\left(q / b_{r+1}, a q / b_{r+1}, q / b_{5}, q / b_{r+3}, \ldots, q / b_{2 r}, a q / b_{5}, a q / b_{r+3}, \ldots, a q / b_{2 r} ; q\right)_{\infty}} \\
& \quad \times \frac{\left(a b_{5} q^{2} / b_{4} b_{r+1} b_{r+2}, a q / b_{5} b_{r+3}, \ldots, a q / b_{5} b_{2 r}, a^{2} q^{2} / b_{4} b_{5} b_{r+1} b_{r+2} ; q\right)_{\infty}}{\left(b_{3} / b_{5}, b_{6} / b_{5}, \ldots, b_{r} / b_{5}, b_{3} b_{5} / a, b_{5} b_{6} / a, \ldots, b_{5} b_{r} / a, b_{5}^{2} q / a ; q\right)_{\infty}} \\
& \quad \times \sum_{n=0}^{\infty} \frac{\left(1-b_{5}^{2} q^{2 n} / a\right)}{\left(1-b_{5}^{2} / a\right)} \frac{\left(b_{5}^{2} / a, b_{3} b_{5} / a, b_{5} b_{6} / a, \ldots, b_{5} b_{r} / a ; q\right)_{n}}{\left(q, b_{5} q / b_{3}, b_{5} q / b_{6}, \ldots, b_{5} q / b_{r} ; q\right)_{n}}
\end{aligned}
$$




$$
\begin{gathered}
\times \frac{\left(b_{5} b_{r+3} / a, \ldots, b_{5} b_{2 r} / a, b_{4} b_{5} b_{r+1} b_{r+2} / a^{2} q ; q\right)_{n}}{\left(b_{5} q / b_{r+3}, \ldots, b_{5} q / b_{2 r}, a b_{5} q^{2} / b_{4} b_{r+1} b_{r+2} ; q\right)_{n}}\left(\frac{a^{r-1} q^{r-2}}{b_{3} \ldots b_{2 r}}\right)^{n} \\
\times \sum_{k=0}^{\infty} \frac{\left(1-a b_{3} q^{1+2 k} / b_{4} b_{r+1} b_{r+2}\right)}{\left(1-a b_{3} q / b_{4} b_{r+1} b_{r+2}\right)} \frac{\left(a b_{3} q / b_{4} b_{r+1} b_{r+2}, a q / b_{r+1} b_{r+2}, a q / b_{4} b_{r+1} ; q\right)_{k}}{\left(q, b_{3} q / b_{4}, b_{3} q / b_{r+2} ; q\right)_{k}} \\
\times \frac{\left(a q / b_{4} b_{r+2}, b_{3} q^{-n} / b_{5}, b_{3} b_{5} q^{n} / a ; q\right)_{k}}{\left(b_{3} q / b_{r+1}, a b_{5} q^{2+n} / b_{4} b_{r+1} b_{r+2}, a^{2} q^{2-n} / b_{4} b_{5} b_{r+1} b_{r+2} ; q\right)_{k}} q^{k} \\
+\operatorname{idem}\left(b_{3} ; b_{4}\right) .
\end{gathered}
$$

Now, to the inner sum of the first double sum (but not of the second!) in (6.7) we apply Bailey's nonterminating ${ }_{8} \phi_{7}$ summation (4.2), i.e., specifically we apply

$$
\begin{aligned}
\sum_{k=0}^{\infty} \frac{\left(1-a b_{3} q^{1+2 k} / b_{4} b_{r+1} b_{r+2}\right)}{\left(\left(1-a b_{3} q / b_{4} b_{r+1} b_{r+2}\right)\right)} \frac{\left(a b_{3} q / b_{4} b_{r+1} b_{r+2}, a q / b_{r+1} b_{r+2} ; q\right)_{k}}{\left(q, b_{3} q / b_{4}, b_{3} q / b_{r+2} ; q\right)_{k}} \\
\quad \times \frac{\left(a q / b_{4} b_{r+1}, a q / b_{4} b_{r+2}, b_{3} q^{-n} / b_{5}, b_{3} b_{5} q^{n} / a ; q\right)_{k}}{\left(b_{3} q / b_{r+1}, a b_{5} q^{2+n} / b_{4} b_{r+1} b_{r+2}, a^{2} q^{2-n} / b_{4} b_{5} b_{r+1} b_{r+2} ; q\right)_{k}} q^{k} \\
=\frac{\left(a b_{3} q^{2} / b_{4} b_{r+1} b_{r+2}, b_{4} / b_{3}, b_{3} b_{4} / a, b_{5} q^{1+n} / b_{r+2} ; q\right)_{\infty}}{\left(b_{3} q / b_{r+2}, b_{3} q / b_{r+1}, a b_{5} q^{2+n} / b_{4} b_{r+1} b_{r+2}, a^{2} q^{2-n} / b_{4} b_{5} b_{r+1} b_{r+2} ; q\right)_{\infty}} \\
\quad \times \frac{\left(a q^{1-n} / b_{5} b_{r+2}, b_{5} q^{1+n} / b_{r+1}, a q^{1-n} / b_{5} b_{r+1}, a^{2} q^{2} / b_{3} b_{4} b_{r+1} b_{r+2} ; q\right)_{\infty}}{\left(a q / b_{3} b_{r+1}, a q / b_{3} b_{r+2}, b_{4} q^{-n} / b_{5}, b_{4} b_{5} q^{n} / a ; q\right)_{\infty}} \\
-\frac{\left(a b_{3} q^{2} / b_{4} b_{r+1} b_{r+2}, a q / b_{4} b_{r+1}, a q / b_{4} b_{r+2}, b_{3} q^{-n} / b_{5}, b_{3} b_{5} q^{n} / a ; q\right)_{\infty}}{\left(b_{3} / b_{4}, b_{3} q / b_{r+2}, b_{3} q / b_{r+1}, a b_{5} q^{2+n} / b_{4} b_{r+1} b_{r+2}, a^{2} q^{2-n} / b_{4} b_{5} b_{r+1} b_{r+2} ; q\right)_{\infty}} \\
\times \frac{\left(b_{4} / b_{3}, b_{4} q / b_{r+2}, b_{4} q / b_{r+1}, a b_{5} q^{2+n} / b_{3} b_{r+1} b_{r+2}, a^{2} q^{2-n} / b_{3} b_{5} b_{r+1} b_{r+2} ; q\right)_{\infty}}{\left(a q / b_{3} b_{r+1}, a q / b_{3} b_{r+2}, b_{4} q^{-n} / b_{5}, b_{4} b_{5} q^{n} / a, a b_{4} q^{2} / b_{3} b_{r+1} b_{r+2} ; q\right)_{\infty}} \\
\quad \times \sum_{k=0}^{\infty} \frac{\left(1-a b_{4} q^{1+2 k} / b_{3} b_{r+1} b_{r+2}\right)}{\left(1-a b_{4} q / b_{3} b_{r+1} b_{r+2}\right)} \frac{\left(a b_{4} q / b_{3} b_{r+1} b_{r+2}, a q / b_{r+1} b_{r+2} ; q\right)_{k}}{\left(q, b_{4} q / b_{3} ; q\right)_{k}} \\
\times \frac{\left(a q / b_{3} b_{r+1}, a q / b_{3} b_{r+2}, b_{4} q^{-n} / b_{5}, b_{4} b_{5} q^{n} / a ; q\right)_{k}}{\left(b_{4} q / b_{r+2}, b_{4} q / b_{r+1}, a b_{5} q^{2+n} / b_{3} b_{r+1} b_{r+2}, a^{2} q^{2-n} / b_{3} b_{5} b_{r+1} b_{r+2} ; q\right)_{k}} q^{k} .
\end{aligned}
$$

The result of the application of this (two term) summation is that the first term in (6.7), $T_{2}\left(b_{3}, b_{4}\right)$, is split into two parts, one single sum $T_{2}^{\prime}\left(b_{3}, b_{4}\right)$ and one double sum $T_{2}^{\prime \prime}\left(b_{3}, b_{4}\right)$. Formally, we have

$$
T_{2}\left(b_{3}, b_{4}\right)+U_{2}\left(b_{3}, b_{4}\right)=\left[T_{2}^{\prime}\left(b_{3}, b_{4}\right)+T_{2}^{\prime \prime}\left(b_{3}, b_{4}\right)\right]+U_{2}\left(b_{3}, b_{4}\right) .
$$

But $T_{2}^{\prime \prime}\left(b_{3}, b_{4}\right)$ is precisely $-U_{2}\left(b_{3}, b_{4}\right)$ (as can be readily checked), so two terms cancel, thus

$$
T_{2}\left(b_{3}, b_{4}\right)+U_{2}\left(b_{3}, b_{4}\right)=T_{2}^{\prime}\left(b_{3}, b_{4}\right) .
$$

Now, the last expression, $T_{2}^{\prime}\left(b_{3}, b_{4}\right)$, can be simplified to

$$
\begin{gathered}
\frac{\left(q, a, q / a, b_{3}, b_{4}, b_{6}, \ldots, b_{r}, b_{3} / a, b_{4} / a, b_{6} / a, \ldots, b_{r} / a ; q\right)_{\infty}}{\left(q / b_{5}, q / b_{r+1}, \ldots, q / b_{2 r}, a q / b_{5}, a q / b_{r+1}, \ldots, a q / b_{2 r}, b_{3} / b_{5}, b_{4} / b_{5} ; q\right)_{\infty}} \\
\times \frac{\left(b_{5} q / b_{r+1}, \ldots, b_{5} q / b_{2 r}, a q / b_{5} b_{r+1}, \ldots, a q / b_{5} b_{2 r} ; q\right)_{\infty}}{\left(b_{6} / b_{5}, \ldots, b_{r} / b_{5}, b_{3} b_{5} / a, b_{4} b_{5} / a, b_{5} b_{6} / a, \ldots, b_{5} b_{r} / a, b_{5}^{2} q / a ; q\right)_{\infty}} \\
\times \sum_{n=0}^{\infty} \frac{\left(1-b_{5}^{2} q^{2 n} / a\right)}{\left(1-b_{5}^{2} / a\right)} \frac{\left(b_{5}^{2} / a, b_{3} b_{5} / a, b_{4} b_{5} / a ; q\right)_{n}}{\left(q, b_{5} q / b_{3}, b_{5} q / b_{4} ; q\right)_{n}}
\end{gathered}
$$




$$
\times \frac{\left(b_{5} b_{6} / a, \ldots, b_{5} b_{2 r} / a ; q\right)_{n}}{\left(b_{5} q / b_{6}, \ldots, b_{5} q / b_{2 r} ; q\right)_{n}}\left(\frac{a^{r-1} q^{r-2}}{b_{3} \ldots b_{2 r}}\right)^{n} .
$$

It is easy to see that (6.9) equals (6.6) where $b_{3}$ and $b_{5}$ are interchanged. Collecting all terms, according to (6.5), completes our derivation of the very-well-poised ${ }_{2 r} \psi_{2 r}$ transformation formula in (6.1).

We conclude this section considering Slater's well-poised ${ }_{2 r} \psi_{2 r}$ transformation in its general form.

If, in (6.1), we set $b_{2 r-1}=-b_{2 r}=\sqrt{a}$, and then let $r \mapsto r+2$, and relabel $b_{i} \mapsto b_{i-2}$, we obtain the following transformation formula for a well-poised ${ }_{2 r} \psi_{2 r}$ series:

$$
\begin{aligned}
& { }_{2 r} \psi_{2 r}\left[\begin{array}{l}
b_{1}, b_{2}, \ldots, b_{2 r} \\
\frac{a q}{b_{1}}, \frac{a q}{b_{2}}, \ldots, \frac{a q}{b_{2 r}} ; q,-\frac{a^{r} q^{r}}{b_{1} \ldots b_{2 r}}
\end{array}\right] \\
& =\frac{\left(q, a, \frac{q}{a}, b_{2}, \ldots, b_{r}, \frac{b_{2}}{a}, \ldots, \frac{b_{r}}{a}, \frac{b_{1} q}{b_{r+1}}, \ldots, \frac{b_{1} q}{b_{2 r}}, \frac{a q}{b_{1} b_{r+1}}, \ldots, \frac{a q}{b_{1} b_{2 r}}, ; q\right)_{\infty}}{\left(\frac{q}{b_{1}}, \frac{q}{b_{r+1}}, \ldots, \frac{q}{b_{2 r}}, \frac{a q}{b_{1}}, \frac{a q}{b_{r+1}}, \ldots, \frac{a q}{b_{2 r}}, \frac{b_{2}}{b_{1}}, \ldots, \frac{b_{r}}{b_{1}}, \frac{b_{1} b_{2}}{a}, \ldots, \frac{b_{1} b_{r}}{a}, \frac{b_{1}^{2} q}{a} ; q\right)_{\infty}}
\end{aligned}
$$

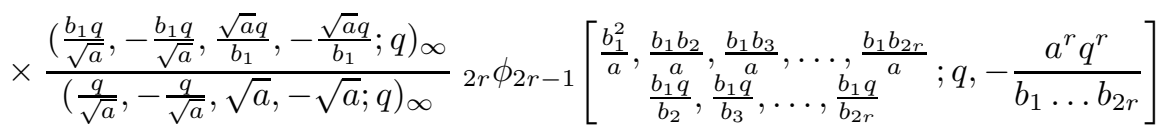

$$
\begin{aligned}
& +\operatorname{idem}\left(b_{1} ; b_{2}, \ldots, b_{r}\right),
\end{aligned}
$$

where the series either terminate, or $\left|a^{r} q^{r} / b_{1} \ldots b_{2 r}\right|<1$, for convergence. On the other hand, we can derive (6.1) from (6.10) by choosing $b_{2 r-1}=-b_{2 r}=q \sqrt{a}$ and relabelling of the parameters $b_{i} \mapsto b_{i+2}$. Thus, the transformations (6.1) and (6.10) are equivalent.

Slater [33], in fact, derived the more general transformation

$$
\begin{aligned}
& { }_{2 r} \psi_{2 r}\left[\begin{array}{l}
b_{1}, b_{2}, \ldots, b_{2 r} \\
\frac{a q}{b_{1}}, \frac{a q}{b_{2}}, \ldots, \frac{a q}{b_{2 r}} ; q,-\frac{a^{r} q^{r}}{b_{1} \ldots b_{2 r}}
\end{array}\right] \\
& =\frac{\left(a, \frac{q}{a}, a_{2}, \ldots, a_{r}, \frac{q}{a_{2}}, \ldots, \frac{q}{a_{r}}, \frac{a_{2}}{a}, \ldots, \frac{a_{r}}{a}, \frac{a q}{a_{2}}, \ldots, \frac{a q}{a_{r}} ; q\right)_{\infty}}{\left(\frac{q}{b_{1}}, \ldots, \frac{q}{b_{2 r}}, \frac{a q}{b_{1}}, \ldots, \frac{a q}{b_{2 r}}, \frac{a_{2}}{a_{1}}, \ldots, \frac{a_{r}}{a_{1}}, \frac{a_{1} q}{a_{2}}, \ldots, \frac{a_{1} q}{a_{r}} ; q\right)_{\infty}} \\
& \times \frac{\left(\frac{a_{1} q}{b_{1}}, \ldots, \frac{a_{1} q}{b_{2 r}}, \frac{a q}{a_{1} b_{1}}, \ldots, \frac{a q}{a_{1} b_{2 r}}, \frac{a_{1}}{\sqrt{a}},-\frac{a_{1}}{\sqrt{a}}, \frac{\sqrt{a} q}{a_{1}},-\frac{\sqrt{a} q}{a_{1}} ; q\right)_{\infty}}{\left(\frac{a_{1} a_{2}}{a}, \ldots, \frac{a_{1} a_{r}}{a}, \frac{a q}{a_{1} a_{2}}, \ldots, \frac{a q}{a_{1} a_{r}}, \frac{a_{1}^{2}}{a}, \frac{a q}{a_{1}^{2}}, \frac{q}{\sqrt{a}},-\frac{q}{\sqrt{a}}, \sqrt{a},-\sqrt{a} ; q\right)_{\infty}} \\
& \times{ }_{2 r} \psi_{2 r}\left[\frac{\frac{a_{1} b_{1}}{a}, \frac{a_{1} b_{2}}{a_{a}}, \ldots, \frac{a_{1} b_{2 r}}{a} ; q,-\frac{a^{r} q^{r}}{b_{1} q} b_{1} \ldots b_{2 r}}{\frac{a_{1} q}{b_{2}}}, \ldots, \frac{a_{1} q}{b_{2 r}} ; \operatorname{idem}\left(a_{1} ; a_{2}, \ldots, a_{r}\right),\right.
\end{aligned}
$$

where the series either terminate, or $\left|a^{r} q^{r} / b_{1} \ldots b_{2 r}\right|<1$, for convergence.

It is easy to see, that the transformation in (6.11) which involves only bilateral series is much more general than the transformation in (6.10) since in (6.11) we have $r$ additional parameters $a_{1}, \ldots, a_{r}$. The special case $a_{i}=b_{i}, i=1, \ldots, r$, of (6.11) is exactly (6.10).

We were able to prove the transformation in (6.10) (which is equivalent to (6.1)) by induction. We started from less complicated identities and ultimatively deduced more complex ones involving more parameters. The natural question arises whether we can also prove the more general transformation (6.11) by elementary means. Unfortunately, we were not able to derive (6.11) directly by the method of this article. Instead, we want to point out that the transformation we derived, (6.10), 
can be extended to (6.11) by an $r$-fold application of "Ismail's [20] argument" (which is actually a classical analytic continuation argument). We provide a sketch of how this works.

It is easy to see that both sides of (6.11) are analytic in each $b_{1}^{-1}, b_{2}^{-1}, \ldots, b_{r}^{-1}$ in a domain around the origin. We know that the identity is true when $b_{i}=a_{i}$, for $i=1, \ldots, r$. What needs to be done is to extend (6.10) first by an additional parameter $b_{1}$, then by $b_{2}$, etc. This means that if we have already extended (6.10) by $b_{1}, \ldots, b_{j}$, what we should have derived is the identity in (6.11) where $b_{i}=a_{i}$, for $i=j+1, \ldots, r$. So, we proceed by induction starting with $j=0$ (identity (6.10)) and ending with $j=r$ (identity (6.11)). In the inductive step, we consider the transformation (6.11) where $b_{i}=a_{i}$, for $i=j+1, \ldots, r$. We call that identity $E_{j+1}$. We need to show that $E_{j+1}$ is true, provided $E_{j}$ is true, which is (6.11) where $b_{i}=a_{i}$, for $i=j, \ldots, r$. We observe that both sides of $E_{j+1}$ are analytic in $1 / b_{j}$ around the origin. The identity is true for $b_{j}=a_{j} q^{-n_{j}}, n_{j}=0,1,2, \ldots$ This follows by the $a_{j} \mapsto a_{j} q^{-n_{j}}$ case of the inductive hypothesis, $E_{j}$. (This can be verified by looking at all the terms involving $j$. Further note that the index of the $j$-th sum is shifted by $-n_{j}$, since the $j$-th bilateral series becomes a unilateral series.) Since $E_{j+1}$ is true for an infinite sequence of $1 / b_{j}$ which has an accumulation point, namely 0 , in the interior of the domain $\mathcal{D}$ of analyticity of $1 / b_{j}$, we can apply the identity theorem to deduce that $E_{j+1}$ is true for $1 / b_{j}$ throughout $\mathcal{D}$. Now, by induction, (6.11) follows, with the general additional parameters $b_{1}, \ldots, b_{r}$.

\section{Slater's GENERAL BILATERAL TRANSFORMATIONS}

Here we consider series which are not necessarily well-poised. An important transformation for general ${ }_{r} \psi_{r}$ series was given by Slater [33]. Her formula in its general form, see (7.16), connects $r+1$ bilateral ${ }_{r} \psi_{r}$ series. However, the special case of the transformation where only one of the series is bilateral and the $r$ other series are all unilateral $\left({ }_{r} \phi_{r-1}\right.$ series $)$ itself is interesting, as it includes many important identities for (bilateral) basic hypergeometric series as special cases. This formula reads as follows:

$$
\begin{aligned}
& { }_{r} \psi_{r}\left[\begin{array}{c}
a_{1}, a_{2}, \ldots, a_{r} \\
b_{1}, b_{2}, \ldots, b_{r}
\end{array} ; q, z\right] \\
& =\frac{\left(q, a_{2}, \ldots, a_{r}, \frac{b_{1}}{a_{1}}, \ldots, \frac{b_{r}}{a_{1}}, a_{1} z, \frac{q}{a_{1} z} ; q\right)_{\infty}}{\left(\frac{q}{a_{1}}, \frac{a_{2}}{a_{1}}, \ldots, \frac{a_{r}}{a_{1}}, b_{1}, \ldots, b_{r}, z, \frac{q}{z} ; q\right)_{\infty}} \phi_{r-1}\left[\begin{array}{c}
\frac{a_{1} q}{b_{1}}, \frac{a_{1} q}{b_{2}}, \ldots, \frac{a_{1} q}{b_{r}} \\
\frac{a_{1} q}{a_{2}}, \ldots, \frac{a_{1} q}{a_{r}}
\end{array} ; q, \frac{b_{1} \ldots b_{r}}{a_{1} \ldots a_{r} z}\right] \\
& +\operatorname{idem}\left(a_{1} ; a_{2}, \ldots, a_{r}\right) \text {, }
\end{aligned}
$$

where the series either terminate, or $\left|b_{1} \ldots b_{r} / a_{1} \ldots a_{r}\right|<|z|<1$, for convergence. (The symbol "idem $\left(a_{1} ; a_{2}, \ldots, a_{r}\right)$ " is explained in the introduction.)

We are able to give an elementary inductive proof of this general transformation formula. We proceed by similar means as in the previous sections where Slater's general transformation for well-poised bilateral ${ }_{2 r} \psi_{2 r}$ series was derived. For deriving the transformation in (7.1) we make use of other identities. In particular, where we were before using identities for very-well-poised ${ }_{8} \phi_{7}$ series, here we instead utilize identities for balanced ${ }_{3} \phi_{2}$ series.

The $r=1$ case of $(7.1)$ is readily verifed using the ${ }_{1} \psi_{1}$ and ${ }_{1} \phi_{0}$ summations in (7.2) and in (7.3), respectively. Ramanujan's 18] ${ }_{1} \psi_{1}$ summation (cf. [15. 
Eq. (5.2.1)]) reads

$$
{ }_{1} \psi_{1}\left[\begin{array}{l}
a \\
b
\end{array} ; q, z\right]=\frac{(q, b / a, a z, q / a z ; q)_{\infty}}{(b, q / a, z, b / a z ; q)_{\infty}}
$$

where the series either terminates, or $|b / a|<|z|<1$, for convergence. The summation in (7.2) is a bilateral extension of the $q$-binomial theorem (cf. [15, Sec. 1.3]),

$$
{ }_{1} \phi_{0}\left[{ }_{-}^{a} ; q, z\right]=\frac{(a z ; q)_{\infty}}{(z ; q)_{\infty}}
$$

where the series either terminates, or $|z|<1$, for convergence. The summation in (7.3) was first discovered by Cauchy [9]. Clearly, (7.2) reduces to (7.3) when $b=q$. The first elementary proof of the ${ }_{1} \psi_{1}$ summation formula (7.2) was given by M. Jackson (as pointed out to us by George Andrews [3]). Jackson's proof essentially derives the ${ }_{1} \psi_{1}$ summation from the $q$-Gauß summation, by manipulation of series. In 30] we reviewed Jackson's proof and extended it to a method to provide new elementary proofs of Dougall's $13 .{ }_{2} H_{2}$ summation and Bailey's $[7$ very-well-poised ${ }_{6} \psi_{6}$ summation, respectively.

Now let us establish the $r=2$ case of (7.1).

Here, and later we make use of a special case of the identity in (4.3). Namely set $b \mapsto a_{2}, c \mapsto a q / b_{2}, d \mapsto a_{1}$, and $e \mapsto a q / b_{1}$ in (4.3), and then let $a \rightarrow \infty$. We obtain

$$
\begin{array}{r}
\frac{\left(b_{1} b_{2} / a_{2}, b_{2} / a_{1}, a_{2}, b_{1} / a_{1} ; q\right)_{\infty}}{\left(a_{2} / a_{1}, b_{1}, b_{1} b_{2} / a_{1} a_{2}, b_{2} ; q\right)_{\infty}} \sum_{k=0}^{\infty} \frac{\left(b_{1} / a_{2}, b_{2} / a_{2} ; q\right)_{k}\left(a_{1} ; q\right)_{n+k}}{\left(q, a_{1} q / a_{2} ; q\right)_{k}\left(b_{1} b_{2} / a_{2} ; q\right)_{n+k}} q^{k} \\
+\operatorname{idem}\left(a_{1} ; a_{2}\right)=\frac{\left(a_{1}, a_{2} ; q\right)_{n}}{\left(b_{1}, b_{2} ; q\right)_{n}} .
\end{array}
$$

Alternatively, we could also have invoked the non-terminating $q$-Pfaff-Saalschütz summation theorem (cf. 15, Eq. (II.24)]),

$$
\begin{array}{r}
{ }_{3} \phi_{2}\left[\begin{array}{c}
a, b, c \\
e, f
\end{array} ; q, q\right]+\frac{(q / e, a, b, c, f q / e ; q)_{\infty}}{(e / q, a q / e, b q / e, c q / e, f ; q)_{\infty}}{ }_{3} \phi_{2}\left[\begin{array}{c}
a q / e, b q / e, c q / e \\
q^{2} / e, f q / e
\end{array} ; q, q\right] \\
=\frac{(q / e, f / a, f / b, f / c ; q)_{\infty}}{(a q / e, b q / e, c q / e, f ; q)_{\infty}}
\end{array}
$$

where $e f=a b c q$. If we simultaneously replace $a, b, c$, and $e$ in $(7.5)$ by $b_{1} / a_{2}$, $b_{2} / a_{2}, a_{1} q^{n}$, and $a_{1} q / a_{2}$, respectively, we obtain after some manipulations (7.4).

For the $r=2$ case of (7.1), we multiply both sides of (7.4) by $z^{n}$ and sum over all integers $n$. On the right side we obtain

$$
{ }_{2} \psi_{2}\left[\begin{array}{c}
a_{1}, a_{2} \\
b_{1}, b_{2}
\end{array} ;, z\right] .
$$

On the left side we obtain

$$
\begin{aligned}
& \frac{\left(b_{1} b_{2} / a_{2}, b_{2} / a_{1}, a_{2}, b_{1} / a_{1} ; q\right)_{\infty}}{\left(a_{2} / a_{1}, b_{1}, b_{1} b_{2} / a_{1} a_{2}, b_{2} ; q\right)_{\infty}} \sum_{n=-\infty}^{\infty} z^{n} \sum_{k=0}^{\infty} \frac{\left(b_{1} / a_{2}, b_{2} / a_{2} ; q\right)_{k}\left(a_{1} ; q\right)_{n+k}}{\left(q, a_{1} q / a_{2} ; q\right)_{k}\left(b_{1} b_{2} / a_{2} ; q\right)_{n+k}} q^{k} \\
& +\operatorname{idem}\left(a_{1} ; a_{2}\right)=\frac{\left(b_{1} b_{2} / a_{2}, b_{2} / a_{1}, a_{2}, b_{1} / a_{1} ; q\right)_{\infty}}{\left(a_{2} / a_{1}, b_{1}, b_{1} b_{2} / a_{1} a_{2}, b_{2} ; q\right)_{\infty}} \sum_{k=0}^{\infty} \frac{\left(b_{1} / a_{2}, b_{2} / a_{2}, a_{1} ; q\right)_{k}}{\left(q, a_{1} q / a_{2}, b_{1} b_{2} / a_{2} ; q\right)} q^{k}
\end{aligned}
$$




$$
\times \sum_{n=-\infty}^{\infty} \frac{\left(a_{1} q^{k} ; q\right)_{n}}{\left(b_{1} b_{2} q^{k} / a_{2} ; q\right)_{n}} z^{n}+\operatorname{idem}\left(a_{1} ; a_{2}\right) .
$$

Now we can evaluate the the inner sums by the ${ }_{1} \psi_{1}$ summation (7.2). This yields

$$
\begin{aligned}
\frac{\left(b_{1} b_{2} / a_{2}, b_{2} / a_{1}, a_{2}, b_{1} / a_{1} ; q\right)_{\infty}}{\left(a_{2} / a_{1}, b_{1}, b_{1} b_{2} / a_{1} a_{2}, b_{2} ; q\right)_{\infty}} \sum_{k=0}^{\infty} \frac{\left(b_{1} / a_{2}, b_{2} / a_{2}, a_{1} ; q\right)_{k}}{\left(q, a_{1} q / a_{2}, b_{1} b_{2} / a_{2} ; q\right)_{k}} q^{k} \\
\times \frac{\left(q, b_{1} b_{2} / a_{1} a_{2}, a_{1} z q^{k}, q^{1-k} / a_{1} z ; q\right)_{\infty}}{\left(b_{1} b_{2} q^{k} / a_{2}, q^{1-k} / a_{1}, z, b_{1} b_{2} / a_{1} a_{2} z ; q\right)_{\infty}}+\operatorname{idem}\left(a_{1} ; a_{2}\right),
\end{aligned}
$$

which can be simplified to

$$
\begin{aligned}
\frac{\left(b_{2} / a_{1}, a_{2}, b_{1} / a_{1}, q, a_{1} z, q / a_{1} z ; q\right)_{\infty}}{\left(a_{2} / a_{1}, b_{1}, b_{2}, q / a_{1}, z, b_{1} b_{2} / a_{1} a_{2} z ; q\right)_{\infty}} \sum_{k=0}^{\infty} \frac{\left(b_{1} / a_{2}, b_{2} / a_{2} ; q\right)_{k}}{\left(q, a_{1} q / a_{2} ; q\right)_{k}}\left(\frac{q}{z}\right)^{k} & +\operatorname{idem}\left(a_{1} ; a_{2}\right) .
\end{aligned}
$$

Now, to the ${ }_{2} \phi_{1}$ 's in (7.6) we apply Heine's [19] $q$-Euler transformation (see [15. Eq. (1.4.3)]):

$$
{ }_{2} \phi_{1}\left[\begin{array}{c}
a, b \\
c
\end{array} ; q, z\right]=\frac{(a b z / c ; q)_{\infty}}{(z ; q)_{\infty}}{ }_{2} \phi_{1}\left[\begin{array}{c}
c / a, c / b \\
c
\end{array} ;, \frac{a b z}{c}\right],
$$

where $\max (|z|,|a b z / c|)<1$. Hence, by $(7.7)$ the expression in $(7.6)$ is transformed into

$$
\frac{\left(b_{2} / a_{1}, a_{2}, b_{1} / a_{1}, q, a_{1} z, q / a_{1} z ; q\right)_{\infty}}{\left(a_{2} / a_{1}, b_{1}, b_{2}, q / a_{1}, z, q / z ; q\right)_{\infty}} \phi_{1}\left[\begin{array}{c}
a_{1} q / b_{1}, a_{1} q / b_{2} \\
a_{1} q / a_{2}
\end{array} ;, \frac{b_{1} b_{2}}{a_{1} a_{2} z}\right]+\operatorname{idem}\left(a_{1} ; a_{2}\right),
$$

which is exactly the right side of the $r=2$ case of (7.1).

Now suppose that the formula in (7.1) is already shown for any integer $t$ where $1 \leq t<r$. To show the transformation for $t=r$, we multiply both sides of (7.4) by

$$
\frac{\left(a_{3}, \ldots, a_{r} ; q\right)_{n}}{\left(b_{3}, \ldots, b_{r} ; q\right)_{n}} z^{n}
$$

and sum over all integers $n$.

On the right side we obtain

$$
{ }_{r} \psi_{r}\left[\begin{array}{c}
a_{1}, a_{2}, \ldots, a_{r} \\
b_{1}, b_{2}, \ldots, b_{r}
\end{array} ;, z\right] .
$$

On the left side we obtain

$$
\begin{gathered}
\frac{\left(b_{1} b_{2} / a_{2}, b_{2} / a_{1}, a_{2}, b_{1} / a_{1} ; q\right)_{\infty}}{\left(a_{2} / a_{1}, b_{1}, b_{1} b_{2} / a_{1} a_{2}, b_{2} ; q\right)_{\infty}} \sum_{n=-\infty}^{\infty} \frac{\left(a_{3}, \ldots, a_{r} ; q\right)_{n}}{\left(b_{3}, \ldots, b_{r} ; q\right)_{n}} z^{n} \\
\times \sum_{k=0}^{\infty} \frac{\left(b_{1} / a_{2}, b_{2} / a_{2} ; q\right)_{k}\left(a_{1} ; q\right)_{n+k}}{\left(q, a_{1} q / a_{2} ; q\right)_{k}\left(b_{1} b_{2} / a_{2} ; q\right)_{n+k}} q^{k}+\operatorname{idem}\left(a_{1} ; a_{2}\right) \\
=\frac{\left(b_{1} b_{2} / a_{2}, b_{2} / a_{1}, a_{2}, b_{1} / a_{1} ; q\right)_{\infty}}{\left(a_{2} / a_{1}, b_{1}, b_{1} b_{2} / a_{1} a_{2}, b_{2} ; q\right)_{\infty}} \sum_{k=0}^{\infty} \frac{\left(b_{1} / a_{2}, b_{2} / a_{2}, a_{1} ; q\right)_{k}}{\left(q, a_{1} q / a_{2}, b_{1} b_{2} / a_{2} ; q\right)_{k}} q^{k} \\
\times \sum_{n=-\infty}^{\infty} \frac{\left(a_{1} q^{k}, a_{3}, \ldots, a_{r} ; q\right)_{n}}{\left(b_{1} b_{2} q^{k} / a_{2}, b_{3}, \ldots, b_{r} ; q\right)_{n}} z^{n}+\operatorname{idem}\left(a_{1} ; a_{2}\right) .
\end{gathered}
$$


Now to the inner sums we apply the inductive hypothesis (i.e., the $r \mapsto r-1$ case of $(7.1)$ ), and we obtain for the last expression

$$
\begin{aligned}
& \frac{\left(b_{1} b_{2} / a_{2}, b_{2} / a_{1}, a_{2}, b_{1} / a_{1} ; q\right)_{\infty}}{\left(a_{2} / a_{1}, b_{1}, b_{1} b_{2} / a_{1} a_{2}, b_{2} ; q\right)_{\infty}} \sum_{k=0}^{\infty} \frac{\left(b_{1} / a_{2}, b_{2} / a_{2}, a_{1} ; q\right)_{k}}{\left(q, a_{1} q / a_{2}, b_{1} b_{2} / a_{2} ; q\right)_{k}} q^{k} \\
& \times\left[\frac{\left(q, a_{3}, \ldots, a_{r}, b_{1} b_{2} / a_{1} a_{2}, b_{3} q^{-k} / a_{1}, \ldots, b_{r} q^{-k} / a_{1}, a_{1} z q^{k}, q^{1-k} / a_{1} z ; q\right)_{\infty}}{\left(q^{1-k} / a_{1}, a_{3} q^{-k} / a_{1}, \ldots, a_{r} q^{-k} / a_{1}, b_{1} b_{2} q^{k} / a_{2}, b_{3}, \ldots, b_{r}, z, q / z ; q\right)_{\infty}}\right. \\
& \quad \times \sum_{n=0}^{\infty} \frac{\left(a_{1} a_{2} q / b_{1} b_{2}, a_{1} q^{1+k} / b_{3}, \ldots, a_{1} q^{1+k} / b_{r} ; q\right)_{n}}{\left(q, a_{1} q^{1+k} / a_{3}, \ldots, a_{1} q^{1+k} / a_{r} ; q\right)_{n}}\left(\frac{b_{1} \ldots b_{r}}{a_{1} \ldots a_{r} z}\right)^{n} \\
& +\left(\frac{\left(q, a_{1} q^{k}, a_{4}, \ldots, a_{r}, b_{1} b_{2} q^{k} / a_{2} a_{3}, b_{3} / a_{3}, \ldots, b_{r} / a_{3}, a_{3} z, q / a_{3} z ; q\right)_{\infty}}{\left(q / a_{3}, a_{1} q^{k} / a_{3}, a_{4} / a_{3}, \ldots, a_{r} / a_{3}, b_{1} b_{2} q^{k} / a_{2}, b_{3}, \ldots, b_{r}, z, q / z ; q\right)_{\infty}}\right. \\
& \quad \times \sum_{n=0}^{\infty} \frac{\left(a_{2} a_{3} q^{1-k} / b_{1} b_{2}, a_{3} q / b_{3}, \ldots, a_{3} q / b_{r} ; q\right)_{n}}{\left(q, a_{3} q^{1-k} / a_{1}, a_{3} q / a_{4}, \ldots, a_{3} q / a_{r} ; q\right)_{n}}\left(\frac{b_{1} \ldots b_{r}}{a_{1} \ldots a_{r} z}\right)^{n} \\
& \left.\left.\quad+\operatorname{idem}\left(a_{3} ; a_{4}, \ldots, a_{r}\right)\right)\right]+\operatorname{idem}\left(a_{1} ; a_{2}\right),
\end{aligned}
$$

which can be simplified to

$$
\begin{gathered}
{\left[\frac{\left(b_{2} / a_{1}, a_{2}, b_{1} / a_{1}, q, a_{3}, \ldots, a_{r}, b_{3} / a_{1}, \ldots, b_{r} / a_{1}, a_{1} z, q / a_{1} z ; q\right)_{\infty}}{\left(a_{2} / a_{1}, b_{1}, b_{2}, q / a_{1}, a_{3} / a_{1}, \ldots, a_{r} / a_{1}, b_{3}, \ldots, b_{r}, z, q / z ; q\right)_{\infty}}\right.} \\
\quad \times \sum_{k=0}^{\infty} \frac{\left(b_{1} / a_{2}, b_{2} / a_{2}, a_{1} q / b_{3}, \ldots, a_{1} q / b_{r} ; q\right)_{k}}{\left(q, a_{1} q / a_{2}, a_{1} q / a_{3}, \ldots, a_{1} q / a_{r} ; q\right)_{k}}\left(\frac{b_{3} \ldots b_{r} q}{a_{3} \ldots a_{r} z}\right)^{k} \\
\times \sum_{n=0}^{\infty} \frac{\left(a_{1} a_{2} q / b_{1} b_{2}, a_{1} q^{1+k} / b_{3}, \ldots, a_{1} q^{1+k} / b_{r} ; q\right)_{n}}{\left(q, a_{1} q^{1+k} / a_{3}, \ldots, a_{1} q^{1+k} / a_{r} ; q\right)_{n}}\left(\frac{b_{1} \ldots b_{r}}{a_{1} \ldots a_{r} z}\right)^{n} \\
+\left(\frac{\left(b_{2} / a_{1}, a_{2}, b_{1} / a_{1}, q, a_{1}, a_{4}, \ldots, a_{r}, b_{1} b_{2} / a_{2} a_{3}, b_{3} / a_{3}, \ldots, b_{r} / a_{3}, a_{3} z, q / a_{3} z ; q\right)_{\infty}}{\left(a_{2} / a_{1}, b_{1}, b_{1} b_{2} / a_{1} a_{2}, b_{2}, q / a_{3}, a_{1} / a_{3}, a_{4} / a_{3}, \ldots, a_{r} / a_{3}, b_{3}, \ldots, b_{r}, z, q / z ; q\right)_{\infty}}\right. \\
\times \sum_{k=0}^{\infty} \frac{\left(b_{1} / a_{2}, b_{2} / a_{2}, a_{1} / a_{3} ; q\right)_{k}}{\left(q, a_{1} q / a_{2}, b_{1} b_{2} / a_{2} a_{3} ; q\right)_{k}} q^{k} \\
\times \sum_{n=0}^{\infty} \frac{\left(a_{2} a_{3} q^{1-k} / b_{1} b_{2}, a_{3} q / b_{3}, \ldots, a_{3} q / b_{r} ; q\right)_{n}}{\left(q, a_{3} q^{1-k} / a_{1}, a_{3} q / a_{4}, \ldots, a_{3} q / a_{r} ; q\right)_{n}}\left(\frac{b_{1} \ldots b_{r}}{a_{1} \ldots a_{r} z}\right)^{n} \\
\left.\left.+\mathrm{idem}\left(a_{3} ; a_{4}, \ldots, a_{r}\right)\right)\right]+\mathrm{idem}\left(a_{1} ; a_{2}\right) . \quad(7.9)
\end{gathered}
$$

We have in (7.9) a sum of $2(r-1)$ double sums. Accordingly, for more clarity, let us write the whole expression in 7.9 as

$$
\begin{aligned}
& T_{1}\left(a_{1}, a_{2}\right)+T_{2}\left(a_{1}, a_{2}\right)+\cdots+T_{r-1}\left(a_{1}, a_{2}\right) \\
+ & U_{1}\left(a_{1}, a_{2}\right)+U_{2}\left(a_{1}, a_{2}\right)+\cdots+U_{r-1}\left(a_{1}, a_{2}\right),
\end{aligned}
$$

where, by definition of "idem", $U_{i}\left(a_{1}, a_{2}\right)=T_{i}\left(a_{2}, a_{1}\right)$ for $i=1, \ldots, r-1$. Further, $\sum_{i=2}^{r-1} T_{i}\left(a_{1}, a_{2}\right)=T_{2}\left(a_{1}, a_{2}\right)+\operatorname{idem}\left(a_{3} ; a_{4}, \ldots, a_{r}\right)\left(\right.$ and $\sum_{i=2}^{r-1} U_{i}\left(a_{1}, a_{2}\right)=$ 
$\left.U_{2}\left(a_{1}, a_{2}\right)+\operatorname{idem}\left(a_{3} ; a_{4}, \ldots, a_{r}\right)\right)$. Below, we will selectively perform manipulations with the respective terms $T_{i}$ and $U_{i}(i=1, \ldots, r-1)$.

To evaluate $T_{1}\left(a_{1}, a_{2}\right)$ (and hence also $U_{1}\left(a_{1}, a_{2}\right)$ ), we first shift the index $n$ of the inner sum in $T_{1}\left(a_{1}, a_{2}\right)$ (or, equivalently, in the first term in $\left.(7.9)\right)$ by $-k$ and then interchange the double sum. Symbolically, we apply the interchange of summations as in (5.5). Thus, we obtain (using some elementary identities for $q$-shifted factorials)

$$
\begin{aligned}
T_{1}\left(a_{1}, a_{2}\right)= & \frac{\left(q, a_{2}, \ldots, a_{r}, b_{1} / a_{1}, \ldots, b_{r} / a_{1}, a_{1} z, q / a_{1} z ; q\right)_{\infty}}{\left(q / a_{1}, a_{2} / a_{1}, \ldots, a_{r} / a_{1}, b_{1}, \ldots, b_{r}, z, q / z ; q\right)_{\infty}} \\
& \times \sum_{n=0}^{\infty} \frac{\left(a_{1} a_{2} q / b_{1} b_{2}, a_{1} q / b_{3}, \ldots, a_{1} q / b_{r} ; q\right)_{n}}{\left(q, a_{1} q / a_{3}, \ldots, a_{1} q / a_{r} ; q\right)_{n}}\left(\frac{b_{1} \ldots b_{r}}{a_{1} \ldots a_{r} z}\right)^{n} \\
& \times \sum_{k=0}^{n} \frac{\left(b_{1} / a_{2}, b_{2} / a_{2}, q^{-n} ; q\right)_{k}}{\left(q, a_{1} q / a_{2}, b_{1} b_{2} q^{-n} / a_{1} a_{2} ; q\right)_{k}} q^{k} .
\end{aligned}
$$

Now the inner sum can be evaluated by the terminating $q$-Pfaff-Saalschütz summation (cf. [15, Eq. (II.12)]),

$$
{ }_{3} \phi_{2}\left[\begin{array}{c}
a, b, q^{-n} \\
c, a b q^{1-n} / c
\end{array} ; q, q\right]=\frac{(c / a, c / b ; q)_{n}}{(c, c / a b ; q)_{n}}
$$

which simplifies the last expression, $T_{1}\left(a_{1}, a_{2}\right)$, to

$$
\begin{aligned}
& \frac{\left(q, a_{2}, \ldots, a_{r}, b_{1} / a_{1}, \ldots, b_{r} / a_{1}, a_{1} z, q / a_{1} z ; q\right)_{\infty}}{\left(q / a_{1}, a_{2} / a_{1}, \ldots, a_{r} / a_{1}, b_{1}, \ldots, b_{r}, z, q / z ; q\right)_{\infty}} \\
& \quad \times \sum_{n=0}^{\infty} \frac{\left(a_{1} q / b_{1}, a_{1} q / b_{2}, \ldots, a_{1} q / b_{r} ; q\right)_{n}}{\left(q, a_{1} q / a_{2}, \ldots, a_{1} q / a_{r} ; q\right)_{n}}\left(\frac{b_{1} \ldots b_{r}}{a_{1} \ldots a_{r} z}\right)^{n} .
\end{aligned}
$$

Next, we consider $T_{2}\left(a_{1}, a_{2}\right)$ and $U_{2}\left(a_{1}, a_{2}\right)$. By interchanging the double sums in $T_{2}\left(a_{1}, a_{2}\right)$ and in $U_{2}\left(a_{1}, a_{2}\right)$ we obtain

$$
\begin{aligned}
& T_{2}\left(a_{1}, a_{2}\right)+U_{2}\left(a_{1}, a_{2}\right) \\
& =\frac{\left(b_{2} / a_{1}, a_{2}, b_{1} / a_{1}, q, a_{1}, a_{4}, \ldots, a_{r}, b_{1} b_{2} / a_{2} a_{3}, b_{3} / a_{3}, \ldots, b_{r} / a_{3}, a_{3} z, q / a_{3} z ; q\right)_{\infty}}{\left(a_{2} / a_{1}, b_{1}, b_{1} b_{2} / a_{1} a_{2}, b_{2}, q / a_{3}, a_{1} / a_{3}, a_{4} / a_{3}, \ldots, a_{r} / a_{3}, b_{3}, \ldots, b_{r}, z, q / z ; q\right)_{\infty}} \\
& \quad \times \sum_{n=0}^{\infty} \frac{\left(a_{2} a_{3} q / b_{1} b_{2}, a_{3} q / b_{3}, \ldots, a_{3} q / b_{r} ; q\right)_{n}}{\left(q, a_{3} q / a_{1}, a_{3} q / a_{4}, \ldots, a_{3} q / a_{r} ; q\right)_{n}}\left(\frac{b_{1} \ldots b_{r}}{a_{1} \ldots a_{r} z}\right)^{n} \\
& \quad \times \sum_{k=0}^{\infty} \frac{\left(b_{1} / a_{2}, b_{2} / a_{2}, a_{1} q^{-n} / a_{3} ; q\right)_{k}}{\left(q, a_{1} q / a_{2}, b_{1} b_{2} q^{-n} / a_{2} a_{3} ; q\right)_{k}} q^{k}+\operatorname{idem}\left(a_{1} ; a_{2}\right) .
\end{aligned}
$$

Now, to the inner sum of the first double sum (but not of the second!) in (7.13) we apply the nonterminating ${ }_{3} \phi_{2}$ summation in (7.5), i.e., specifically we apply

$$
\begin{aligned}
\sum_{k=0}^{\infty} \frac{\left(b_{1} / a_{2}, b_{2} / a_{2}, a_{1} q^{-n} / a_{3} ; q\right)_{k}}{\left(q, a_{1} q / a_{2}, b_{1} b_{2} q^{-n} / a_{2} a_{3} ; q\right)_{k}} q^{k} \\
=\frac{\left(a_{2} / a_{1}, b_{2} q^{-n} / a_{3}, b_{1} q^{-n} / a_{3}, b_{1} b_{2} / a_{1} a_{2} ; q\right)_{\infty}}{\left(b_{1} / a_{1}, b_{2} / a_{2}, a_{2} q^{-n} / a_{3}, b_{1} b_{2} q^{-n} / a_{2} a_{3} ; q\right)_{\infty}} \\
-\frac{\left(a_{2} / a_{1}, b_{1} / a_{2}, b_{2} / a_{2}, a_{1} q^{-n} / a_{3}, b_{1} b_{2} q^{-n} / a_{1} a_{3} ; q\right)_{\infty}}{\left(a_{1} / a_{2}, b_{1} / a_{1}, b_{2} / a_{1}, a_{2} q^{-n} / a_{3}, b_{1} b_{2} q^{-n} / a_{2} a_{3} ; q\right)_{\infty}}
\end{aligned}
$$




$$
\times \sum_{k=0}^{\infty} \frac{\left(b_{1} / a_{1}, b_{2} / a_{1}, a_{2} q^{-n} / a_{3} ; q\right)_{k}}{\left(q, a_{2} q / a_{1}, b_{1} b_{2} q^{-n} / a_{1} a_{3} ; q\right)_{k}} q^{k} .
$$

The result of the application of this (two term) summation is that the first term in (7.13), $T_{2}\left(a_{1}, a_{2}\right)$, is split into two parts, one single sum $T_{2}^{\prime}\left(a_{1}, a_{2}\right)$ and one double sum $T_{2}^{\prime \prime}\left(a_{1}, a_{2}\right)$. Formally, we have

$$
T_{2}\left(a_{1}, a_{2}\right)+U_{2}\left(a_{1}, a_{2}\right)=\left[T_{2}^{\prime}\left(a_{1}, a_{2}\right)+T_{2}^{\prime \prime}\left(a_{1}, a_{2}\right)\right]+U_{2}\left(a_{1}, a_{2}\right) .
$$

But $T_{2}^{\prime \prime}\left(a_{1}, a_{2}\right)$ is precisely $-U_{2}\left(a_{1}, a_{2}\right)$ (as can be readily checked), so two terms cancel, thus

$$
T_{2}\left(a_{1}, a_{2}\right)+U_{2}\left(a_{1}, a_{2}\right)=T_{2}^{\prime}\left(a_{1}, a_{2}\right) .
$$

Now, the last expression, $T_{2}^{\prime}\left(a_{1}, a_{2}\right)$, can be simplified to

$$
\begin{aligned}
& \frac{\left(q, a_{1}, a_{2}, a_{4}, \ldots, a_{r}, b_{1} / a_{3}, \ldots, b_{r} / a_{3}, a_{3} z, q / a_{3} z ; q\right)_{\infty}}{\left(q / a_{3}, a_{1} / a_{3}, a_{2} / a_{3}, a_{4} / a_{3}, \ldots, a_{r} / a_{3}, b_{1}, \ldots, b_{r}, z, q / z ; q\right)_{\infty}} \\
& \quad \times \sum_{n=0}^{\infty} \frac{\left(a_{3} q / b_{1}, a_{3} q / b_{2}, \ldots, a_{3} q / b_{r} ; q\right)_{n}}{\left(q, a_{3} q / a_{1}, a_{3} q / a_{2}, a_{3} q / a_{4}, \ldots, a_{3} q / a_{r} ; q\right)_{n}}\left(\frac{b_{1} \ldots b_{r}}{a_{1} \ldots a_{r} z}\right)^{n} .
\end{aligned}
$$

It is easy to see that (7.15) equals (7.12) where $a_{1}$ and $a_{3}$ are interchanged. Collecting all terms, according to (7.10), completes our derivation of Slater's ${ }_{r} \psi_{r}$ transformation formula in (7.1).

We conclude this section considering Slater's ${ }_{r} \psi_{r}$ transformation in its general form:

$$
\begin{array}{r}
{ }_{r} \psi_{r}\left[\begin{array}{l}
a_{1}, a_{2}, \ldots, a_{r} \\
b_{1}, b_{2}, \ldots, b_{r}
\end{array} q, z\right] \\
=\frac{\left(\frac{c_{1}}{a_{1}}, \ldots, \frac{c_{1}}{a_{r}}, c_{2}, \ldots, c_{r}, \frac{q}{c_{2}}, \ldots, \frac{q}{c_{r}}, \frac{b_{1} q}{c_{1}}, \ldots, \frac{b_{r} q}{c_{1}}, A c_{1} z, \frac{q}{A c_{1} z} ; q\right)_{\infty}}{\left(\frac{q}{a_{1}}, \ldots, \frac{q}{a_{r}}, \frac{c_{1}}{c_{2}}, \ldots, \frac{c_{1}}{c_{r}}, \frac{c_{2} q}{c_{1}}, \ldots, \frac{c_{r} q}{c_{1}}, b_{1}, \ldots, b_{r}, A z q, \frac{1}{A z} ; q\right)_{\infty}} \\
\times{ }_{r} \psi_{r}\left[\begin{array}{l}
\frac{a_{1} q}{c_{1}}, \ldots, \frac{a_{r} q}{c_{1}} \\
\left.\frac{b_{1} q}{c_{1}}, \ldots, \frac{b_{r} q}{c_{1}} ; q, z\right]+\operatorname{idem}\left(c_{1} ; c_{2}, \ldots, c_{r}\right),
\end{array}\right.
\end{array}
$$

where $A=a_{1} \ldots a_{r} / c_{1} \ldots c_{r}$, and the series either terminate, or $\left|b_{1} \ldots b_{r} / a_{1} \ldots a_{r}\right|<$ $|z|<1$, for convergence.

The transformation in (7.16) which involves only bilateral series is much more general than the transformation in (7.1) since in (7.16) we have $r$ additional parameters $c_{1}, \ldots, c_{r}$. It is not difficult to see that the special case $c_{i}=a_{i} q, i=1, \ldots, r$, of $(7.16)$ is exactly (7.1). However, for some purposes we rather consider a transformation equivalent to (7.16), see (7.17), below.

The natural question arises whether we can also prove the more general transformation (7.16), which involves only bilateral series, by elementary means. Unfortunately, we were not able to derive (7.16) directly by the method of this article. Instead, we can extend (7.1) to (7.16) by an $r$-fold application of Ismail's argument. This works similar as in Section 6 where we described how the transformation in (6.10) for well-poised ${ }_{2 r} \psi_{2 r}$ series can be extended to the transformation in (6.11). We provide a sketch of how Ismail's argument is applied here.

First, let us transform the identity in (7.16) to an equivalent identity by replacing $z$ by $z / A$, shifting the summation indices by one, and reversing the infinite sums on the right hand side. Specifically, we apply 


$$
\begin{gathered}
\sum_{k=-\infty}^{\infty} \frac{\left(\frac{a_{1} q}{c_{1}}, \ldots, \frac{a_{r} q}{c_{1}} ; q\right)_{k}}{\left(\frac{b_{1} q}{c_{1}}, \ldots, \frac{b_{r} q}{c_{1}} ; q\right)_{k}}\left(\frac{c_{1} \ldots c_{r} z}{a_{1} \ldots a_{r}}\right)^{k} \\
=\sum_{k=-\infty}^{\infty} \frac{\left(\frac{a_{1} q}{c_{1}}, \ldots, \frac{a_{r} q}{c_{1}} ; q\right)_{-1-k}}{\left(\frac{b_{1} q}{c_{1}}, \ldots, \frac{b_{r} q}{c_{1}} ; q\right)_{-1-k}}\left(\frac{c_{1} \ldots c_{r} z}{a_{1} \ldots a_{r}}\right)^{-1-k} \\
=\frac{\left(1-\frac{c_{1}}{b_{1}}\right) \ldots\left(1-\frac{c_{1}}{b_{r}}\right)}{\left(1-\frac{c_{1}}{a_{1}}\right) \ldots\left(1-\frac{c_{1}}{a_{r}}\right)} \frac{b_{1} \ldots b_{r}}{c_{1} \ldots c_{r} z} \sum_{k=-\infty}^{\infty} \frac{\left(\frac{c_{1} q}{b_{1}}, \ldots, \frac{c_{1} q}{b_{r}} ; q\right)_{k}}{\left(\frac{c_{1} q}{a_{1}}, \ldots, \frac{c_{1} q}{a_{r}} ; q\right)_{k}}\left(\frac{b_{1} \ldots b_{r}}{a_{1} \ldots a_{r} z}\right)^{k}
\end{gathered}
$$

to the ${ }_{r} \psi_{r}$ 's on the right hand side of (7.16). Hence, (7.16) becomes

$$
\begin{aligned}
{ }_{r} \psi_{r}\left[\begin{array}{l}
a_{1}, a_{2}, \ldots, a_{r} \\
b_{1}, b_{2}, \ldots, b_{r}
\end{array} ; q, \frac{c_{1} \ldots c_{r} z}{a_{1} \ldots a_{r}}\right] \\
=\frac{\left(\frac{c_{1} q}{a_{1}}, \ldots, \frac{c_{1} q}{a_{r}}, c_{2}, \ldots, c_{r}, \frac{q}{c_{2}}, \ldots, \frac{q}{c_{r}}, \frac{b_{1}}{c_{1}}, \ldots, \frac{b_{r}}{c_{1}}, c_{1} z, \frac{q}{c_{1} z} ; q\right)_{\infty}}{\left(\frac{q}{a_{1}}, \ldots, \frac{q}{a_{r}}, \frac{c_{1} q}{c_{2}}, \ldots, \frac{c_{1} q}{c_{r}}, \frac{c_{2}}{c_{1}}, \ldots, \frac{c_{r}}{c_{1}}, b_{1}, \ldots, b_{r}, z, \frac{q}{z} ; q\right)_{\infty}} \\
\quad \times{ }_{r} \psi_{r}\left[\frac{\frac{c_{1} q}{b_{1}}, \ldots, \frac{c_{1} q}{b_{r}}}{c_{1} q}, \ldots, \frac{c_{1} q}{a_{r}} ; q, \frac{b_{1} \ldots b_{r}}{c_{1} \ldots c_{r} z}\right]+\operatorname{idem}\left(c_{1} ; c_{2}, \ldots, c_{r}\right),
\end{aligned}
$$

where the series either terminate, or $\left|b_{1} \ldots b_{r} / a_{1} \ldots a_{r}\right|<|z|<1$, for convergence.

In (7.17), Slater's general ${ }_{r} \psi_{r}$ transformation is written in a more convenient form for us since here we immediately see that the special case $c_{i}=a_{i}, i=1, \ldots, r$, of (7.17) is exactly (7.1). In the following, we closely follow the final analysis of Section 6 .

It is easy to see that both sides of $(7.17)$ are analytic in each $a_{1}^{-1}, a_{2}^{-1}, \ldots, a_{r}^{-1}$ in a domain around the origin. We know that the identity is true when $a_{i}=c_{i}$, for $i=1, \ldots, r$. What needs to be done is to extend (7.1) first by an additional parameter $a_{1}$, then by $a_{2}$, etc. This means that if we have already extended (7.1) by $a_{1}, \ldots, a_{j}$, what we should have derived is the identity in (7.17) where $a_{i}=c_{i}$, for $i=j+1, \ldots, r$. So, we proceed by induction starting with $j=0$ (identity (7.1)) and ending with $j=r$ (identity (7.17)). In the inductive step, we consider the transformation (7.17) where $a_{i}=c_{i}$, for $i=j+1, \ldots, r$. We call that identity $E_{j+1}$. We need to show that $E_{j+1}$ is true, provided $E_{j}$ is true, which is 7.17 where $a_{i}=c_{i}$, for $i=j, \ldots, r$. We observe that both sides of $E_{j+1}$ are analytic in $1 / a_{j}$ around the origin. The identity is true for $a_{j}=c_{j} q^{-n_{j}}, n_{j}=0,1,2, \ldots$ This follows by the $c_{j} \mapsto c_{j} q^{-n_{j}}$ case of the inductive hypothesis, $E_{j}$. (This can be verified by looking at all the terms involving $j$. Further note that the index of the $j$ th sum is shifted by $-n_{j}$, since the $j$-th bilateral series becomes a unilateral series.) Since $E_{j+1}$ is true for an infinite sequence of $1 / a_{j}$ which has an accumulation point, namely 0 , in the interior of the domain $\mathcal{D}$ of analyticity of $1 / a_{j}$, we can apply the identity theorem to deduce that $E_{j+1}$ is true for $1 / a_{j}$ throughout $\mathcal{D}$. Now, by induction, (7.17) follows, with the general additional parameters $a_{1}, \ldots, a_{r}$.

\section{Transformations of Chu-Gasper-Karlsson-Minton-type}

We say that a basic hypergeometric series is of Gasper-Karlsson-Minton-type if there are $s$ upper parameters $a_{1}, \ldots, a_{s}$ and $s$ lower parameters $b_{1}, \ldots, b_{s}$ such that each $a_{i}$ differs from $b_{i}$ multiplicatively by a nonnegative integer power of $q$, i.e. $a_{i}=b_{i} q^{m_{i}}, m_{i} \geq 0$ (see [15, Sec. 1.9]). Originally, Minton [27] and Karlsson [25] had discovered some corresponding summation formulae for ordinary hypergeometric series (where there are $s$ upper parameters $a_{1}, \ldots, a_{s}$ and $s$ lower parameters 
$b_{1}, \ldots, b_{s}$ such that each $a_{i}$ differs from $b_{i}$ additively by a nonnegative integer, i.e. $\left.a_{i}=b_{i}+m_{i}, m_{i} \geq 0\right)$. Gasper 14 found $q$-analogues of Karlsson and Minton's summations and also extended these to transformation formulae. Later, Chu 10], [1] found bilateral summations and transformations of Gasper-Karlsson-Minton-type which included all the earlier known identities of Gasper-Karlsson-Minton-type as special cases. Accordingly, we call such bilateral identities to be of Chu-GasperKarlsson-Minton-type.

Using here, and in the following, the notation $\left\{x_{\nu}\right\}$ for the $s$ basic hypergeometric parameters $x_{1}, \ldots, x_{s}(\nu=1, \ldots, s)$, and also $|m|=\sum_{i=1}^{s} m_{i}$, for brevity, Chu's [10, Eq. (15)] transformation formula for a specific ${ }_{2+s} \psi_{2+s}$ series of ChuGasper-Karlsson-Minton-type can be stated as follows:

$$
\begin{aligned}
{ }_{2+s} \psi_{2+s}\left[\begin{array}{c}
a, b,\left\{h_{\nu} q^{m_{\nu}}\right\} \\
c, d,\left\{h_{\nu}\right\}
\end{array} ;\right. & \left.\frac{q^{1-N}}{a}\right]=b^{N} \frac{(q, b q / a, c / b, d / b ; q)_{\infty}}{(q / a, q / b, c, d ; q)_{\infty}} \prod_{i=1}^{s} \frac{\left(h_{i} / b ; q\right)_{m_{i}}}{\left(h_{i} ; q\right)_{m_{i}}} \\
& \times{ }_{2+s} \phi_{1+s}\left[\begin{array}{l}
b q / c, b q / d,\left\{b q / h_{\nu}\right\} \\
b q / a,\left\{b q^{\left.1-m_{\nu} / h_{\nu}\right\}} ; q, \frac{c d q^{N-|m|-1}}{b}\right]
\end{array}\right]
\end{aligned}
$$

where $N$ is an arbitrary integer, and where the series either terminate, or $|q / a|<$ $\left|q^{N}\right|<\left|b q^{|m|+1} / c d\right|$, for convergence.

On the other hand, Chu's [11, Theorem 2] summation formula for a specific very-well-poised ${ }_{6+2 s} \psi_{6+2 s}$ series of Chu-Gasper-Karlsson-Minton-type is

$$
\begin{aligned}
6+2 s & \psi_{6+2 s}\left[\begin{array}{c}
q \sqrt{a},-q \sqrt{a}, b, c, d, \frac{a}{d},\left\{h_{\nu}\right\},\left\{\frac{a q^{1+m_{\nu}}}{h_{\nu}}\right\} \\
\sqrt{a},-\sqrt{a}, \frac{a q}{b}, \frac{a q}{c}, \frac{a q}{d}, d q,\left\{\frac{a q}{h_{\nu}}\right\},\left\{h_{\nu} q^{-m_{\nu}}\right\}
\end{array} ;, \frac{a q^{1-|m|}}{b c}\right] \\
= & \frac{(q, q, a q, q / a, a q / b d, a q / c d, d q / b, d q / c ; q)_{\infty}}{(a q / b, a q / c, a q / d, d q / a, q / b, q / c, q / d, d q ; q)_{\infty}} \prod_{i=1}^{s} \frac{\left(a q / d h_{i}, d q / h_{i} ; q\right)_{m_{i}}}{\left(a q / h_{i}, q / h_{i} ; q\right)_{m_{i}}}
\end{aligned}
$$

provided the series either terminates, or $\left|a q^{1-|m|} / b c\right|<1$, for convergence.

Chu established both of these formulae by means of partial fraction expansions. Haglund [17, pp. 415-416] noticed that the transformation in (8.1) can be obtained by specializing Slater's general transformation $(7.16)$ for ${ }_{r} \psi_{r}$ series. It is also true that the summation in (8.2) can be derived by specializing Slater's transformation (6.11) for well-poised ${ }_{2 r} \psi_{2 r}$ series.

Rather then specializing Slater's transformations, in the spirit of this article we give elementary derivations of Chu-Gasper-Karlsson-Minton-type identities. We show here that both of Slater's general transformation formulae, (7.16) and (6.11), can be extended by induction to transformations of Chu-Gasper-Karlsson-Mintontype, see Propositions 8.1 and 8.4 below. The simple analysis involves interchanging of sums, and the expansion of certain factors in terms of the $q$-binomial theorem (8.5) or $q$-Pfaff-Saalschütz summation (7.11), respectively. Finally, we state some interesting special cases (in addition to (8.1) and (8.2)) of the general $\mathrm{Chu}^{-}$ Gasper-Karlsson-Minton-type transformations explicitly, see Corollaries 8.2, 8.3 and 8.6 .

Proposition 8.1 (A general ${ }_{r+s} \psi_{r+s}$ Chu-Gasper-Karlsson-Minton-type transformation). Let $a_{1}, \ldots, a_{r}, b_{1}, \ldots, b_{r}, c_{1}, \ldots, c_{r}, h_{1}, \ldots, h_{s}$, and $z$ be indeterminate, let $m_{1}, \ldots, m_{s}$ be nonnegative integers, let $A=a_{1} \ldots a_{r} / c_{1} \ldots c_{r},|m|=\sum_{i=1}^{s} m_{i}$, and suppose that the series in (8.3) are well-defined. Then 


$$
\begin{aligned}
{ }_{r+s} \psi_{r+s}\left[\begin{array}{c}
a_{1}, \ldots, a_{r},\left\{h_{\nu} q^{m_{\nu}}\right\} \\
b_{1}, \ldots, b_{r},\left\{h_{\nu}\right\}
\end{array} ;, z\right]=\prod_{i=1}^{s} \frac{\left(\frac{h_{i} q}{c_{1}} ; q\right)_{m_{i}}}{\left(h_{i} ; q\right)_{m_{i}}} \\
\times \frac{\left(\frac{c_{1}}{a_{1}}, \ldots, \frac{c_{1}}{a_{r}}, c_{2}, \ldots, c_{r}, \frac{q}{c_{2}}, \ldots, \frac{q}{c_{r}}, \frac{b_{1} q}{c_{1}}, \ldots, \frac{b_{r} q}{c_{1}}, A c_{1} z, \frac{q}{A c_{1} z} ; q\right)_{\infty}}{\left(\frac{q}{a_{1}}, \ldots, \frac{q}{a_{r}}, \frac{c_{1}}{c_{2}}, \ldots, \frac{c_{1}}{c_{r}}, \frac{c_{2} q}{c_{1}}, \ldots, \frac{c_{r} q}{c_{1}}, b_{1}, \ldots, b_{r}, A z q, \frac{1}{A z} ; q\right)_{\infty}} \\
\quad \times{ }_{r} \psi_{r}\left[\begin{array}{c}
\frac{a_{1} q}{c_{1}}, \ldots, \frac{a_{r} q}{c_{1}},\left\{\frac{h_{\nu} q^{1+m_{\nu}}}{c_{1} q}\right\} \\
\frac{b_{1} q}{c_{1}}, \ldots, \frac{b_{r} q}{c_{1}},\left\{\frac{h_{\nu} q}{c_{1}}\right\}
\end{array} ;, z\right]+\operatorname{idem}\left(c_{1} ; c_{2}, \ldots, c_{r}\right),
\end{aligned}
$$

where the series either terminate, or $\left|b_{1} \ldots b_{r} q^{-|m|} / a_{1} \ldots a_{r}\right|<|z|<1$.

Proof. We proceed by induction on $s$. For $s=0$ the transformation is true by Slater's general transformation (7.16). So, suppose the identity is already shown for $s \mapsto s-1$. Then,

$$
\begin{gathered}
{ }_{r+s} \psi_{r+s}\left[\begin{array}{c}
a_{1}, \ldots, a_{r},\left\{h_{\nu} q^{m_{\nu}}\right\} \\
b_{1}, \ldots, b_{r},\left\{h_{\nu}\right\}
\end{array} ;, z\right] \\
=\sum_{k=-\infty}^{\infty} \frac{\left(a_{1}, \ldots, a_{r} ; q\right)_{k}}{\left(b_{1}, \ldots, b_{r} ; q\right)_{k}} \prod_{i=1}^{s-1} \frac{\left(h_{i} q^{m_{i}} ; q\right)_{k}}{\left(h_{i} ; q\right)_{k}} z^{k} \frac{\left(h_{s} q^{m_{s}} ; q\right)_{k}}{\left(h_{s} ; q\right)_{k}} \\
=\frac{1}{\left(h_{s} ; q\right)_{m_{s}}} \sum_{k=-\infty}^{\infty} \frac{\left(a_{1}, \ldots, a_{r} ; q\right)_{k}}{\left(b_{1}, \ldots, b_{r} ; q\right)_{k}} \prod_{i=1}^{s-1} \frac{\left(h_{i} q^{m_{i}} ; q\right)_{k}}{\left(h_{i} ; q\right)_{k}} z^{k}\left(h_{s} q^{k} ; q\right)_{m_{s}} .
\end{gathered}
$$

Now we expand the last $q$-shifted factorial by the terminating $q$-binomial theorem (cf. [15, Eq. (II.4)]),

$$
{ }_{1} \phi_{0}\left[\begin{array}{c}
q^{-n} \\
-
\end{array} ; q, z\right]=\left(z q^{-n} ; q\right)_{n},
$$

which is just the $a \mapsto q^{-n}$ case of (7.3). That is, we apply

$$
\left(h_{s} q^{k} ; q\right)_{m_{s}}=\sum_{j=0}^{m_{s}} \frac{\left(q^{-m_{s}} ; q\right)_{j}}{(q ; q)_{j}}\left(h_{s} q^{k+m_{s}}\right)^{j}
$$

and obtain for the expression in 8.4

$$
\begin{gathered}
\frac{1}{\left(h_{s} ; q\right)_{m_{s}}} \sum_{k=-\infty}^{\infty} \frac{\left(a_{1}, \ldots, a_{r} ; q\right)_{k}}{\left(b_{1}, \ldots, b_{r} ; q\right)_{k}} \prod_{i=1}^{s-1} \frac{\left(h_{i} q^{m_{i}} ; q\right)_{k}}{\left(h_{i} ; q\right)_{k}} z^{k} \sum_{j=0}^{m_{s}} \frac{\left(q^{-m_{s}} ; q\right)_{j}}{(q ; q)_{j}}\left(h_{s} q^{k+m_{s}}\right)^{j} \\
=\frac{1}{\left(h_{s} ; q\right)_{m_{s}}} \sum_{j=0}^{m_{s}} \frac{\left(q^{-m_{s}} ; q\right)_{j}}{(q ; q)_{j}}\left(h_{s} q^{m_{s}}\right)^{j} \\
\times \sum_{k=-\infty}^{\infty} \frac{\left(a_{1}, \ldots, a_{r} ; q\right)_{k}}{\left(b_{1}, \ldots, b_{r} ; q\right)_{k}} \prod_{i=1}^{s-1} \frac{\left(h_{i} q^{m_{i}} ; q\right)_{k}}{\left(h_{i} ; q\right)_{k}}\left(z q^{j}\right)^{k}
\end{gathered}
$$

Note that $j$ is bounded by $m_{s}$, thus the interchange of summations in (8.6) is justified (provided $\left|b_{1} \ldots b_{r} q^{-|m|} / a_{1} \ldots a_{r}\right|<|z|<1$ ). Now we can apply the inductive hypothesis to the inner sum which gives us

$$
\frac{1}{\left(h_{s} ; q\right)_{m_{s}}} \sum_{j=0}^{m_{s}} \frac{\left(q^{-m_{s}} ; q\right)_{j}}{(q ; q)_{j}}\left(h_{s} q^{m_{s}}\right)^{j} \prod_{i=1}^{s-1} \frac{\left(h_{i} q / c_{1} ; q\right)_{m_{i}}}{\left(h_{i} ; q\right)_{m_{i}}} \frac{\left(c_{1} / a_{1}, \ldots, c_{1} / a_{r} ; q\right)_{\infty}}{\left(q / a_{1}, \ldots, q / a_{r} ; q\right)_{\infty}}
$$




$$
\begin{gathered}
\times \frac{\left(c_{2}, \ldots, c_{r}, q / c_{2}, \ldots, q / c_{r}, b_{1} q / c_{1}, \ldots, b_{r} q / c_{1}, A c_{1} z q^{j}, q^{1-j} / A c_{1} z ; q\right)_{\infty}}{\left(c_{1} / c_{2}, \ldots, c_{1} / c_{r}, c_{2} q / c_{1}, \ldots, c_{r} q / c_{1}, b_{1}, \ldots, b_{r}, A z q^{1+j}, q^{-j} / A z ; q\right)_{\infty}} \\
\times \sum_{k=-\infty}^{\infty} \frac{\left(a_{1} q / c_{1}, \ldots, a_{r} q / c_{1} ; q\right)_{k}}{\left(b_{1} q / c_{1}, \ldots, b_{r} q / c_{1} ; q\right)_{k}} \prod_{i=1}^{s-1} \frac{\left(h_{i} q^{1+m_{i}} / c_{1} ; q\right)_{k}}{\left(h_{i} q / c_{1} ; q\right)_{k}}\left(z q^{j}\right)^{k} \\
+\operatorname{idem}\left(c_{1} ; c_{2}, \ldots, c_{r}\right) .
\end{gathered}
$$

In this expression, we again interchange summations, and obtain

$$
\begin{gathered}
\frac{\left(c_{1} / a_{1}, \ldots, c_{1} / a_{r}, c_{2}, \ldots, c_{r}, q / c_{2}, \ldots, q / c_{r}, b_{1} q / c_{1}, \ldots, b_{r} q / c_{1}, A c_{1} z, q / A c_{1} z ; q\right)_{\infty}}{\left(q / a_{1}, \ldots, q / a_{r}, c_{1} / c_{2}, \ldots, c_{1} / c_{r}, c_{2} q / c_{1}, \ldots, c_{r} q / c_{1}, b_{1}, \ldots, b_{r}, A z q, 1 / A z ; q\right)_{\infty}} \\
\times \frac{1}{\left(h_{s} ; q\right)_{m_{s}}} \prod_{i=1}^{s-1} \frac{\left(h_{i} q / c_{1} ; q\right)_{m_{i}}}{\left(h_{i} ; q\right)_{m_{i}}} \sum_{k=-\infty}^{\infty} \frac{\left(a_{1} q / c_{1}, \ldots, a_{r} q / c_{1} ; q\right)_{k}}{\left(b_{1} q / c_{1}, \ldots, b_{r} q / c_{1} ; q\right)_{k}} \prod_{i=1}^{s-1} \frac{\left(h_{i} q^{1+m_{i}} / c_{1} ; q\right)_{k}}{\left(h_{i} q / c_{1} ; q\right)_{k}} z^{k} \\
\times \sum_{j=0}^{m_{s}} \frac{\left(q^{-m_{s}} ; q\right)_{j}}{(q ; q)_{j}}\left(\frac{h_{s} q^{1+k+m_{s}}}{c_{1}}\right)^{j}+\operatorname{idem}\left(c_{1} ; c_{2}, \ldots, c_{r}\right) .
\end{gathered}
$$

We simplify the inner sums, according to 8.5 ,

$$
\sum_{j=0}^{m_{s}} \frac{\left(q^{-m_{s}} ; q\right)_{j}}{(q ; q)_{j}}\left(\frac{h_{s} q^{1+k+m_{s}}}{c_{1}}\right)^{j}=\left(h_{s} q^{1+k} / c_{1} ; q\right)_{m_{s}},
$$

and eventually deduce the proposition.

If, in Proposition 8.1, we set $r=2, a_{1} \mapsto a, a_{2} \mapsto b, b_{1} \mapsto c, b_{2} \mapsto d, c_{1} \mapsto$ $e$, and $z \mapsto e q^{-N} / a b$, then the second term on the right side of (8.3) vanishes. Furthermore, the parameter $c_{2}$ cancels out in the first term on the right side. We obtain

Corollary 8.2. Let $a, b, c, d$, e, and $h_{1}, \ldots, h_{s}$ be indeterminate, let $N$ be an arbitrary integer, $m_{1}, \ldots, m_{s}$ be nonnegative integers, and suppose that the series in (8.7) are well-defined. Then

$$
\begin{aligned}
{ }_{2+s} \psi_{2+s} & {\left[\begin{array}{c}
a, b,\left\{h_{\nu} q^{m_{\nu}}\right\} \\
c, d,\left\{h_{\nu}\right\}
\end{array} ; q, \frac{e q^{-N}}{a b}\right]=\left(\frac{e}{q}\right)^{N} \frac{(e / a, e / b, c q / e, d q / e ; q)_{\infty}}{(q / a, q / b, c, d ; q)_{\infty}} } \\
& \times \prod_{i=1}^{s} \frac{\left(h_{i} q / e ; q\right)_{m_{i}}}{\left(h_{i} ; q\right)_{m_{i}}}{ }_{2+s} \psi_{2+s}\left[\begin{array}{c}
a q / e, b q / e,\left\{h_{\nu} q^{\left.1+m_{\nu} / e\right\}}\right. \\
c q / e, d q / e,\left\{h_{\nu} q / e\right\}
\end{array} ; q, \frac{e q^{-N}}{a b}\right]
\end{aligned}
$$

where the series either terminate, or $|e / a b|<\left|q^{N}\right|<\left|e q^{|m|} / c d\right|$, for convergence.

For $e=d$ the ${ }_{2+s} \psi_{2+s}$ on the right side of 8.7 ) reduces to a ${ }_{2+s} \phi_{1+s}$ series. This gives a transformation for a $2+s \psi_{2+s}$ into a (multiple of a) ${ }_{2+s} \phi_{1+s}$ series which is different from Chu's transformation in (8.1).

We can also reverse the ${ }_{2+s} \psi_{2+s}$ series on the right side of (8.7). We obtain

$$
\begin{aligned}
2_{2+s} \psi_{2+s} & {\left[\begin{array}{c}
a, b,\left\{h_{\nu} q^{m_{\nu}}\right\} \\
c, d,\left\{h_{\nu}\right\}
\end{array} ; q, \frac{e q^{-N}}{a b}\right]=\left(\frac{e}{q}\right)^{N} \frac{(e / a, e / b, c q / e, d q / e ; q)_{\infty}}{(q / a, q / b, c, d ; q)_{\infty}} } \\
& \times \prod_{i=1}^{s} \frac{\left(h_{i} q / e ; q\right)_{m_{i}}}{\left(h_{i} ; q\right)_{m_{i}}}{ }_{2+s} \psi_{2+s}\left[\begin{array}{c}
e / c, e / d,\left\{e / h_{\nu}\right\} \\
e / a, e / b,\left\{e q^{-m_{\nu}} / h_{\nu}\right\}
\end{array} ; q, \frac{c d q^{N-|m|}}{e}\right]
\end{aligned}
$$


where the series either terminate, or $|e / a b|<\left|q^{N}\right|<\left|e q^{|m|} / c d\right|$, for convergence. We immediately see that the special case $e=b q$ of (8.8) is exactly Chu's transformation 8.1.).

Another noteworthy specialization of Proposition 8.1 is simply the $r=1$ case, rewritten in the following corollary:

Corollary 8.3. Let $a, b, c, z$, and $h_{1}, \ldots, h_{s}$ be indeterminate, let $m_{1}, \ldots, m_{s}$ be nonnegative integers, and suppose that the series in (8.9) are well-defined. Then

$$
\begin{aligned}
{ }_{1+s} \psi_{1+s}\left[\begin{array}{c}
a,\left\{h_{\nu} q^{m_{\nu}}\right\} \\
b,\left\{h_{\nu}\right\}
\end{array}\right. & ; q, z]=\frac{(c / a, b q / c, a z, q / a z ; q)_{\infty}}{(q / a, b, a z q / c, c / a z ; q)_{\infty}} \\
& \times \prod_{i=1}^{s} \frac{\left(h_{i} q / c ; q\right)_{m_{i}}}{\left(h_{i} ; q\right)_{m_{i}}}{ }_{1+s} \psi_{1+s}\left[\begin{array}{c}
a q / c,\left\{h_{\nu} q^{1+m_{\nu}} / c\right\} \\
b q / c,\left\{h_{\nu} q / c\right\}
\end{array} ; q, z\right]
\end{aligned}
$$

where the series either terminate, or $\left|b q^{-|m|} / a\right|<|z|<1$, for convergence.

We can set $c=b$ or $c=a q$ in (8.9) to reduce the ${ }_{1+s} \psi_{1+s}$ series on the right side to a ${ }_{1+s} \phi_{s}$ series if we want. If we first let $c \mapsto b$, and then $b \mapsto a q$ in (8.9), the series on the right side of (8.9) reduces to 1 and we would obtain the following summation:

$$
{ }_{1+s} \psi_{1+s}\left[\begin{array}{c}
a,\left\{h_{\nu} q^{m_{\nu}}\right\} \\
a q,\left\{h_{\nu}\right\}
\end{array} ; q, z\right]=\frac{(q, q, a z, q / a z ; q)_{\infty}}{(a q, q / a, z, q / z ; q)_{\infty}} \prod_{i=1}^{s} \frac{\left(h_{i} q / a ; q\right)_{m_{i}}}{\left(h_{i} ; q\right)_{m_{i}}}
$$

where the series either terminates, or $\left|q^{1-|m|}\right|<|z|<1$, for convergence. Now, in the beginning of this article we assumed $|q|<1$. It follows from the convergence condition $\left(\left|q^{1-|m|}\right|<1\right)$ above that $|m|$ needs to be either 0 (thus there are no parameters $h_{i}$ and we are left with a plain ${ }_{1} \psi_{1}$ ), or that the series terminates, and in our case, this would mean that the series reduces just to one term. On the other hand, we may consider $|q|>1$ for a nonempty region of convergence. In this case the convergence condition for the bilateral series on the left side of (8.10) is $1<|z|<\left|q^{1-|m|}\right|$. But unfortunately, our derivation of the identity is not valid in this case. Clearly, if $|q|>1$, we could not have used induction because the inductive basis would already have been false. In total, (8.10) does not give a new summation.

Nevertheless, we can still specialize Corollary 8.3 to a summation by choosing $c \mapsto b$, and then $b \mapsto a q^{1+n}$ where $n$ is a small positive integer. In this case, the series on the right side of (8.9) has only a finite number of terms and can be summed explicitly. For instance, (when $s=n=m=1$ ) we have

$$
{ }_{2} \psi_{2}\left[\begin{array}{c}
a, h q \\
a q^{2}, h
\end{array} ; q, z\right]=\frac{(1-h / a q)-(1-h / a) z / q}{(1-h)} \frac{\left(q^{2}, q, a z, q / a z ; q\right)_{\infty}}{\left(q / a, a q^{2}, z / q, q^{2} / z ; q\right)_{\infty}},
$$

provided $|q|<|z|<1$. For $z=-h / a$ the right side of (8.11) factors (completely into linear factors), and we have

$$
{ }_{2} \psi_{2}\left[\begin{array}{c}
a, h q \\
a q^{2}, h
\end{array} ; q,-\frac{h}{a}\right]=\frac{\left(1-h^{2} / a^{2} q\right)}{(1-h)} \frac{\left(q^{2}, q,-h,-q / h ; q\right)_{\infty}}{\left(q / a, a q^{2},-h / a q,-a q^{2} / h ; q\right)_{\infty}},
$$

provided $|q|<|h / a|<1$.

Let us now consider transformations of Chu-Gasper-Karlsson-Minton-type for well-poised basic series. We have 
Proposition 8.4 (A well-poised ${ }_{2 r+2 s} \psi_{2 r+2 s}$ Chu-Gasper-Karlsson-Minton type transformation). Let $a, a_{1}, \ldots, a_{r}, b_{1}, \ldots, b_{r}$, and $h_{1}, \ldots, h_{s}$ be indeterminate, let $m_{1}, \ldots, m_{s}$ be nonnegative integers, $|m|=\sum_{i=1}^{s} m_{i}$, and suppose that the series in (8.13) are welldefined. Then

$$
\begin{aligned}
& 2 r+2 s=\psi_{2 r+2 s}\left[\begin{array}{c}
b_{1}, \ldots, b_{2 r},\left\{h_{\nu}\right\},\left\{\frac{a q^{1+m_{\nu}}}{h_{\nu}}\right\} \\
\frac{a q}{b_{1}}, \ldots, \frac{a q}{b_{2 r}},\left\{\frac{a q}{h_{\nu}}\right\},\left\{h_{\nu} q^{-m_{\nu}}\right\}
\end{array} ; q,-\frac{a^{r} q^{r-|m|}}{b_{1} \ldots b_{2 r}}\right] \\
& =\frac{\left(a, \frac{q}{a}, a_{2}, \ldots, a_{r}, \frac{q}{a_{2}}, \ldots, \frac{q}{a_{r}}, \frac{a_{2}}{a}, \ldots, \frac{a_{r}}{a}, \frac{a q}{a_{2}}, \ldots, \frac{a q}{a_{r}}, \frac{a_{1} q}{b_{1}}, \ldots, \frac{a_{1} q}{b_{2 r}} ; q\right)_{\infty}}{\left(\frac{q}{b_{1}}, \ldots, \frac{q}{b_{2 r}}, \frac{a q}{b_{1}}, \ldots, \frac{a q}{b_{2 r}}, \frac{a_{2}}{a_{1}}, \ldots, \frac{a_{r}}{a_{1}}, \frac{a_{1} q}{a_{2}}, \ldots, \frac{a_{1} q}{a_{r}}, \frac{a_{1} a_{2}}{a}, \ldots, \frac{a_{1} a_{r}}{a} ; q\right)_{\infty}} \\
& \times \frac{\left(\frac{a q}{a_{1} b_{1}}, \ldots, \frac{a q}{a_{1} b_{2 r}}, \frac{a_{1}}{\sqrt{a}},-\frac{a_{1}}{\sqrt{a}}, \frac{\sqrt{a} q}{a_{1}},-\frac{\sqrt{a} q}{a_{1}} ; q\right)_{\infty}}{\left(\frac{a q}{a_{1} a_{2}}, \ldots, \frac{a q}{a_{1} a_{r}}, \frac{a_{1}^{2}}{a}, \frac{a q}{a_{1}^{2}}, \frac{q}{\sqrt{a}},-\frac{q}{\sqrt{a}}, \sqrt{a},-\sqrt{a} ; q\right)_{\infty}} \prod_{i=1}^{\left(\frac{a_{1} q}{h_{i}}, \frac{a q}{a_{1} h_{i}} ; q\right)_{m_{i}}} \frac{a q}{\left(\frac{a q}{h_{i}}, \frac{q}{h_{i}} ; q\right)_{m_{i}}} \\
& \times{ }_{2 r+2 s} \psi_{2 r+2 s}\left[\begin{array}{l}
\frac{a_{1} b_{1}}{a}, \ldots, \frac{a_{1} b_{2 r}}{a},\left\{\frac{a_{1} h_{\nu}}{a}\right\},\left\{\frac{a_{1} q^{1+m_{\nu}}}{h_{\nu}}\right\} \\
\frac{a_{1} q}{b_{1}}, \ldots, \frac{a_{1} q}{b_{2 r}},\left\{\frac{a_{1} q}{h_{\nu}}\right\},\left\{\frac{a_{1} h_{\nu} q^{-m_{\nu}}}{a}\right\}
\end{array} ; q,-\frac{a^{r} q^{r-|m|}}{b_{1} \ldots b_{2 r}}\right] \\
& +\operatorname{idem}\left(a_{1} ; a_{2}, \ldots, a_{r}\right),
\end{aligned}
$$

where the series either terminate, or $\left|a^{r} q^{r-|m|} / b_{1} \ldots b_{2 r}\right|<1$, for convergence.

Proof. We proceed by induction on $s$. For $s=0$ the transformation is true by Slater's transformation (6.11) for well-poised series. So, suppose the identity is already shown for $s \mapsto s-1$. Then,

$$
\begin{gathered}
2 r+2 s \psi_{2 r+2 s}\left[\begin{array}{c}
b_{1}, \ldots, b_{2 r},\left\{h_{\nu}\right\},\left\{\frac{a q^{1+m_{\nu}}}{h_{\nu}}\right\} \\
\frac{a q}{b_{1}}, \ldots, \frac{a q}{b_{2 r}},\left\{\frac{a q}{h_{\nu}}\right\},\left\{h_{\nu} q^{-m_{\nu}}\right\}
\end{array} ;,-\frac{a^{r} q^{r-|m|}}{b_{1} \ldots b_{2 r}}\right] \\
=\sum_{k=-\infty}^{\infty} \frac{\left(b_{1}, \ldots, b_{2 r} ; q\right)_{k}}{\left(a q / b_{1}, \ldots, a q / b_{2 r} ; q\right)_{k}} \prod_{i=1}^{s-1} \frac{\left(h_{i}, a q^{1+m_{i}} / h_{i} ; q\right)_{k}}{\left(a q / h_{i}, h_{i} q^{-m_{i}} ; q\right)_{k}}\left(-\frac{a^{r} q^{r-\left(m_{1}+\cdots+m_{s}\right)}}{b_{1} \ldots b_{2 r}}\right)^{k} \\
\times \frac{\left(h_{s}, a q^{1+m_{s}} / h_{s} ; q\right)_{k}}{\left(a q / h_{s}, h_{s} q^{-m_{s}} ; q\right)_{k}}=\frac{\left(b_{2 r} q / h_{s}, a q / b_{2 r} h_{s} ; q\right)_{m_{s}}}{\left(a q / h_{s}, q / h_{s} ; q\right)_{m_{s}}} \\
\times \sum_{k=-\infty}^{\infty} \frac{\left(b_{1}, \ldots, b_{2 r} ; q\right)_{k}}{\left(a q / b_{1}, \ldots, a q / b_{2 r} ; q\right)_{k}} \prod_{i=1}^{s-1} \frac{\left(h_{i}, a q^{1+m_{i}} / h_{i} ; q\right)_{k}}{\left(a q / h_{i}, h_{i} q^{-m_{i}} ; q\right)_{k}}\left(-\frac{a^{r} q^{r-\left(m_{1}+\cdots+m_{s-1}\right)}}{b_{1} \ldots b_{2 r}}\right)^{k} \\
\times \frac{\left(a q^{1+k} / h_{s}, q^{1-k} / h_{s} ; q\right)_{m_{s}}}{\left(b_{2 r} q / h_{s}, a q / b_{2 r} h_{s} ; q\right)_{m_{s}}} .
\end{gathered}
$$

Now we expand the last quotient of $q$-shifted factorials by the terminating $q$-PfaffSaalschütz summation (7.11) and obtain for the expression in (8.14)

$$
\begin{gathered}
\frac{\left(b_{2 r} q / h_{s}, a q / b_{2 r} h_{s} ; q\right)_{m_{s}}}{\left(a q / h_{s}, q / h_{s} ; q\right)_{m_{s}}} \sum_{k=-\infty}^{\infty} \frac{\left(b_{1}, \ldots, b_{2 r} ; q\right)_{k}}{\left(a q / b_{1}, \ldots, a q / b_{2 r} ; q\right)_{k}} \prod_{i=1}^{s-1} \frac{\left(h_{i}, a q^{1+m_{i}} / h_{i} ; q\right)_{k}}{\left(a q / h_{i}, h_{i} q^{-m_{i}} ; q\right)_{k}} \\
\quad \times\left(-\frac{a^{r} q^{r-\left(m_{1}+\cdots+m_{s-1}\right)}}{b_{1} \ldots b_{2 r}}\right)^{k} \sum_{j=0}^{m_{s}} \frac{\left(b_{2 r} q^{-k} / a, b_{2 r} q^{k}, q^{-m_{s}} ; q\right)_{j}}{\left(q, b_{2 r} q / h_{s}, b_{2 r} h_{s} q^{-m_{s}} / a ; q\right)_{j}} q^{j} \\
=\frac{\left(b_{2 r} q / h_{s}, a q / b_{2 r} h_{s} ; q\right)_{m_{s}}}{\left(a q / h_{s}, q / h_{s} ; q\right)_{m_{s}}} \sum_{j=0}^{m_{s}} \frac{\left(b_{2 r} / a, b_{2 r}, q^{-m_{s}} ; q\right)_{j}}{\left(q, b_{2 r} q / h_{s}, b_{2 r} h_{s} q^{-m_{s}} / a ; q\right)_{j}} q^{j}
\end{gathered}
$$




$$
\begin{aligned}
& \times \sum_{k=-\infty}^{\infty} \frac{\left(b_{1}, \ldots, b_{2 r-1}, b_{2 r} q^{j} ; q\right)_{k}}{\left(a q / b_{1}, \ldots, a q / b_{2 r-1}, a q^{1-j} / b_{2 r} ; q\right)_{k}} \\
& \times \prod_{i=1}^{s-1} \frac{\left(h_{i}, a q^{1+m_{i}} / h_{i} ; q\right)_{k}}{\left(a q / h_{i}, h_{i} q^{-m_{i}} ; q\right)_{k}}\left(-\frac{a^{r} q^{r-\left(m_{1}+\cdots+m_{s-1}\right)-j}}{b_{1} \ldots b_{2 r}}\right)^{k} .
\end{aligned}
$$

Note that $j$ is bounded by $m_{s}$, thus the interchange of summations in (8.15) is justified (provided $\left|a^{r} q^{r-|m|} / b_{1} \ldots b_{2 r}\right|<1$ ). Now we can apply the inductive hypothesis to the inner sum which gives us

$$
\begin{aligned}
& \frac{\left(b_{2 r} q / h_{s}, a q / b_{2 r} h_{s} ; q\right)_{m_{s}}}{\left(a q / h_{s}, q / h_{s} ; q\right)_{m_{s}}} \sum_{j=0}^{m_{s}} \frac{\left(b_{2 r} / a, b_{2 r}, q^{-m_{s}} ; q\right)_{j}}{\left(q, b_{2 r} q / h_{s}, b_{2 r} h_{s} q^{-m_{s}} / a ; q\right)_{j}} q^{j} \\
& \times \frac{\left(a, q / a, a_{2}, \ldots, a_{r}, q / a_{2}, \ldots, q / a_{r} ; q\right)_{\infty}}{\left(q / b_{1}, \ldots, q / b_{2 r-1}, q^{1-j} / b_{2 r}, a q / b_{1}, \ldots, a q / b_{2 r-1}, a q^{1-j} / b_{2 r} ; q\right)_{\infty}} \\
& \times \frac{\left(a_{2} / a, \ldots, a_{r} / a, a q / a_{2}, \ldots, a q / a_{r}, a_{1} q / b_{1}, \ldots, a_{1} q / b_{2 r-1}, a_{1} q^{1-j} / b_{2 r} ; q\right)_{\infty}}{\left(a_{2} / a_{1}, \ldots, a_{r} / a_{1}, a_{1} q / a_{2}, \ldots, a_{1} q / a_{r}, a_{1} a_{2} / a, \ldots, a_{1} a_{r} / a ; q\right)_{\infty}} \\
& \times \frac{\left(a q / a_{1} b_{1}, \ldots, a q / a_{1} b_{2 r-1}, a q^{1-j} / a_{1} b_{2 r}, a_{1} / \sqrt{a},-a_{1} / \sqrt{a}, \sqrt{a} q / a_{1},-\sqrt{a} q / a_{1} ; q\right)_{\infty}}{\left(a q / a_{1} a_{2}, \ldots, a q / a_{1} a_{r}, a_{1}^{2} / a, a q / a_{1}^{2}, q / \sqrt{a},-q / \sqrt{a}, \sqrt{a},-\sqrt{a} ; q\right)_{\infty}} \\
& \times \prod_{i=1}^{s-1} \frac{\left(a_{1} q / h_{i}, a q / a_{1} h_{i} ; q\right)_{m_{i}}}{\left(a q / h_{i}, q / h_{i} ; q\right)_{m_{i}}} \sum_{k=-\infty}^{\infty} \frac{\left(a_{1} b_{1} / a, \ldots, a_{1} b_{2 r-1} / a, a_{1} b_{2 r} q^{j} / a ; q\right)_{k}}{\left(a_{1} q / b_{1}, \ldots, a_{1} q / b_{2 r-1}, a_{1} q^{1-j} / b_{2 r} ; q\right)_{k}} \\
& \times \prod_{i=1}^{s-1} \frac{\left(a_{1} h_{i} / a, a_{1} q^{1+m_{i}} / h_{i} ; q\right)_{k}}{\left(a_{1} q / h_{i}, a_{1} h_{i} q^{-m_{i}} / a ; q\right)_{k}}\left(-\frac{a^{r} q^{r-\left(m_{1}+\cdots+m_{s-1}\right)-j}}{b_{1} \ldots b_{2 r}}\right)^{k}+\operatorname{idem}\left(a_{1} ; a_{2}, \ldots, a_{r}\right) .
\end{aligned}
$$

In this expression, we again interchange summations, and obtain

$$
\begin{aligned}
& \frac{\left(b_{2 r} q / h_{s}, a q / b_{2 r} h_{s} ; q\right)_{m_{s}}}{\left(a q / h_{s}, q / h_{s} ; q\right)_{m_{s}}} \frac{\left(a, q / a, a_{2}, \ldots, a_{r}, q / a_{2}, \ldots, q / a_{r} ; q\right)_{\infty}}{\left(q / b_{1}, \ldots, q / b_{2 r}, a q / b_{1}, \ldots, a q / b_{2 r} ; q\right)_{\infty}} \\
& \quad \times \frac{\left(a_{2} / a, \ldots, a_{r} / a, a q / a_{2}, \ldots, a q / a_{r}, a_{1} q / b_{1}, \ldots, a_{1} q / b_{2 r} ; q\right)_{\infty}}{\left(a_{2} / a_{1}, \ldots, a_{r} / a_{1}, a_{1} q / a_{2}, \ldots, a_{1} q / a_{r}, a_{1} a_{2} / a, \ldots, a_{1} a_{r} / a ; q\right)_{\infty}} \\
& \times \frac{\left(a q / a_{1} b_{1}, \ldots, a q / a_{1} b_{2 r}, a_{1} / \sqrt{a},-a_{1} / \sqrt{a}, \sqrt{a} q / a_{1},-\sqrt{a} q / a_{1} ; q\right)_{\infty}}{\left(a q / a_{1} a_{2}, \ldots, a q / a_{1} a_{r}, a_{1}^{2} / a, a q / a_{1}^{2}, q / \sqrt{a},-q / \sqrt{a}, \sqrt{a},-\sqrt{a} ; q\right)_{\infty}} \\
& \quad \times \prod_{i=1}^{s-1} \frac{\left(a_{1} q / h_{i}, a q / a_{1} h_{i} ; q\right)_{m_{i}}}{\left(a q / h_{i}, q / h_{i} ; q\right)_{m_{i}}} \sum_{k=-\infty}^{\infty} \frac{\left(a_{1} b_{1} / a, \ldots, a_{1} b_{2 r} / a ; q\right)_{k}}{\left(a_{1} q / b_{1}, \ldots, a_{1} q / b_{2 r} ; q\right)_{k}} \\
& \quad \times \prod_{i=1}^{s-1} \frac{\left(a_{1} h_{i} / a, a_{1} q^{1+m_{i}} / h_{i} ; q\right)_{k}}{\left(a_{1} q / h_{i}, a_{1} h_{i} q^{\left.-m_{i} / a ; q\right)_{k}}\right.}\left(-\frac{a^{r} q^{r-\left(m_{1}+\cdots+m_{s-1}\right)}}{b_{1} \ldots b_{2 r}}\right)^{k} \\
& \quad \times \sum_{j=0}^{m_{s}} \frac{\left(b_{2 r} q^{-k} / a_{1}, a_{1} b_{2 r} q^{k} / a, q^{-m_{s}} ; q\right)_{j}}{\left(q, b_{2 r} q / h_{s}, b_{2 r} h_{s} q^{-m_{s}} / a ; q\right)_{j}} q^{j}+\operatorname{idem}\left(a_{1} ; a_{2}, \ldots, a_{r}\right) .
\end{aligned}
$$

We simplify the last inner sum, according to (7.11),

$$
\sum_{j=0}^{m_{s}} \frac{\left(b_{2 r} q^{-k} / a_{1}, a_{1} b_{2 r} q^{k} / a, q^{-m_{s}} ; q\right)_{j}}{\left(q, b_{2 r} q / h_{s}, b_{2 r} h_{s} q^{-m_{s}} / a ; q\right)_{j}} q^{j}=\frac{\left(a_{1} q^{1+k} / h_{s}, a q^{1-k} / a_{1} h_{s} ; q\right)_{m_{s}}}{\left(b_{2 r} q / h_{s}, a q / b_{2 r} h_{s} ; q\right)_{m_{s}}}
$$

and eventually deduce the proposition. 
It is worth noting the case $a_{1}=b_{1}=-a_{2}=-b_{2}=q \sqrt{a}$ of Proposition 8.4 where the series are specialized to be very-well-poised. Then the first two terms on the right side of (8.13) vanish and we end up with the following $(r-1)$-term transformation:

Proposition 8.5 (A very-well-poised ${ }_{2 r+2 s} \psi_{2 r+2 s}$ Chu-Gasper-Karlsson-Minton type transformation). Let $a, a_{3}, \ldots, a_{r}, b_{3}, \ldots, b_{r}$, and $h_{1}, \ldots, h_{s}$ be indeterminate, let $m_{1}, \ldots, m_{s}$ be nonnegative integers, $|m|=\sum_{i=1}^{s} m_{i}$, and suppose that the series in (8.16) are welldefined. Then

$$
\begin{aligned}
& { }_{2 r} \psi_{2 r}\left[\begin{array}{c}
q \sqrt{a},-q \sqrt{a}, b_{3}, \ldots, b_{2 r},\left\{h_{\nu}\right\},\left\{\frac{a q^{1+m_{\nu}}}{h_{\nu}}\right\} \\
\sqrt{a},-\sqrt{a}, \frac{a q}{b_{3}}, \ldots, \frac{a q}{b_{2 r}},\left\{\frac{a q}{h_{\nu}}\right\},\left\{h_{\nu} q^{-m_{\nu}}\right\}
\end{array} ; q, \frac{a^{r-1} q^{r-2-|m|}}{b_{3} \ldots b_{2 r}}\right] \\
& =\frac{\left(a, \frac{q}{a}, a_{4}, \ldots, a_{r}, \frac{q}{a_{4}}, \ldots, \frac{q}{a_{r}}, \frac{a_{4}}{a}, \ldots, \frac{a_{r}}{a}, \frac{a q}{a_{4}}, \ldots, \frac{a q}{a_{r}} ; q\right)_{\infty}}{\left(\frac{q}{b_{3}}, \ldots, \frac{q}{b_{2 r}}, \frac{a q}{b_{3}}, \ldots, \frac{a q}{b_{2 r}}, \frac{a_{4}}{a_{3}}, \ldots, \frac{a_{r}}{a_{3}}, \frac{a_{3} q}{a_{4}}, \ldots, \frac{a_{3} q}{a_{r}} ; q\right)_{\infty}} \\
& \times \frac{\left(\frac{a_{3} q}{b_{3}}, \ldots, \frac{a_{3} q}{b_{2 r}}, \frac{a q}{a_{3} b_{3}}, \ldots, \frac{a q}{a_{3} b_{2 r}} ; q\right)_{\infty}}{\left(\frac{a_{3} a_{4}}{a}, \ldots, \frac{a_{3} a_{r}}{a}, \frac{a q}{a_{3} a_{4}}, \ldots, \frac{a q}{a_{3} a_{r}}, \frac{a_{3}^{2} q}{a}, \frac{a q}{a_{3}^{2}} ; q\right)_{\infty}} \prod_{i=1}^{s} \frac{\left(\frac{a_{3} q}{h_{i}}, \frac{a q}{a_{3} h_{i}} ; q\right)_{m_{i}}}{\left(\frac{a q}{h_{i}}, \frac{q}{h_{i}} ; q\right)_{m_{i}}} \\
& \times{ }_{2 r} \psi_{2 r}\left[\begin{array}{c}
\frac{q a_{3}}{\sqrt{a}},-\frac{q a_{3}}{\sqrt{a}}, \frac{a_{3} b_{3}}{a}, \ldots, \frac{a_{3} b_{2 r}}{a},\left\{\frac{a_{3} h_{\nu}}{a}\right\},\left\{\frac{a_{3} q^{1+m_{\nu}}}{h_{\nu}}\right\} \\
\frac{a_{3}}{\sqrt{a}},-\frac{a_{3}}{\sqrt{a}}, \frac{a_{3} q}{b_{3}}, \ldots, \frac{a_{3} q}{b_{2 r}},\left\{\frac{a_{3} q}{h_{\nu}}\right\},\left\{\frac{a_{3} h_{\nu} q^{-m_{\nu}}}{a}\right\}
\end{array} ; q, \frac{a^{r-1} q^{r-2-|m|}}{b_{3} \ldots b_{2 r}}\right] \\
& +\operatorname{idem}\left(a_{3} ; a_{4}, \ldots, a_{r}\right),
\end{aligned}
$$

where the series either terminate, or $\left|a^{r-1} q^{r-2-|m|} / b_{1} \ldots b_{2 r}\right|<1$, for convergence.

Finally, let us conclude this section stating an important special case of Proposition 8.5 explicitly. We take $r=3, a_{3} \mapsto f, b_{3} \mapsto b, b_{4} \mapsto c, b_{5} \mapsto d, b_{6} \mapsto e$, and obtain:

Corollary 8.6. Let $a b, c, d, e, f, h_{1}, \ldots, h_{s}$ be indeterminate, let $m_{1}, \ldots, m_{s}$ be nonnegative integers, $|m|=\sum_{i=1}^{s} m_{i}$, and suppose that the series in (8.17) are well-defined. Then

$$
\begin{aligned}
& { }_{6+2 s} \psi_{6+2 s}\left[\begin{array}{c}
q \sqrt{a},-q \sqrt{a}, b, c, d, e,\left\{h_{\nu}\right\},\left\{\frac{a q^{1+m_{\nu}}}{h_{\nu}}\right\} \\
\sqrt{a},-\sqrt{a}, \frac{a q}{b}, \frac{a q}{c}, \frac{a q}{d}, \frac{a q}{e},\left\{\frac{a q}{h_{\nu}}\right\},\left\{h_{\nu} q^{-m_{\nu}}\right\}
\end{array} ; q, \frac{a^{2} q^{1-|m|}}{b c d e}\right]
\end{aligned}
$$

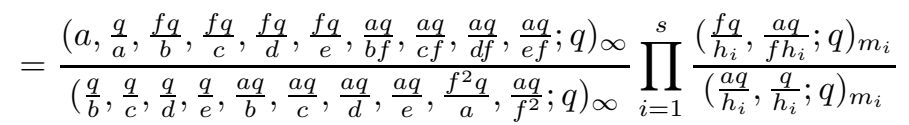

$$
\begin{aligned}
& \times 6+2 s \psi_{6+2 s}\left[\begin{array}{l}
\frac{q f}{\sqrt{a}},-\frac{q f}{\sqrt{a}}, \frac{b f}{a}, \frac{c f}{a}, \frac{d f}{a}, \frac{e f}{a},\left\{\frac{f h_{\nu}}{a}\right\},\left\{\frac{f q^{1+m_{\nu}}}{h_{\nu}}\right\} \\
\frac{f}{\sqrt{a}},-\frac{f}{\sqrt{a}}, \frac{f q}{b}, \frac{f q}{c}, \frac{f q}{d}, \frac{f q}{e},\left\{\frac{f q}{h_{\nu}}\right\},\left\{\frac{f h_{\nu} q^{-m_{\nu}}}{a}\right\}
\end{array} ; q, \frac{a^{2} q^{1-|m|}}{b c d e}\right],
\end{aligned}
$$

where the series either terminate, or $\left|a^{2} q^{1-|m|} / b c d e\right|<1$, for convergence.

The special case $f \mapsto d, e \mapsto a / d$ of (8.17) is Chu's summation in (8.2).

For multidimensional extensions of Corollaries 8.2, 8.3 and 8.6 see [31, where transformations of Chu-Gasper-Karlsson-Minton-type are derived for multiple basic hypergeometric series associated to the root system $A_{n}$.

\section{REFERENCES}

[1] G. E. Andrews, "Applications of basic hypergeometric functions", SIAM Rev. 16 (1974), 441-484. 
[2] G. E. Andrews, q-Series: Their development and application in analysis, number theory, combinatorics, physics and computer algebra, CBMS Regional Conference Lectures Series 66 (Amer. Math. Soc., Providence, RI, 1986).

[3] G. E. Andrews, private communication, June 2000.

[4] R. Askey, "The very well poised ${ }_{6} \psi_{6}$. II", Proc. Amer. Math. Soc. 90 (1984), 575-579.

[5] R. Askey and M. E. H. Ismail, "The very well poised $6 \psi_{6}$ ", Proc. Amer. Math. Soc. 77 (1979), 218-222.

[6] W. N. Bailey, "An identity involving Heine's basic hypergeometric series", J. London Math. Soc. 4 (1929), 254-257.

[7] W. N. Bailey, "Series of hypergeometric type which are infinite in both directions", Quart. J. Math. (Oxford) 7 (1936), 105-115.

[8] W. N. Bailey, "On the basic bilateral basic hypergeometric series ${ }_{2} \psi_{2}$ ", Quart. J. Math. (Oxford) (2) 1 (1950), 194-198.

[9] A.-L. Cauchy, "Mémoire sur les fonctions dont plusieurs valeurs ... ", C.R. Acad. Sci. Paris 17 (1843), 523; reprinted in Oeuvres de Cauchy, Ser. 1 8, Gauthier-Villars, Paris (1893), $42-50$.

[10] W. C. Chu, "Partial fractions and bilateral summations", J. Math. Phys. 35 (1994), 20362042; erratum: ibid 36 (1995), 5198-5199.

[11] W. C. Chu, "Partial fraction expansions and well-poised bilateral series", Acta Sci. Math. (Szeged) 64 (1998), 495-513.

[12] J. F. van Diejen, "On certain multiple Bailey, Rogers and Dougall type summation formulas", Publ. Res. Inst. Math. Sci., Ser. A 33 (1997), 483-508.

[13] J. Dougall, "On Vandermonde's theorem and some more general expansions", Proc. Edinburgh Math. Soc. 25 (1907), 114-132.

[14] G. Gasper, "Summation formulas for basic hypergeometric series", SIAM J. Math. Anal. 12 (1981), 196-200.

[15] G. Gasper and M. Rahman, Basic hypergeometric series, Encyclopedia of Mathematics And Its Applications 35, Cambridge University Press, Cambridge (1990).

[16] R. A. Gustafson, "The Macdonald identities for affine root systems of classical type and hypergeometric series very well-poised on semi-simple Lie algebras", in Ramanujan International Symposium on Analysis (Dec. 26th to 28th, 1987, Pune, India), N. K. Thakare (ed.) (1989), 187-224.

[17] J. Haglund, "Rook theory and hypergeometric series", Adv. Appl. Math. 17 (1996), 408-459.

[18] G. H. Hardy, Ramanujan, Cambridge University Press, Cambridge (1940), reprinted by Chelsea, New York, 1978.

[19] E. Heine, "Untersuchungen über die Reihe ...", J. reine angew. Math. 34 (1847), 285-328.

[20] M. E. H. Ismail, "A simple proof of Ramanujan's ${ }_{1} \psi_{1}$ sum", Proc. Amer. Math. Soc. 63 (1977), 185-186.

[21] F. H. Jackson, "Summation of $q$-hypergeometric series", Messenger of Math. 57 (1921), 101112.

[22] M. Jackson, "On well-poised bilateral hypergeometric series of type ${ }_{8} \psi_{8}$ ", Quart. J. Math. (Oxford) (2) 1 (1950), 63-68.

[23] M. Jackson, "On Lerch's transcendant and the basic bilateral hypergeometric series ${ }_{2} \psi_{2}$ ", $J$. London Math. Soc. 25 (1950), 189-196.

[24] T. H. Koornwinder, "On Zeilberger's algorithm and its $q$-analogue", J. Comp. and Appl. Math. 48 (1993), 91-111.

[25] P. W. Karlsson, "Hypergeometric functions with integral parameter differences", J. Math. Phys. 12 (1971), 270-271.

[26] S. C. Milne, "Balanced ${ }_{3} \phi_{2}$ summation theorems for $U(n)$ basic hypergeometric series", Adv. in Math. 131 (1997), 93-187.

[27] B. M. Minton, "Generalized hypergeometric function of unit argument", J. Math. Phys. 11 (1970), 1375-1376.

[28] R. J. Rogers, "Third memoir on the expansion of certain infinite products", Proc. London Math. Soc. 26 (1894), 15-32.

[29] M. Schlosser, "Summation theorems for multidimensional basic hypergeometric series by determinant evaluations", Discrete Math. 210 (2000), 151-169.

[30] M. Schlosser, "A simple proof of Bailey's very-well-poised $6 \psi_{6}$ summation", Proc. Amer. Math. Soc., to appear. 
[31] M. Schlosser, "Multilateral transformations of Chu-Gasper-Karlsson-Minton-type", in preparation.

[32] D. B. Sears, "Transformations of basic hypergeometric functions of any order", Proc. London Math. Soc. (2) 53 (1951), 181-191.

[33] L. J. Slater, "General transformations of bilateral series", Quart. J. Math. (Oxford) (2) 3 (1952), 73-80.

[34] L. J. Slater, "Integrals representing general hypergeometric transformations", Quart. J. Math. (Oxford) (2) 3 (1952), 207-216.

[35] L. J. Slater, Generalized hypergeometric functions, Cambridge Univ. Press, London/New York, 1966

[36] L. J. Slater and A. Lakin, "Two proofs of the ${ }_{6} \psi_{6}$ summation theorem", Proc. Edinburgh Math. Soc. (2) 9 (1953-57), 116-121.

[37] G. N. Watson, "The continuations of functions defined by generalized hypergeometric series", Trans. Cambridge Phil. Soc. 21 (1909), 281-299.

Department of Mathematics, The Ohio State University, 231 West 18th Avenue, Columbus, Ohio 43210, USA

E-mail address: mschloss@math.ohio-state.edu 